\title{
Opportunities and challenges for polymer science using synchrotron-based infrared spectroscopy
}

\author{
Gary J. Ellis ${ }^{1}$ and Michael C. Martin ${ }^{2}$ \\ ${ }^{1}$ CSIC, Institute of Polymer Science \& Technology (ICTP), c/ Juan de la Cierva 3, 28006 Madrid, Spain. \\ gary.ellis@csic.es, \\ ${ }^{2}$ Advanced Light Source Division, Lawrence Berkeley National Laboratory, Berkeley, California, USA. \\ MCMartin@lbl.gov
}

\begin{abstract}
Many breakthrough advances that are often at the cutting edge of technological development take place at synchrotron facilities. In the case of IR spectroscopy, the last decade has seen important developments in infrared instrumentation incorporated to and developed at synchrotron facilities to take advantages of the characteristics of the bright synchrotron source. In this feature article we describe the origin and nature of synchrotron infrared microspectroscopy, highlighting some of the key developments that will make a future impact in the study of macromolecular materials, and illustrating several applications in the area of polymer science.
\end{abstract}

\section{Keywords}

Synchrotron infrared microspectroscopy; polymer materials; FTIR; nano spectroscopy; imaging

\section{Introduction}

Infrared (IR) spectroscopy is a high information content molecular characterization technique that is well known and widely utilized in all areas of polymer science. Of its main virtues, firstly, it has been accessible to scientists for over a century, and a deep knowledge base on the information it provides is available. Secondly, it boasts to be one of the most versatile analytical techniques available with regard to sampling methodologies. This can allow the study of materials in almost any form, shape or size and in a wide range of environments, including extreme conditions. Thirdly, it can be relatively easily coupled to other analytical methods. Infrared microspectroscopy (IRMS) is a particularly successful example of such a coupled or "hyphenated" technique. Interest in IRMS arose fundamentally from two desires: the possibility to obtain IR spectra from very small samples, and the possibility to spatially resolve very small regions on a sample. Although IRMS was first developed in the 1950's [1] it was not until about 30 years later, with the technological advances in Fourier transform IR hardware and computational data processing methods, that it became accessible. With subsequent developments and improvements in instrument hardware and software, it has evolved into a powerful characterization tool for chemical and/or structural mapping or imaging of materials that has been very successfully exploited in many areas of biological and materials sciences, including a wide range of applications in the study of polymeric materials. For more information the reader is referred to several interesting sources $\left[{ }^{2}\right]$.

For microscopic analysis of polymeric materials many techniques are available to study different types of structures over a variety of size scales, ranging from macroscopic to nanometric scales, and these are broadly illustrated in Figure 1 in terms of the structural sizes 
observable by the respective techniques with respect to lateral spatial resolution. Thus, another important aspect of IR spectroscopy is its strong complimentary with other techniques. Traditionally, IR spectroscopy spans the region between $13,000-10 \mathrm{~cm}^{-1}$ (near IR: $0.77-2.5 \mu \mathrm{m}$, mid IR: $2.5-25 \mu \mathrm{m}$ and far IR: $25-1000 \mu \mathrm{m}$ ). Albeit access to the study of many structures in polymeric materials is fundamentally limited by the wavelength of the radiation, recent developments in instrumentation are providing new opportunities to obtain chemical and structural information from important structural elements in polymers. Amongst these developments synchrotron infrared microspectroscopy (SIRMS) in the mid IR spectral region has experienced important advances over the last decades.

Figure 1. Different (microscopic) techniques for the analysis of polymeric materials. The filled boxes represent the normal size range for each technique, whilst the dotted lines show the most advanced ranges available. The red area represents the size range accessible to study by infrared methods highlighting the strong complementarity with other characterisation techniques.

A commercial IR microscope was first coupled to the output from a synchrotron storage ring in the USA in the early 1990's [ $\left.{ }^{3}\right]$, and it was shown that, thanks to the brightness superiority of the source, the synchrotron IR signal can be 3 orders of magnitude higher than conventional incandescent IR (also called globar) sources [ $\left.{ }^{4}\right]$. This, combined with the relatively low cost of construction compared to other synchrotron-based techniques, has meant that the technique has grown rapidly to become an essential feature at synchrotron facilities throughout the world, and currently $>25$ IR beamlines are available to users, many of these dedicated to IR microspectroscopy (see Table 1, Annex). As a consequence there has been a steady growth in studies using SIRMS in many scientific areas, as can be appreciated from Figure 2 where the evolution of published work up to 2015 using SIRMS is presented along with an overview of the distribution in the different areas where it is making an impact. Many of the most exciting developments have been in, and are driven by, the biological and biomedical sciences and consequently life sciences accounts for almost $60 \%$, by far the largest output in terms of scientific publications.

Figure 2. The growing impact of SIRMS. Published works using SIRMS up until 2015, and an indication of the broad scientific areas where the technique is being applied, a breakdown from the last 250 papers. Source: SCOPUS and Web of Science.

Whilst the number of examples of applications in polymeric materials is still relatively modest at around $6 \%$ of the published work, many opportunities are available to take advantage of the new developments in a growing number of IR techniques available at synchrotron facilities, along with several challenges to adapt methodologies effectively exploited for studies in other disciplines to help resolve key problems in polymer materials science. Some of the developments that we consider most relevant for this area are described herein.

\section{Why use a synchrotron for infrared spectroscopy?}


The crucial parameter for why synchrotrons make appealing infrared sources is the effective size of the electron source for the light, which is a combination of the small physical size of the electron beam and the small emittance angle into which the light is emitted. In modern storage rings it is generally of the order of a few microns or smaller, and can be very effectively coupled into an infrared microscope. A schematic arrangement for SIRMS is presented in Figure 3a. As in conventional IR microspectroscopy, reflective (mirror) optics are employed, the only difference being the replacement of the internal thermal source for the IR beam from a storage ring. Essentially the experimental procedure is identical, Figure $3 \mathrm{~b}$ : The visible image of the microscope is used to select an area of interest on the sample to be studied, either in transmission (shown) or reflection mode. A remote aperture delimits the smallest point of interest, and the IR radiation collected through the aperture is passed to the detector to generate an IR spectrum. In a confocal arrangement, a second aperture is employed to also define the illumination. By raster scanning the sample using a motorised microscope stage, a matrix of spectra can be obtained with a spatial contrast that is, in the first instance, conditioned by the size of the aperture(s). Subsequently, the analysis of differential spectral details can then be used to provide the distribution of chemical or structural variations in the sample.

Figure 3. (a) Schematic of SIRMS, adapted and reproduced from Ref. [5], (b) IR microspectroscopic mapping schematic 1 = Positioning and localization of area of interest and visible image obtained of sample (in this case a polymorphic film of isotactic polypropylene), 2 = Selection of region of interest, definition of aperture size and raster scanning the motorised microscope stage to obtain a spectrum at each grid point, and $4=$ Identifying spectral differences and generating maps (or images) from these, and (c) Graph of signal to noise ratio vs. aperture size for thermal and synchrotron IR sources, recorded at ALS, LBNL.

The ability to spatially resolve chemical or structural features of a material under study is a fundamental aspect of the technique and naturally as the aperture size is reduced the signal decreases as the spatial resolution increases until the energy-limited signal-to-noise ratio becomes too low to practically use; with conventional IR sources this practical limit is generally around $20 \mu \mathrm{m}$. Here the use of a synchrotron IR source provides an enormous advantage. Synchrotron storage rings provide broadband radiation that is almost linear over the whole IR region with extraordinarily high brightness when compared to a conventional globar source due to the small physical size of the electron beam and the low divergence of the emitted light beam. Due to these special characteristics of infrared synchrotron radiation, described in detail elsewhere $\left[{ }^{6}\right]$, when projected through the microscope the synchrotron IR beam produces a small spot of between $2-10 \mu \mathrm{m}$ at the sample in the mid-IR. Thus, when the energy performance is compared to a conventional thermal IR source the advantage for high spatial resolution can be a factor of 1000 , Figure $3 \mathrm{c}$. It is important to emphasise that this significant energy advantage is due to the brightness or spectral brilliance (i.e. number of photons per unit area per unit solid angle) and not raw power, thus no adverse heating effects of the sample are observed $\left.{ }^{7}\right]$. Using synchrotron radiation high quality IR spectra can be routinely obtained through even very small apertures $<3 \mu \mathrm{m}\left[{ }^{8}\right]$. 
At this juncture it is important to make several points regarding spatial resolution. The resolution limit $\Delta r$, or the minimum resolvable separation of two points on a sample is not fundamentally aperture limited, but rather is limited by the diffraction of the IR radiation, as expressed by the Rayleigh criterion:

$$
\Delta r=\frac{0.61 \lambda}{n \sin \theta}
$$

where $\lambda$ is the wavelength of the radiation, $n$ is the refractive index of the medium and $\theta$ is the acceptance angle of the microscope objective, where $n \sin \theta$ is commonly known as the numerical aperture, NA of the optical system. In the case of IR microscopes, depending on the magnification of the optics, the Cassegrain (or Schwartschild) reflective objectives employed typically have NA values between $0.4-0.7$. Thus, if we wish to study, for example, the distribution in a sample of a carbonyl containing species with a $\mathrm{C}=\mathrm{O}$ stretching vibration at around $1715 \mathrm{~cm}^{-1}(\lambda=5.8 \mu \mathrm{m})$, using a microscope with a $32 x$ objective with a typical $N A=0.6$, then it can be seen from Eq. 1 that $\Delta r$ will approximately correspond to the wavelength of the band under study. An important consequence is that spectral features that have bands at longer wavelengths will not be spatially resolved at the aperture size even if this is smaller. This is very nicely illustrated by comparing the spatial contrast obtained from a lithographed polymer pattern on an IR transparent substrate that was mapped through a $3 \times 3 \mu \mathrm{m}^{2}$ aperture with $2 \mu \mathrm{m}$ steps, Figure 4 . The effect of diffraction is clearly observed by comparing the clearly resolved IR image generated from a C-H stretching band at around $2940 \mathrm{~cm}^{-1}$ (aprox. $3.4 \mu \mathrm{m}$ wavelength) to the image generated from the $\mathrm{C}=\mathrm{O}$ stretching band at around $1715 \mathrm{~cm}^{-1}$ (approx. $5.8 \mu \mathrm{m}$ wavelength), where the blurring of details in the pattern arises from diffraction. This also illustrates a practical point. When monitoring spectral features at longer wavelengths, matching the aperture size to the wavelength of interest is recommended from the point of view of optimising the signal-to-noise ratio, as smaller apertures restrict the amount of IR light available. On the other hand, at shorter wavelengths with a tightly focused synchrotron beam, the smallest aperture limit is probably around $2 \mu \mathrm{m}$, matching closely the region of characteristic high frequency stretching vibrations observed in many polymeric materials $(-\mathrm{OH},-\mathrm{NH},-\mathrm{CH})$.

Figure 4. Effect of wavelength on spatial resolution in synchrotron IR microspectroscopy. See text for details. Visible image of a lithographically patterned polymer on a $\mathrm{CaF}_{2}$ substrate, and spectrum observed from the position indicated compared to the substrate spectrum. IR images generated from integration of the bands at $3.4 \mu \mathrm{m}$ and $5.8 \mu \mathrm{m}$. Polymer sample and images kindly provided by Mark Tobin (Australian Synchrotron) and Paul Dumas (Synchrotron SOLEIL).

Inevitably, in order to study details of many types of domains in polymers, the highest spatial resolution is needed. Although with a synchrotron IR source, diffraction limited IR spectroscopy is routine, this is often not enough. Several methods are available to improve the spatial resolution. Firstly, Carr et al. $\left[{ }^{9}\right]$ demonstrated that by using confocal microscope apertures, diffraction effects can be significantly reduced, and under this arrangement $\Delta r=\lambda / 2$ can be achieved. Although clearly an advantage for improving spatial resolution, the inevitable consequence is a large reduction of energy throughput, and for this reason a synchrotron source is an essential requirement. Several studies discuss the optimisation of features of IR 
microscopes to achieve diffraction-limited measurements with SR using various strategies, such as spatial oversampling (i.e. microscope step size smaller than aperture size) or Fourier self-deconvolution (FSD) that can be effective in enhancing the fidelity of an image $\left[{ }^{10}\right]$.

\section{Applications of SIRMS in polymer science}

SIRMS is finding applications in the study of a wide range of problems in polymer science that extend from the study of compositional heterogeneity in multicomponent systems to the study of structured materials or dynamic processes. Some examples are highlighted below, with emphasis on the capabilities of the SIRMS technique, and how new developments can push these applications and others forward in the future.

\section{Multicomponent materials}

The ability to discriminate small particles or domains of diverse nature, and map their distributions within polymer matrices has been highlighted in several studies. As an example Katayama, et al. $\left[{ }^{11},{ }^{12}\right]$ combined SIRMS with morphological observations to study the composition distribution in high-impact polypropylene (hiPP) particles produced by reactor blending in a two-stage process using Zeigler-Natta catalysts. By mapping different crosssectioned particles as a function of ethylene feed concentration they discovered a heterogeneous distribution of ethylene, corresponding to the copolymer, which concentrated at the surfaces and voids of the particles, Figure 5 (top). Their observations provided evidence to support a model $\left[{ }^{13}\right]$ proposed for hiPP particle growth. In another study, the spatial distribution of a poly(ethylene glycol)-isocyanate (PEG-iso) crosslinker and different natural waxes in high-amylose content starch-glycerol blends was examined across the thickness of microtomed thin films by transmission SIRMS $\left[{ }^{14}\right]\left[{ }^{15}\right]$. This is very important in the area of sustainable flexible packaging materials where multicomponent strategies to overcome the poor flexibility, inherent brittleness and poor water barrier properties of carbohydrate polymers such as starch require efficient characterization methods. The authors were able to obtain clear correlations between the spatial distribution of the components, the film morphology and hydrophobicity.

Conventional IR microspectroscopy has been successfully employed to study phase distributions and phase separation phenomena in polymer blends $\left[{ }^{2 c}\right]$ using differential chemical signatures from the blend components in order to determine the distribution of each phase. Several SIRMS studies on polymer blends have been reported, including immiscible polymer blends of poly(ethylene terephthalate) with polystyrene, PET/PS and poly(methyl methacrylate), PET/PMMA prepared by solid-state blending $\left[{ }^{16}\right]$, or melt-blended poly(aryl ether ether ketone) / Vectra LCP, PEEK/Vectra $\left[{ }^{17}\right]$. However, in all cases where the blend components could be successfully differentiated, the dispersed phase domain size was larger than the diffraction limit for the characteristic spectral signature, and the characterization of the distribution when the domain size is below this limit becomes difficult. Another frequent limitation arises from strongly overlapping bands that cannot be effectively distinguished, particularly when the blended polymers present a similar chemical composition. In recent study, Russell et al. $\left[{ }^{18}\right]$ used SIRMS to follow phase separation phenomena in two microbial biosynthetic polyhydroxyalkanoate blends; poly(3-hydroxybutyrate) (PHB) and poly(3hydroxyoctanoate) (PHO). These polymers have very similar spectral signatures, since the only 
structural difference between them is due to the length of the side chain in the repeat unit that changes from a methyl group, $-\mathrm{CH}_{3}$ in $\mathrm{PHB}$ to 5 carbon side chain, $-\left(\mathrm{CH}_{2}\right)_{4} \mathrm{CH}_{3}$ in $\mathrm{PHO}$. A particularly interesting aspect of this work was the preparation of deuterated $\mathrm{PHO}$ that provided C-D stretching frequencies far removed from the $\mathrm{CH}$ region allowing very clear discrimination between the two phases as a function of composition, Figure 5 (bottom).

Figure 5. SIRMS studies on heterogeneous polymeric materials. Top. Visible images of hiPP particles and IR images showing the distribution of ethylene with different copolymer compositions: (a) $18 \% \mathrm{E}$, (b) $37 \% \mathrm{E}$ and (c) $60 \% \mathrm{E}$, and (d) the distribution of ethylene around internal voids in a particle with $50 \% \mathrm{E}$. High values on colour scales indicate higher ethylene concentration. Reproduced with permission from Ref. 12 (C) Wiley Periodicals Inc. Bottom. SIRMS study of PHB / dPHO polymer blend cross sections with $50 \%$ (left) and 60\% (right) w/w PHB: (a)(b) optical image of films, (b)(c) IR image of C-H region (2800$3050 \mathrm{~cm}^{-1}$ ) corresponding to PHB, and (d)(e) IR image of C-D region (2000-2250 $\mathrm{cm}^{-1}$ ) corresponding to $\mathrm{dPHO}$, and representative monomer structures for (g) PHB and (h) dPHO. Reprinted (adapted) with permission from R.A. Russell, et al. Biomacromolecules 2014, 15, 644-649. (C) 2014 American Chemical Society.

In the case of structured polymers and polymer composites, several recent examples can be found, such as the analysis of laser microperforated biodegradable poly(hydroxyalkanoate) films as biocompatible substrates for tissue repair strategies $\left[{ }^{19}\right]$. Here, evidence crystallinity changes in laser-ablated poly(3-hydroxybutyrate-co-3-hydroxyvalerate) (PHBHV) films was correlated with cell-growth and migration, suggesting that increased amorphicity at the pore rims could facilitate cell adhesion and promote templated cell growth and migration through the pores. The SIRMS study of structured hierarchical or layered materials has enormous impact in the areas of and cultural heritage materials and forensic science. The ability to elucidate the nature and distribution of multiple components within artworks, although not always polymeric in nature, or contemporary historical materials, is of great significance not only for identification and authentication purposes, but also for the difficult task of conservation. In this respect plastics-based artefacts have relatively short lifetime and complex aging behaviour that depends on their exposure during their lifetime to different thermal and oxidative environments, moisture, chemicals, etc. that can cause important damage. In a recent study, the photo-oxidative decomposition of artificially aged acrylonitrile-butadienestyrene ( $A B S$ ) resins was studied as a model system for the understanding of degradation processes in contemporary historical materials made from ABS dating back to the early 1960s. In this work $\left[{ }^{20}\right]$ the authors obtained high resolution chemical maps from thin microtomed sections in transmission mode with apertures as small as $3 \times 3 \mu m^{2}$. Good s/n ratio was obtained through these small aperture sizes due to the high brightness of the synchrotron source, and high contrast images could be obtained that allowed identification of the different degradation products, revealing their distribution and depth as a function of the exposure time to the aging conditions, Figure 6 (top). Finally, the comparison of the ageing model to contemporary materials provided important information on both the state of preservation and possible formulation variations in contemporary ABS products.

Figure 6. Examples of SIRMS for the study of cultural heritage materials and forensic samples. Top: Study of photodoxidation and additive migration in a thin section of an acrylonitrile-butadiene-styrene 
(ABS) fragment after $1000 \mathrm{~h}$ of photo-oxidative ageing. Images under (a) visible light and (b) UV light; (c) profiles of the chemical maps calculated as the average along the width of the map and normalized by the bulk value. The scale for the CO profile was divided by a factor of 10 . (d-j) IR chemical maps of the section, map size: $210 \times 250 \mu \mathrm{m}^{2}$, beam size and step size: $10 \times 10 \mu \mathrm{m}^{2}$; showing the integrated net intensity of (d) $\mathrm{OH}$ band, (e) CN band, (f) CO band, ( $g$ ) vinyl-butadiene band, (h) styrene band, (i) transbutadiene band and (j) band at $911 \mathrm{~cm}^{-1}$; (k) average FTIR spectra along a horizontal line, at different depths. Reproduced with permission from Saviello, et al., Anal. Chim. Acta 843 (2014) 59-72, (C) Elsevier. Middle: SIRMS characterisation of component migration in automotive paints. (a) Optical micrograph of a paint cross-section obtained from a red Mazda 3, (b) IR chemical image highlighting the distribution of polyurethane $\left(1690 \mathrm{~cm}^{-1}\right)$ melamine $\left(1550 \mathrm{~cm}^{-1}\right)$, and isophthalic alkyd $\left(1236 \mathrm{~cm}^{-1}\right)$, and (e) an RGB image by combining and overlapping the previous images allowing the visualisation of the local distribution of all three components. Reproduced with permission from Maric, et al., Talanta 118 (2014) 156-161, (C) Elsevier. Bottom: Characterisation of a glitter particle (a) Image of the distribution of the ratio of $\mathrm{CH}_{3}$ (at $2990 \mathrm{~cm}^{-1}$ ) to $\mathrm{CH}_{2}$ (at $2960 \mathrm{~cm}^{-1}$ ), superimposed on an optical image of a glitter sample. The colour code represents the value of the ratio. (b) Profiles of the $1780 \mathrm{~cm}^{-1}$ and the $1341 \mathrm{~cm}^{-1}$ bands, as well as the $\mathrm{CH}_{3} / \mathrm{CH}_{2}$ ratio across the glitter cross-section. (c) synchrotron infrared micro-spectra across the 11 layers, colour coded such that similar spectra have the same colour. Reproduced with permission from Vernoud, et al., Forensic Science International 2011, 210, 47-51, (c) Elsevier.

Additive migration is also of fundamental importance in forensic science. Paint chips or smears are amongst the most commonly encountered trace evidence in vehicle-related accidents, and their characterisation is of fundamental interest, and SIRMS can provide a valuable tool, being able to identify different layer composition, and interlayer particle distribution. However, a virtually unconsidered approach is the study of interlayer component migration, which is a temporal process in all paints, and unless understood can make the unequivocal identification of a sample questionable. As an example, Figure 6 (middle) shows the optical and SIRMS images corresponding to the migration of melamine, one of the most commonly employed cross-linking additives, and pigments from the basecoat to the clearcoat in automotive paint samples $\left[{ }^{21},{ }^{22}\right]$. Access to this type of information can be vital for forensic identification. Another illustrative example in forensics is glitter, ubiquitous in everyday life as a form of decoration. It is present in many cosmetics, clothing, inks and paints, and is widely used by both young and old in arts and crafts. In criminal trials, trace (or transfer) evidence may assist in showing an association between the victim/suspect and crime scenes. Because glitter is small and light, it is readily transferred and retained on contact, often without our knowledge. For these reasons, glitter may be regarded as important associative evidence in criminal investigations $\left.{ }^{23}\right]$. The variety of glitter is astounding and it is found in an assortment of colours, shapes, and sizes. Thus, the more ways a glitter particle may be characterised, the smaller the subclass of evidence it will fall into, and the greater its potential value as evidence. Glitter is a multilayer material and most glitter particles are composed of three and sometimes many more distinct layers. Whilst some (one or more) may be metallised (aluminium) layers, the majority of the layers consist of polymers. In addition to the general shape, size, colour, thickness, and specific gravity of a glitter particle, the number of layers and their thickness and composition are important characteristics. Because some of the layers may be quite thin $1<$ $10 \mu \mathrm{m})$, SIRMS becomes a valuable characterisation tool. Measurements of cross sections that determine the number of layers, the thickness of each layer, and the chemistry (infrared spectrum) of each layer can help to place a questioned glitter particle into a smaller subclass of trace evidence (it would be statistically less likely that glitter particles having all these same properties would by chance be found at the crime scene, on the victim, and on the suspect, for 
example). Figure 6 (bottom) illustrates the analysis of such a multi-layered glitter particle using SR-FTIR micro-spectroscopy $\left[{ }^{24}\right]$.

The use of polymeric materials in device applications is widespread, and several studies have already demonstrated that SIRMS can be a powerful technique for the characterization of diverse systems both under static and dynamic conditions. Veder et al.[25] studied the nature, distribution and effects of undesirable water inclusions at the buried interface in polymeric solid-contact ion-selective electrodes (ISE), using SIRMS as an analytical method to evaluate different strategies, such as combinations of hydrophobic ISE (PMMA-PDMA copolymer) with different hydrophobic or hydrophilic solid contact materials such as poly(3-octylthiophene 2,5diyl) (POT) or poly (3,4-ethylenedioxythiophene)/poly(styrenesulfonate) (PEDOT/PSS) to effectively eliminate water inclusions. In another study, Beattie et al. $\left[{ }^{26}\right]$ used a novel attenuated total reflectance (ATR) reflective element to study a confined polyelectrolyte multilayer bio-lubricant film based on chitosan-hyaluronic acid, where they obtained spatially resolved spectra of hydration water that allowed them to describe the nature of hydrogen bonding in pressure contact regions providing some insight on lubrication mechanisms. The morphology and homogeneity of organic-inorganic interfaces in electro-dialysis ion exchange resins for water desalination has been characterized for the first time using SIRMS $\left[{ }^{27}\right]$, providing concise information on the spatial distribution of components in several systems containing diverse polymeric binders via the study of microtomed polished cross-sections using an ATR mapping technique. Other studies on device-based systems include the photodegradation in polymer solar cells $\left[{ }^{28}\right]$, and the electro-optical response of encapsulated nematic liquid crystals (NLC) in electrohydrodynamically-generated polymeric microcavities $\left[{ }^{29}\right]$.

\section{Anisotropic materials}

Molecular chain orientation and its distribution are primordial to the understanding of the properties of many polymeric materials. The IR spectra of anisotropic materials are highly sensitive to the polarization of the incident radiation with respect to the reference direction of the sample under study, and the ability to obtain spatial maps or images representative of the orientation distribution is fundamental to establishing and understanding structure-property correlations. One of the unique features of the synchrotron source is its inherent polarization $\left[{ }^{30}\right]$, the nature of which depends on the geometry of extraction from the storage ring (edge or bending magnet radiation, or both). It has been demonstrated that the polarization properties obtained at the sample stage of a microscope are quasi-linear and virtually independent of the contribution from edge radiation (radially polarized) since the continuous field bending magnet radiation dominates $\left[{ }^{31}\right]$. From the point of view of microsampling, here SIRMS offers a clear advantage over conventional microspectroscopy under many circumstances, for example, where only small samples are available or high spatial contrast is needed, because energy-reducing polarizing optical elements are not required. One of the first examples of the use of the inherent polarization of the synchrotron IR beam was a study of the crystalline orientation in isotactic polypropylene (iPP) $\left.{ }^{17,32,33}\right]$. Here the sensitivity of the average chain orientation within the different polymorphs to a polarized IR beam was demonstrated and exploited to characterize the transcrystalline interphase region of fibre-reinforced iPP composite films. Precise structural and orientational information was correlated closely with morphological evidence and $\mathrm{x}$-ray micro-diffraction, supporting a well-accepted model for the formation of a shear induced cylindritic $\beta$-phase from oriented $\alpha$-phase row-nuclei proposed 
by Varga and Karger-Kocsis $\left[{ }^{34}\right]$. In the aforementioned studies IR dichroism, an established tool for studying orientational properties of materials, was not exploited. IR dichroism is characterised by the dichroic difference $\Delta A=A_{\Perp}-A_{\perp}$ or the dichroic ratio $\mathrm{D}=A_{\Perp} / A_{\perp}$, where $A_{\|}$ and $A_{\perp}$ correspond to the absorbance of the sample of linearly polarised light parallel or perpendicular to the sample axis, respectively, generally obtained by placing a polariser before the sample and rotating it to these two orthogonal positions. Conventional IR mapping sample stages do not facilitate rotation of the area of interest on the sample in order to generate correlated dichroic spectra, and rotation of the plane of polarization of the synchrotron beam was, of course, impractical. However, by combining the natural polarization of the synchrotron IR beam with careful sample positioning using a home-built sample rotator, Santoro et al. $\left[{ }^{31}\right]$ examined local orientation in drawn poly(ethylene terephthalate) (PET). Here the changes in dichroic ratios (D) and chain conformation through the necking zone of a drawn sample were followed. They further examined the orientation and conformational features inside micronsized micro-crazes that were generated perpendicular to the draw direction [ $\left.{ }^{35}\right]$. The crazing phenomenon is common in mechanically strained polymers and is a precursor for fracture and product failure. High-resolution IR microspectroscopy, using small $4 \times 4 \mu \mathrm{m}^{2}$ confocal apertures and oversampling with high precision $(1 \mu \mathrm{m})$ steps to maximize spatial contrast particularly in the high frequency spectral region, combined with Raman microspectroscopy and conformational analysis, provided clear evidence for increased crystallinity in the micro-crazes consistent with a local strain-hardening mechanism, and suggested that different conformational rearrangements occur during crazing.

A promising area for the study of orientation phenomena in polymeric systems, without the need to rotate either the sample or the polarising element, both actions being sources of positional errors, is the combination of SIRMS with polarization modulation (PM). In this technique, a photo-elastic modulator (PEM) is employed to alternate the polarization of the beam between orthogonal states at high frequency and the spectra are acquired using dual channel detection. Information on the use of this technique for polymer studies can be found in the literature $\left[{ }^{36},{ }^{37}\right]$. However, it was not until the PM technique was coupled to a synchrotron IR source at the IRIS beamline at BESSY-II (HZB-Berlin) $\left.{ }^{38}\right]$, using the arrangement shown in Figure 7 that highly spatially resolved PM IR spectra became available. In this experimental setup, the natural polarization axis of the synchrotron IR beam is "purified" along the principal quasi-linear polarization axis using a wire grid polarizer to produce perfectly linear polarized beam, and the PEM is placed directly after the polarizer between the spectrometer and the microscope at an angle of 450 to the optical axis. By operating the PEM at a peak retardation of $\lambda / 2$, two perpendicular linearly polarized orthogonal states are produced at high frequency (typically $100 \mathrm{kHz}$ ), and a synchronous sampling demodulator is employed to obtain two spectra that correspond to the sum $\left(A_{\text {sum }}=A_{\|}+A_{\perp}\right)$ and difference $\left(A_{\text {diff }}\right.$ $\left.=A_{\|}-A_{\perp}\right)$ spectra from the orthogonal states. Thus, a normalized polarization modulated dichroic difference (PMDD) spectrum can be generated from the ratio, $A_{\text {diff }} / A_{\text {sum }}$. Whilst the raw PMDD spectrum can be used directly to characterise the distribution of molecular orientation in the material under study, a true dichroic difference spectrum requires calibration of the abscissa using a thin film polarizer in place of the sample in positions parallel and perpendicular to the polarizer $\left[{ }^{39}\right]$, thus allowing the extraction of orientation functions, habitually employed for the characterization and quantification of the degree of molecular orientation $\left[{ }^{40},{ }^{41}\right]$. A key advantage of the PM technique is that a background spectrum is not required since both signals are simultaneously acquired at each sample position, automatically compensating for atmospheric changes or instrumental fluctuations over time. Finally, high 
spatial resolution can only be obtained using a high-brightness synchrotron source, since the aperture limit using a conventional incandescent source is around $25 \mu \mathrm{m}$ due to the important energy loss though the PEM. With a synchrotron beam, good quality spectra have been successfully recorded through a $4 \times 4 \mu \mathrm{m}^{2}$ aperture.

PM-SIRMS has been employed to study hydrogen bonding and orientation in wood polymer fibres $\left[{ }^{42}\right]$, and chain orientation in several semicrystalline polymers, including isotactic polypropylene (iPP) and poly(1-butene) (i-PBu) $[38,43]$, and becomes a powerful tool for the study of many anisotropic phenomena in diverse polymeric materials.

Figure 7. Left: Schematic experimental setup for PM-SIRMS, and right: (a) absorbance spectrum and (b) PMDD spectrum at different film orientations for a thin film of polypropylene. Reproduced with permission from Schmidt, et al. Infrared Physics \& Technology 49 (2006) 69-73, (c) Elsevier.

The spherulitic morphology of semicrystalline polymers is an area of fundamental interest $\left[{ }^{44}\right]$, and much debate still exists with regard to many issues regarding the formation and nature of these superstructures. As an example of the high sensitivity of the PM-SIRMS technique to polymer chain orientation, Figure 8a presents the PMDD spectra obtained from three positions on an isothermally crystallised large spherulite (shown) in a thin film sample of i-PBu, in transmission mode through $10 \times 10 \mu \mathrm{m}^{2}$ apertures. The spectral differences observed between spectra $A, B$ and $C$ correspond to differences in the average chain orientation in the zones defined by the microscope aperture. It is well accepted that in a chain folded polymer crystal in any plane of spherulitic growth the average molecular chain axis is orthogonal to the crystal growth axis in the radial direction. Thus, in the spectrum obtained from the centre or nucleus of the spherulite, crystal growth occurs in all directions leading to an almost invariant PMDD spectrum. However, at positions B and C the average molecular orientation of the i-PBu chains is clearly defined and is completely orthogonal, as shown by the almost symmetrical inversion of the spectral bands in the corresponding PMDD spectra.

Figure 8. Examples of PM-SIRMS in various polymers (see text for details). (a) PMDD spectra recorded at different positions from an isothermally crystallised i-PBu spherulite; (b) PMDD band intensity of vs. position of spectrum recorded along the lines indicated from $\mathrm{PHB}$ spherulites crystallised at $\mathrm{A} 100 \circ \mathrm{C}$ and $B$ 90ㄷ; (c) Schematic representation of lamellar twisting showing the orientation of the polymer crystal axes; (d) PMDD intensity of a crystalline band of iPP in the $\beta$-phase of a polymorphic interphase, recorded along lines 1 and 2 marked in the POM image; and (e) PMDD intensity of the same band mapped over a similar spectral region. (a), (d) and (e) adapted from Ref. 43.

The origin of the periodic optical ring banding behaviour observed in many polymers and polymer blends is one area of controversy $\left[{ }^{45}\right]$. Traditionally the origin of this phenomenon has been attributed to the Keith and Padden model of periodic spiralling or twisting of the crystalline lamellae during crystallization $\left[{ }^{46}\right]$, and much morphological evidence provided from the correlation of polarised optical microscopy (POM) images with SEM and AFM measurements, and from structural evidence provided by $\mathrm{x}$-ray microdiffraction, the latter being successfully employed in the study of lamellar twisting in many polymers. However, 
recently PM-SIRMS has emerged as a powerful and complementary tool. As an example, Figure $8 \mathrm{~b}$ presents the variations in the PMDD intensity, $I_{P M D D}$ for the $895 \mathrm{~cm}^{-1}$ band of poly(3hydroxybutyrate), PHB, associated to a backbone C-C stretching vibration characteristic of the polymer chain, as a function of the position of spectra recorded along the lines marked $A$ and $B$ on the POM images recorded from thin film samples that were crystallised isothermally from the melt at two different temperatures. The periodic oscillation observed in the intensity of the band arises from differences in the average chain alignment associated with the twisting of the lamellae in the radial direction, see Figure $8 \mathrm{c}$. The highest values of the oscillation coincide with the edge-on arrangement of the polymer crystals when the polymer chains, or the c-axis, lie parallel to the microscope plane. The observed pitch of the oscillation correlates closely with the band spacing seen in the corresponding POM images, and an increase in the twist pitch is observed with increasing crystallization temperature, showing pitch values in close agreement to those observed from earlier $x$-ray microdiffraction studies on $\mathrm{PHB}\left[{ }^{47},{ }^{48},{ }^{49}\right]$. PMSIRMS was also used to characterise lamellar twisting for the first time in the $\beta$-cylindritic phase of transcrystalline fibre-reinforced iPP $\left[{ }^{43}\right]$, Figure 8d. By following the PMDD intensity of the $995 \mathrm{~cm}^{-1}$ crystalline band a large twist pitch was observed in the b-axis of the crystals in the direction orthogonal to the fibre axis, whereas at a distance parallel to the fibre much less variation in PMDD intensity was observed (line 2). By mapping a region on a similar sample, some evidence for progressive cooperativity between the rotating crystal axes formed from neighbouring nuclei on a reinforcing fibre could be inferred, Figure 8e. Whilst earlier SIRMS studies showed that the formation of the $\beta$-cylindritic layer originated in $\alpha$-phase row nuclei $\left[{ }^{32,33}\right]$ agreed with the Varga model $\left[{ }^{34}\right]$, it is clear that further work is needed to fully understand the nature of the developing polymorphic interphase and the influence of, amongst other factors, the population of sheared nuclei and subsequent spatial confinement on the polymorphic crystallization process in these thin film models. Thus PM-SIRMS provides a powerful and complementary method to investigate spherulitic structure, and as improvements in the technique advance, more detailed studies will be forthcoming.

Another particularly relevant technique for the polarization-dependant study of polymeric materials is infrared spectroscopic ellipsometry (IRSE) $\left[{ }^{50}\right]$. The principal of IRSE, Figure 9a, is that when linearly polarised light illuminates a sample surface the light reflected becomes elliptically polarised and by measuring the reflection coefficients $r_{p}$ and $r_{s}$ the ellipsometric parameters $\tan \Psi$ and $\Delta$, which describe the change in the state of polarisation, can be determined. This field is fast growing, and has been extensively developed at BESSY-II (HZB Berlin) using synchrotron radiation $\left[{ }^{51},{ }^{52}\right]$, particularly driven by the interest in the characterisation of functional polymer materials and biosensors in working or sensing environments. Several interesting studies have been reported using synchrotron IRSE on smart functional polymer materials, such as the $\mathrm{pH}$-dependant $\left[{ }^{53,54,55}\right]$ or temperature dependant $\left.{ }^{56}\right]$ swelling behaviour of polyelectrolyte brushes, and more recently the extension of these studies to protein absorption and repulsion properties of nanometre-thin stimuli-responsive smart polymer brushes $\left[{ }^{57}\right]$. The experimental arrangement for this type of studies is presented in Figures $9 \mathrm{~b}$ and $\mathrm{c}$. In the in-situ cell, the IR beam impinges on the sample through a Si substrate to which the polymer brushes are grafted, which acts as the top window of the cell containing an aqueous solution. An example of the $\mathrm{pH}$ dependant switching behaviour of poly(acrylic acid), PAA is presented in Figures $9 c, d$ and e $\left[{ }^{54}\right]$. In Figure $9 c$ the reduction in the band at $1728 \mathrm{~cm}^{-1}$, due to the carboxylic group $(\mathrm{COOH})$ in the differential $\tan \Psi$ spectra, on increasing the $\mathrm{pH}$ from 2 to 10 by adding $\mathrm{K}^{+}$ions, clearly indicated a decrease in the number of protonated groups on the polymer brush. Subsequent proton dissociation evidenced by the 
simultaneous increase in the symmetric and asymmetric COO- stretching bands at 1414 and $1560 \mathrm{~cm}^{-1}$, respectively led to conformational changes in the brushes through swelling and elongation (or stretching) caused by the electrostatic repulsion due to the negatively charged $\mathrm{COO}^{-}$groups, denominated switching. By adding $\mathrm{Cl}^{-}$ions, the switching behaviour was shown to be reversible albeit with some hysteresis, Figure $9 \mathrm{~d}$ and a model for the switching behaviour was proposed, Figure 9e.

Figure 9. Synchrotron infrared spectroscopic ellipsometry. (a) Principle of IRSE measurements, (b) typical in-situ cell showing a polymer brush sample in solution, where the green line indicates incident and reflected radiation from the interface region including the polymer brushes, the red line the reflected radiation from the backside of the sample, (c) differential $\tan \Psi$ spectra, normalised to the spectrum at $\mathrm{pH} \mathrm{2,} \mathrm{recorded} \mathrm{on} \mathrm{increasing} \mathrm{the} \mathrm{pH}$ of the solution to $\mathrm{pH} 10$ by adding $\mathrm{KOH}$, (d) graph of $\tan \Psi$ intensity of $\mathrm{COO}$ - stretch at $1560 \mathrm{~cm}^{-1}$ as a function of $\mathrm{pH}$ (red line increasing $\mathrm{pH}$, blue line decreasing $\mathrm{pH}$ ) compared to the degree of swelling measured with visible in-situ ellipsometry, and (f) model for the switching process showing effects of conformational changes in the brushes as a function of the content of $\mathrm{Cl}^{-}$(green) and $\mathrm{K}^{+}$ions (red). (a) and (c-e) adapted and reproduced with permission from D. Aulich, et al., Langmuir 2010, 26, 12926-12932, (C) American Chemical Society, (b) reproduced with permission from Furchner, et al., Thin Solid Films, 541, 41 (2013), (c) Elsevier.

The brightness of the synchrotron source is an important advantage for this low divergence technique, and has led to an important reduction in the required area of the sample to sub$\mathrm{mm}$ scale with monolayer sensitivity $\left[{ }^{58}\right]$, and a special setup allowing mapping measurements has been developed $\left[{ }^{51},{ }^{59}\right]$. Recently, using polarization-dependent ellipsometric IR microspectroscopy a lateral spatial resolution $<40 \mu \mathrm{m}$ has been demonstrated on thin polyimide films, allowing the study of homogeneity $\left.{ }^{60}\right]$. Further advances towards increased spatial resolution are envisaged in the near future, making this a powerful technique for thickness and anisotropy variations of thin polymeric architectures in dynamic experiments as a function of external stimuli.

\section{Dynamic processes}

One of the most interesting developments has been the study of dynamic processes by SIRMS, due to the ability to obtain high signal-to-noise performance with short acquisition times. A number of dynamic measurements using SIRMS have been previously reported, but these have been mainly limited to the evolution with time of a single point on the sample, with examples in the study of biological systems $\left[{ }^{61}\right]$, catalysis $\left[{ }^{62}\right]$ and gas adsorption [ $\left.{ }^{63}\right]$. Mapping larger areas of materials using the raster scanning approach is generally tempered by the nature of the transient process, as seen previously, since the acquisition times required to generate the maps limit both the temporal and spatial resolution available $\left[{ }^{64}\right]$. However, some examples where the timescale of the mapping process is adequate to study the sample dynamics include the study of the polymorphic transformation in isotactic poly(1-butene), i-PBu $\left[{ }^{65}\right]$, and the study of more complex crystallization phenomena in the same polymer, such as self-nucleation $\left.{ }^{[66}\right]$. When i-PBu is crystallised from the melt phase it forms a thermodynamically unstable tetragonal polymorph (known as Form II) with an $11_{3}$ helical chain conformation that undergoes a solid-state transition to the more stable trigonal (Form I) polymorph with a $3_{1}$ helical conformation, which occurs over a period of several days. The IR spectral signatures of 
the two crystal forms can be clearly distinguished, Figure 10a, and the spatial distribution of the II-I transformation within a single spherulitic structure in a thin film sample, was followed by mapping the $923 / 905 \mathrm{~cm}^{-1}$ band ratios characteristic of Form I and From II, respectively, through $6 \times 6 \mu \mathrm{m}^{2}$ apertures at different times during the transformation process, Figure 10b. It was observed that although the polymorphic transformation is slow, it starts immediately after crystallization, and slight differences observed in the rate of transformation, highlighted by comparing spectra at different times, Figure 10c probably arise from variations in lamellar thickness along the spherulite radius, since it is known that the II/I transformation occurs faster for thicker crystals. ${ }^{[67]}$

Figure 10. SIRMS of polymorphic transition in i-PBu: (a) Characteristic IR spectra of crystalline polymorphs, Form I and Form II; (b) POM image of a single i-PBu spherulite isothermally crystallized at $90 \circ C$ showing $60 \times 60 \mu^{2}$ area mapped through a $10 \times 10 \mu^{2}$ microscope apertures and (c) corresponding spectra obtained after $2.5 \mathrm{~h}$ and $41 \mathrm{~h}$ (at positions 1 and 2 marked on image) and Form II/Form I intensity ratio maps obtained. SIRMS study of self-nucleation in i-PBu as a function of seeding temperature, between 128-134으 (see text for details): (d) POM images showing mapped regions (red boxes marked with dimensions shown below images) with II/I intensity ratio images and HCA images obtained after recrystallization at each seeding temperature; (e) Graph of polymorphic index vs. seeding temperature for $\mathrm{II} / \mathrm{I}$ intensity ratios obtained using univariate and multivariate approaches. (a)-(c) reproduced with permission from Ref. 65, (d) and (e) adapted and reproduced from Ref. 66 with permission from the Royal Society of Chemistry.

In a more recent study ${ }^{[66]}$, the self-nucleation behaviour of i-PBu was studied by SIRMS using a three-step thermal history protocol whereby specially prepared thin-film samples containing isolated Form I i-PBu spherulites were (i) heated at a constant rate to self-nucleation temperatures, $T_{s}$ ranging between $128-134 \stackrel{\circ}{\circ}$ and held there for 5 minutes, (ii) cooled to a recrystallization temperature of $90 \circ \mathrm{C}$ and held for $10 \mathrm{~min}$, time enough to develop a selfnucleated morphology, then (iii) heated to $109^{\circ} \mathrm{C}$ and held there in order to artificially fix the morphology, since that temperature is below the melting temperature of Form II, but too high to allow any significant crystal growth within the timescale of the IR microspectroscopy measurements. The morphology was measured by polarised optical microscopy and IR microspectroscopic mapping during the hold stage in step (iii), and the corresponding POM and IR images (generated from the II/I band intensity ratios, corresponding to the 924 and 905 $\mathrm{cm}^{-1}$ bands) obtained at each $T_{s}$ are presented in Figure 10d. It was seen that at low selfnucleation temperatures, the original Form I spherulite was essentially preserved and seeds the growth of a Form II corona at its periphery, known as cross-nucleation ${ }^{68}$. However, the interior of the spherulites showed a mixed crystal structure of Form II and Form I, the latter increasing with increasing $T_{s}$ until, at higher values, the memory of the Form I structure is completely lost and the sample recrystallizes uniquely in Form II. This is illustrated in Figure 10e where the polymorphic index $\mathrm{II} / \mathrm{I}$ obtained from both univariate and multivariate (Hierarchical Cluster Analysis, HCA) approaches are represented. In the former, the average II/I intensity ratios obtained from spectra from different areas of the crystalline morphology are plotted, whilst in the latter the index was obtained from the average spectra generated from the corresponding spatial regions identified from cluster analysis based on all the spectral variations in the spectral range from $1350-800 \mathrm{~cm}^{-1}$, where several spectral differences between the polymorphs are observed. A close correlation was found between the data obtained. Whilst the results obtained are highly complementary with previous studies on self- 
nucleation in $\mathrm{i}-\mathrm{PBu}^{[69,70]}$, the SIRMS images provide a concise representation of the spatial and structural distribution of the polymorphs, and the high signal-to-noise quality provided by the synchrotron source is beneficial for both time-limited mapping experiments, and for the application of chemometric image analysis methods. HCA methods for the analysis of IR spectroscopic data have been particularly successful in SIRMS studies in the life sciences ${ }^{71}$, but have only recently found application in polymeric materials. It emerges as a powerful tool not only for the study of polymorphism, but also any chemical or structural variation, particularly when there are multiple or overlapping bands in the spectra that are characteristic of the features to be studied.

\section{D and 3D imaging}

Two-dimensional (2D) focal plane array (FPA) detectors are being employed in IR microspectroscopy $\left[{ }^{72,73,74}\right]$ due to their advantages, particularly for the study of dynamic processes such as phase separation or degradation [ $\left.{ }^{75}\right]$. Recently, 2D infrared spectromicroscopic imaging has become significantly faster and is experimenting rapid growth and success when coupled to conventional IR microspectrometers. An FPA detector that is sensitive to infrared light consists of an array of $\mathrm{HgCdTe}$ (or MCT) photovoltaic pixels. The output voltages from each pixel are digitised and Fourier transformed into spectra that are used to construct an image of the analysed sample, usually in the form of a chemical map. The associated image data are represented in the form of a hyperspectral cube of information. Each pixel represents an entirely separate, complete infrared spectrum corresponding to the projected area of the sample. The number of pixels (and therefore spectra) that can be acquired at one time is based on the size of the FPA. The best commercially available FPA detectors today have $128 \times 128$ pixel arrays, and have a readout fast enough to permit rapidscan FTIR acquisition, permitting the simultaneous collection of 16,384 individual spectra. Thus, when compared to single-point detectors, FPAs are incredibly fast at acquiring spectral images. When coupled to an infrared microscope, an FPA detector can be used to measure infrared spectral images of samples with spatial resolutions up to the diffraction-limit, depending on optical magnification, and with the caveat that the FPA precludes using confocal optics for optimal diffraction-limited focus. However, the infrared light transmitted or reflected by the sample is now being shared by all the pixels in the FPA detector, meaning that the signal-to-noise ratio for each spectrum from each pixel is significantly lower compared to the full signal being detected by a larger single-point detector. This loss of signal-to-noise is often worth trading off for the greatly shortened total time to acquire a spectral image, but ways to provide more photons, such as via synchrotron imaging beamlines, are being actively pursued.

A particularly attractive possibility when coupling a synchrotron IR source to an FPA array detector is full-frame time-lapse chemical imaging of dynamic processes. Coupling the small synchrotron beam footprint across a larger array detector is non-trivial and the spatial resolution of a confocal single point detector configuration will still be superior $\left[{ }^{76}\right]$. However, the speed of data acquisition advance still motivated coupling synchrotron IR sources to pixelated detectors, which was first reported in 2006 at the NSLS, Brookhaven [ ${ }^{77}$ ] demonstrating fast imaging with high spatial discrimination corresponding to $0.5 \mu \mathrm{m}$ per pixel using a high magnification (74x) objective. However, this initial approach resulted in poorer spectral quality, and subsequently multiple-beam sample illumination strategies have been developed $\left[{ }^{78}\right]$ to cover a wider area on the sample whilst maintaining the source brightness. 
At the NSLS, a system was developed to couple four synchrotron beams to an FPA that allowed "one-shot" images to be obtained over reasonably sized areas, Figure 11. The feasibility of this approach was demonstrated by Stavitski et al. $\left.{ }^{[9}\right]$ who studied the photodegradation of four small $(5 \mu \mathrm{m})$ polystyrene (PS) beads under UV irradiation. They observed a progressive loss in intensity of an aromatic C-H stretching band between $3050-3000 \mathrm{~cm}^{-1}$ and the appearance of a broad carbonyl band between 1815-1620 $\mathrm{cm}^{-1}$ originating from a mixture of oxygenated species due to carboxylic acids and esters. The spectral changes and FTIR images of the beads generated from the $\mathrm{CH}$ region and carbonyl regions at different irradiation times over a period of $14 \mathrm{~h}$ are shown in Figure 11, and both the spectral changes and kinetics are consistent with polymer chain cleavage and photooxidation $\left[{ }^{80}\right]$. The authors pointed out that even though the photodegradation was studied over many hours, less than two minutes were required for each full frame image with a high SNR, clearly paving the way for spatially resolved studies of faster transient processes, such as polymerization reactions, thermally induced phase separation, crystallization phenomena, etc.

Figure 11. Multiple-beam 2D imaging arrangement and time-lapsed imaging of the photooxidation of polystyrene. (a) imaging setup using 4 beams and (b) light path in the optical matching box showing a single beamlet for clarity, (c) illuminated area of the $128 \times 128$ pixel FPA detector and (d) the enlarged $36 \times 36$ pixel area, equivalent to a $20 \times 20 \mu \mathrm{m}^{2}$ area on the sample showing almost uniform illumination. (e) visible image of four $5 \mu \mathrm{m}$ polystyrene beads. (f) and (g) show the IR images of the beads after distinct UV exposure times, showing (h) a decrease in the aromatic $\mathrm{C}-\mathrm{H}$ stretching band and (i) an increase in the $\mathrm{C}=\mathrm{O}$ stretching vibration. (j) kinetics of the chemical transformations for the $\mathrm{C}-\mathrm{H}$ and $\mathrm{C}=\mathrm{O}$ bands corresponding to the shaded areas in ( $h$ ) and (i). Adapted and reproduced with permission from E. Stavitski, et al., Anal. Chem. 85 (2013) 3599, (C) American Chemical Society.

An extension on this strategy was used in the development of the IRENI beamline at the SRC in Wisconsin, which employed 12 beams to illuminate the focal plane array detector [ $\left.{ }^{81}\right]$. The smaller size storage ring at SRC allowed the collection of an unusually large horizontal swath of synchrotron light, which was split into twelve beamlets. Each of these 12 beams was collimated and then repackaged into a $3 \times 4$ array that entered the FTIR bench and IR microscope, Figure 12. To fully illuminate $128 \times 128$ pixels, an asymmetric optical scheme was used with a $15 \mathrm{x}$ condenser and a $74 \mathrm{x}$ objective to give a projected per-pixel resolution of $0.54 \mathrm{x}$ $0.54 \mu \mathrm{m}^{2}$. Two examples to demonstrate the potential in the study of polymeric materials are presented in the lower panel of Figure 12. The first case shows analysis of a $3 \mu \mathrm{m}$ thick crosssection of a multilayer oxygen barrier film that was prepared by embedding in a polyester resin and microtomed $\left.{ }^{82}\right]$. The second case shows a study of the distribution of poly(acrylic acid), PAA in laminates of poly(vinyl alcohol), PVOH and nanofibrillated cellulose, NFC [ $\left.{ }^{83}\right]$. The crosslinking agent PAA was shown to migrate out of the PVOH into the nanocellulose layer leading to a PAA-depleted region near the NFC layer, which will result in a weak interphase in the composite. 
Figure 12. Top. Multibeam 2D imaging at the IRENI infrared imaging beamline, SRC. (a) Schematic of the beamline, (b) $128 \times 128$ pixel FPA image with 12 overlapping beams illuminating an area of $50 \times 50 \mu \mathrm{m}^{2}$. Scalebar $=40 \mu \mathrm{m}$, (c) visible light of the 12 projected beams from a screen placed at position marked in the beampath. Scalebar $=15 \mathrm{~mm}$. (d) Long-exposure photograph of the combination of the beams to a single overlapped beam by mirrors $M 3$ and $M 4$. Scalebar $=20 \mathrm{~cm}$. Reprinted by permission from Macmillan Publishers Ltd: Nature Methods, M.J. Nasse, et al., Nature Methods, 8, 413-418 (2011), (C)2011. Bottom left. Imaging of a multilayer polymer film. (a) Visible and (b-e) chemical images of the layered polymer sample. The layers are colour coded red, purple, blue and green for the mounting polyester resin, CTFE, polyester and polyethylene, respectively to correspond to the spectra on the right of the images ( $f$ ); boundaries between layers are denoted with dashed black lines and labeled in the visible image at far left. Chemical images are generated from raw data, based on the peak positions highlighted in the stack of spectra over the integration ranges (b) 1053-1090 $\mathrm{cm}^{-1}$ (polyester mounting resin), (c) 1180-1221 cm (CTFE), (d) 2829-2866 cm-1 (PE) and (e) 1335-1352 cm-1 (polyester). Reproduced from M. Unger, et. al, Appl Phys A (2013) 111:135-145 with permission of Springer, (C) Springer-Verlag Berlin Heidelberg 2012. Bottom right. Images of a PVOH:NFC laminate containing PAA (in an 8:1 PVOH:PAA ratio) showing the different chemical compositions in the layers. (a) Visible image and IR images of (b) NFC (COC band at $1163 \mathrm{~cm}^{-1}$ ), (c) PAA (C=O band at $1715 \mathrm{~cm}^{-1}$ ), (d) PVOH ( $\mathrm{CH}$ band at $\left.1715 \mathrm{~cm}^{-1}\right)$, (f) intensity profiles of these bands along the lines marked in the images for samples with different PVOH:PAA ratios. Reprinted from C. Clemons, et al. Polymer 54 (2013) 2058-2061 C2013 with permission from Elsevier.

The SRC synchrotron facility has since been decommissioned, but the IRENI endstation is being moved and coupled anew to the CAMD synchrotron in Louisiana, and will be available again in the future.

One important additional technique arising from this higher-speed imaging development is $3 D$ FTIR tomography $\left.{ }^{84}\right]$. This provides spectrally rich, label-free, nondestructive visualizations of distinctive chemical compositions throughout intact samples. The technique has combined Fourier Transform Infrared (FTIR) spectroscopy with computed tomography (CT) to create a non-destructive 3D imaging technique that provides molecular-level chemical information of unprecedented detail on specimens with no need to stain or alter the specimen. It greatly enhances the capabilities of both FTIR spectroscopy and tomography by creating a "full color" spectro-micro-tomography where colors are assigned by specific chemical identifications or spectral changes. Importantly, each voxel contains a complete spectrum, a wealth of information for advanced spectral segregation techniques such as clustering, neural networks, and principle components analysis. Co-located chemical fingerprints and morphology in 3D reveal internal composition and structure, Figure 13a.

Figure 13. (a) (Left) Perspective view of a tomographic reconstruction of a Zinnia elegans tracheary element inside a polyimide microloop holder. The red color shows the reconstruction of the integrated intensity from the $2357-2376 \mathrm{~cm}^{-1}$ spectral region, where only the sharp edges of the microloop are visualized. (Middle Left) The blue-green colors show the reconstruction of the $3317-3340 \mathrm{~cm}^{-1} \mathrm{spectral}$ region associated with $\mathrm{OH}$ stretching, highlighting the parallel secondary cell wall thickenings. (Lower Left) Reconstructions of this sample were performed at all 816 individual wavelengths measured. The intensity of one voxel in the stem of the polymer microloop were reassembled to extract the spectrum of this one $1.1 \times 1.1 \times 1.1 \mathrm{~mm}^{3}$ voxel showing a polyimide spectrum with quantitative absorbance matching the volume of the voxel. (Right) Absorption spectra and reconstructed images of a volcanic glass sample are shown. The red-brown (blue-green) colors in the reconstructions show the $1790 \mathrm{~cm}^{-1}$ (3494 $\mathrm{cm}^{-1}$ ) absorption regions, that are superimposed. The blue-green is dominated by water 
absorptions (due to the combined $\mathrm{H}_{2} \mathrm{O}$ and $\mathrm{OH}$ stretching vibrations), whereas the red-brown regions are predominately the edge of the strong silicate glass absorption. The white space between has low absorbance at both spectral regions. The lowermost stripe has water and glass superimposed, the middle stripe shows more water around the glass, and the topmost stripe shows that the water-rich region is mostly parallel and beside the strongest glass region. Scale bars are $20 \mu \mathrm{m}$. Reproduced partly from M.C. Martin, et al., Nature Methods, 10, 861-864 (2013) CNature Publishing Group. (b) Overlayed image reconstruction of PHB (purple) and PLA (black) blends. (i) front view (scale bar $=20 \mu \mathrm{m}$ ), (ii) rotated at an angle of $60^{\circ}$ around the vertical axis. (iii) vertical cut through the sample at the centre, (iv) Full reconstruction image of PHB overlaid with the PLA image cut vertically at the center. Adapted and reproduced with permission from M. Unger et al., Vibr. Spectrosc., 75, 169-172 (2014), C2014 Elsevier.

3D FTIR tomographic imaging was also used to study polymer blends, such as a phaseseparated poly(3-hydroxybutyrate)(PHB)/poly(L-lactic acid) (PLA) (50:50 wt\%) polymer blend film $\left[{ }^{85}\right]$, Figure $13 \mathrm{~b}$. Contrary to conventional $2 \mathrm{D}$ imaging techniques, this analytical approach offers the possibility to combine spectral and spatial information by measuring several hundred thousand spectra of laterally resolved sample positions during $360^{\circ}$ rotation of the sample in a relatively short time thereby providing "chemical images" of the three-dimensional blend morphology. The PHB image (purple (high) to white (low) color scale), and the PLA image (black (high) to white (low) color scale) are shown overlapping in several 3D views in the Figure $13 b$.

Another interesting and rapidly developing 2D imaging approach employs the attenuated total reflectance (ATR) technique. In ATR-FTIR spectroscopy the incident IR beam is totally reflected at the interface between a high refractive index prism and the lower index sample, generating an evanescent wave that propagates a very short distance into the sample (typically between $0.2-5 \mu \mathrm{m}$ ), to a penetration depth, $d_{p}$ defined as the distance required for the electric field amplitude to fall to $\mathrm{e}^{-1}$ of its value at the interface between the crystal and the sample, a parameter that varies with the refractive index difference between the ATR crystal $\left(n_{1}\right)$ and the sample $\left(n_{2}\right)$, the angle of incidence of the radiation $(\theta)$, and the wavelength incident radiation corresponding to the band of interest $(\lambda)$ as expressed in Eq.2.

$$
d_{p}=\frac{\lambda}{2 \pi \sqrt{\left(n_{1}^{2} \sin ^{2} \theta-n_{2}^{2}\right)}}
$$

Polymer scientists are well acquainted with ATR-FTIR spectroscopy, as it is probably the most utilised IR technique used due to its enormous sampling versatility. However, this does not come without a price. The requirement for intimate contact between the sample and the ATR crystal in order to obtain a valid spectrum, the low penetration depth (meaning low path length in the sample, low volume and hence low IR absorption), and the variation of $d_{p}$ as a function of the wavelength generate difficulties for many samples, particularly samples with rough or uneven surfaces, or multicomponent systems such as composites or blends, where refractive index differences between components can lead to erroneous data interpretation. Further, applying pressure in order to obtain intimate contact between the ATR crystal and the material under study often distorts the shape and morphology of the sample and can produce mechanically induced structural changes. Notwithstanding, when the type of sample under study can be adequately adapted to these limitations, it becomes a powerful method, and several authors have developed imaging ATR approaches by coupling to FPA detectors. Figure 14a shows a typical scheme for this type of measurement using a macro-ATR approach and a 
conventional globar source for the study of drug release from polymer matrices $\left[{ }^{86}\right]$ and an example of the release of the analgesic Ibroprufen ${ }^{\mathrm{TM}}$ dispersed in a poly(ethylene glycol), PEG matrix as a function of time exposed to water $\left[{ }^{87}\right]$, Figure $14 \mathrm{~b}$, where it was shown that during the dissolution process, the molecularly dispersed drug firstly accumulates in the outer layer of the formulation, where dimers were observed as a prelude to the formation of crystallites, providing important insight to the drug release mechanism. As well as drug release $\left[{ }^{88}\right]$, this technique is having an important impact in other dynamic studies in polymeric materials such as sorption and diffusion processes or hydrolytic degradation $\left[{ }^{89}\right]$, for example.

Figure 14. (a) Schematic representation of macro-ATR imaging method for drug dissolution studies and (b) Macro-ATR FTIR images (size $3.8 \times 5.3 \mathrm{~mm}^{2}$ ) from a PEG/ibuprofen formulation showing the distribution of PEG (top row) and ibuprofen (bottom row) as a function of time during contact with water. (c) Representative IR spectra in the $\mathrm{C}=\mathrm{O}$ stretching region of ibuprofen showing the spectral differenced between molecularly dispersed and crystalline drug. Reproduced with permission from S. G. Kazarian, et al., Macromolecules, 2003, 37, 9866-9872, (American Chemical Society.

A particularly interesting aspect of ATR imaging is the significant improvement in spatial resolution that can be obtained, which is due to the high refractive index of the ATR internal reflection element that provides an image magnification factor, which is of the order of $4 x$ in the case of a Ge (index of refraction $=4$ ) ATR crystal $\left[{ }^{90}\right]$. Thus, the spatial contrast in the resulting IR image will depend on the coupling optics between the surface of the ATR crystal and the FPA, where high spatial contrast images are obtained at the expense of the size of the field of view $\left[{ }^{91}\right]$. Examples of micro-ATR imaging in polymeric materials include the study of polymer/carbon fibre interfaces and the effect of a compatibilising agent in immiscible polymer blends $\left[{ }^{86}\right]$. In practical terms, with a synchrotron source sub-micron imaging becomes available when considering spectroscopic features in the high wavenumber region $(\mathrm{C}-\mathrm{H}, \mathrm{O}-\mathrm{H}$, $\mathrm{N}-\mathrm{H}$ stretching region) of the samples under study. Many IR synchrotron beamlines now offer or are developing ATR imaging approaches, which may be of considerable interest in polymer research, particularly for following dynamic processes. In this respect, of particular interest is the coupling of ATR imaging to chip-based microfluidic devices $\left[{ }^{92}\right]$ paving the way to further developments for the study of controlled chemical processes in nanovolumes $\left[{ }^{93}\right]$, or the adsorption of polymer molecules to surfaces under flow fields $\left[{ }^{94}\right]$.

The development of microfluidic devices for IR microspectroscopy has essentially been driven by the biological sciences with the challenge to obtain FT-IR data from aqueous solutions or living biological cells, the major difficulties being due to the competition between the water film thickness and the materials under study. In this respect, ATR-FT-IR imaging is a promising approach that allows cells to be maintained in a thicker layer of water $\left[{ }^{95}\right]$, but is limited to measuring only materials that are in close contact with the ATR crystal due to the small penetration depth of the evanescent wave. Many synchrotron facilities now incorporate microfluidics options (see Table 1), and there is a growing expertise in lithographic and templating methods using IR transparent materials. There are numerous studies employing synchrotron IR microspectroscopy and imaging particularly for long-term live-cell studies including bacterial activity, cell growth and proliferation and cell - drug interactions $\left[{ }^{96}\right]$, but to date no imaging synchrotron IR microfluidics studies on polymeric systems have been reported. However, this is an area with enormous future possibilities, particularly for the study of diverse phenomena such as flow-induced orientation and crystallization in dilute solutions, 
polymerisation reactions and kinetics or microparticle synthesis and assembly [ $\left.{ }^{97}\right]$. Indeed, very recently confocal Raman spectroscopy has been applied to on-line monitoring of solution and miniemulsion polymerisation $\left[{ }^{98}\right]$, and $x$-ray microspectroscopy (NEXAFS) and SIRMS imaging have been combined in the study of catalyis of organic reactions in microflow reactors $\left[{ }^{99}\right]$.

Continued development of pixelated detectors optimized for the high-brightness of synchrotron sources and further improvements in beamline sources should yield ever more capable wide-field rapid imaging facilities and instrumentation. The coupling of this enormous capability to controlled micro and nanoscale sampling enviroments, such as chip-based minireactors or microfluidics devices, will provide a plethora of opportunities for real-time studies in polymeric materials, from polymer synthetic chemistry, to interactions at the polymerbiomaterial interface, or transformation processes such as self-assembly, crystallization, conformational rearrangements or degradation induced by external factors (heat, light, shear, etc.).

\section{Beyond the diffraction limit}

Science on the nanoscale is characterized by new physical and chemical properties of matter that emerge at dimensions intermediate between those of individual atoms or molecules and the homogeneous bulk. Phase-separated polymers and copolymers, nanostructured materials, micelle formation, lipid bilayer membranes, supramolecular assemblies, protein and DNA folding are all examples of intra- or intermolecular self-assembly associated with an increase in the internal degree of organization of the system. The chemical specificity of infrared vibrational spectroscopy has early on generated the desire for simultaneous spatial resolution to determine the microscopic composition of a sample. But nanometer resolutions are required to probe many systems of high interest and application. Optical near-field techniques are thus needed to reach beyond the far-field diffraction limit.

Near-field IR nanospectroscopy was pioneered by combining scattering-scanning near-field optical microscopy (s-SNOM) with tuneable IR laser sources in an infrared spectrometer $\left[{ }^{100}\right]$, can achieve spatial resolution of the order of $20 \mathrm{~nm}\left[{ }^{101}\right]$ and has since developed into a powerful and commercially available technique. Synchrotron Infrared Nano-Spectroscopy (SINS) is the combination of s-SNOM with infrared synchrotron radiation, and enables broadband molecular and phonon vibrational spectroscopic imaging with rapid spectral acquisition over the full mid-infrared $\left(500-5000 \mathrm{~cm}^{-1}\right)$ region with nanoscale spatial resolution [102]. Indeed, as with all tip based methods, the spatial resolution is no longer wavelengthdependent, but is instead only a function of how sharp the AFM tip is. This highly powerful combination provides access to a qualitatively new form of nano-chemometric analysis with the investigation of nanoscale, mesoscale, and surface phenomena that were previously impossible to study with IR techniques. Naturally, because of the use of an AFM tip the SINS technique is more surface sensitive and samples must be sufficiently flat as for most scanning probe techniques. The SINS arrangement at Beamline 5.4 at the ALS at Lawrence Berkeley National Laboratory is shown in Figure 15a, and its performance on semiconductor, biomineral, protein nanostructures, and more have demonstrated vibrational chemical imaging with sub-zeptomole sensitivity. The peptoid nanosheet imaged by SINS in Figure 15b is $<8 \mathrm{~nm}$ thick at some points, showing that it possible to acquire spectroscopic images of these ultra-thin nanosheets for the first time. 
Figure 15. Synchrotron infrared nanospectroscopy (SINS) (a) Experimental setup for SINS at beamline 5.4 of the ALS, and (b) SINS image of a peptoid nanosheet. (c) SINS and IR broadband imaging of PMMA microcrater on Au recorded at LNLS, Brasil. $a=$ AFM topography and $b=$ IR broadband image of $a$ micrometer size crater-like structure formed by PMMA spin-coated on the Au substrate (scale bars represent $500 \mathrm{~nm}$ ). The central rounded feature (lower topography and higher optical signal) is the exposed Au substrate. $c=$ topography and SINS intensity profiles along the dashed line in $a$ and $b$ showing the relation of the IR broadbandsignal with the thickness of the PMMA film, $d=$ SINS recorded from PMMA (at $\diamond$ mark) normalized by a reference spectrum taken on Au (at $\star$ mark), e = macroscopic far-field ATR-FTIR absorbance of the same sample. Reproduced with permission from $B$. Pollard, et al., Nano Lett. 2016, 16, 55-61, (c) American Chemical Society. (d) Sample scans of a quasilamellar poly(styrene)/poly(methyl methacrylate) block copolymer (PS-b-PMMA). a = AFM tapping mode, $b=$ AFM height, $c=$ near-field phase (fs-SNOM) on-resonance, and $b=$ off-resonance with the carbonyl mode of MMA. Scale bars $200 \mathrm{~nm}$. Reproduced with permission from B. Pollard, et al., Nature Comm. 2014, 5, 3587, (C) Macmillan Publishers Ltd.

The IR nanospectroscopy technique is available to users or is being introduced at several synchrotron facilities, where beamlines already have (or are developing) AFM/s-SNOM or photothermal-induced resonance (PTIR) methods $\left[{ }^{103}\right]$, the latter now implemented at Diamond Light Source, UK $\left[{ }^{104}\right]$. Although SINS or PTIR measurements on polymers have, to date, been limited to optimising the technique, an example from PMMA is given in Figure 15c $\left[{ }^{105}\right]$, successful measurements of nanoscale morphology associated with intermolecular interactions in polymer blends via s-SNOM using infrared quantum cascade laser (QCL) sources $\left[{ }^{106}\right]$, Figure $15 d$, demonstrate the tremendous potential of the synchrotron-based methods, which will rapidly advance in the forthcoming years.

An exciting aspect of the SINS technique pointed out earlier is that the spatial resolution is only dependent on the AFM tip, meaning that it will continue to work even outside the mid-IR spectral range, and indeed it has been recently shown to work in the far-IR using a Si:B detector at the ALS. THz scanning near-field imaging to using coherent synchrotron radiation (CSR) $\left[{ }^{107}\right]$ was pioneered at BESSY-II synchrotron $\left[{ }^{108}\right]$ with apertureless probes, demostrating spatial resolutions better than $\lambda / 40$ at $2 \mathrm{~cm}^{-1}$. Several applications of synchrotron near-field $\mathrm{THz}$ microspectroscopy have been described in biological systems and semiconductor materials $\left[{ }^{108}\right]$ and minerals $\left[{ }^{109}\right]$, but to date no work has been done on polymeric materials. Of course $\mathrm{THz}$ time domain spectroscopy (TDS) is an interesting technique to obtain information on the dielectric properties (refractive index, absorption, dielectric functions) of polymers $\left[{ }^{110}, 111\right]$ and as a tool to measure the glass transition temperature [112]. The spatial and intensity advantage using a synchrotron near-field approach is clearly a powerful future approach, especially in complex multiphase or hierarchical composite systems.

\section{Special environments. High pressure.}

The small size of the synchrotron beam is also a fundamental advantage for the study of very small particles, and this has been clearly demonstrated in other areas such as astrophysics or cultural heritage materials where often the amount of material available is inevitably very small. However, another advantage of the small synchrotron footprint is in extreme environments, such as high pressure. In IR microspectroscopy, several types of pressure cells 
are available, the most effective being diamond anvil cells that are highly transparent in the IR, can be conveniently coupled to commercial cryostats and temperature control, and can reach extremely high pressures. Clearly the pressure range required defines the maximum size of the anvil, and the higher the pressure, the smaller the available aperture to illuminate the sample, hence the need for a synchrotron source. IR spectra have recently been recorded in the study of hydrogen using SIRMS at pressures up to $300 \mathrm{GPa}\left[{ }^{113}\right]$, recorded through $6 \times 6 \mu \mathrm{m}^{2}$ apertures in a specially designed diamond anvil with only a $10 \mu \mathrm{m}$ diameter clear window. It is well accepted that new and powerful chemistry is available under extreme pressure environments $\left[{ }^{114}\right]$, as the transformation of coal into diamond at the extreme conditions found within the earth attest. There is extensive literature available on the study of the effect of pressure on polymers at relatively low pressure, more relevant to polymer processing, $<1 \mathrm{GPa}$, but there are only few spectroscopic studies available on polymers under high pressure conditions, where phenomena such as new phase transitions, amorphitization of semicrystalline polymers, shock compression or pressure-induced crosslinking have been studied [ $\left.{ }^{115}\right]$. Recently, Cavallo et al. has undertaken a series of preliminary SIRMS measurements from different crystalline polymorphs of isotactic polypropylene at hydrostatic pressures between $0-20 \mathrm{GPa}$, and has observed interesting behaviour from the regularity bands that appear to suggest differential pressure effects on the long and short helical sequence distributions as a function of the initial crystalline structure $\left[{ }^{116}\right]$. As an example Figure 16a shows the effect of high pressure on the IR spectra of a film of monoclinic ( $\alpha$-form) iPP, recorded through a $15 \times 15 \mu \mathrm{m}^{2}$ aperture. All of the vibrational bands are seen to inhomogeneously broaden, shift to higher frequency, and decrease in intensity as the pressure is increased up to $11 \mathrm{GPa}$ where amorphitization sets in. The subtle changes in the regularity bands indicated variations in the distribution of long and short helical sequences, and whilst no evidence for conformational changes were found, complementary synchrotron $\mathrm{x}$-ray microdiffraction measurements will soon be undertaken to obtain a more complete picture of nature of these variations and will be reported in due course.

In contrast to the case for polymers, high-pressure studies of small organic molecules abound, as these can undergo a wide range of conformational changes, phase transitions and bonding regimes. Many examples of high pressure polymerization have been reported $\left[{ }^{117}\right]$, and there is recent interest in this area for both the formation of traditional polymers using alternative methods $\left.{ }^{118}\right]$ or for the formation of novel composite materials with tailored properties: An example of this is a unique organic/inorganic conducting nanocomposite fabricated by polymerizing acetylene at high pressure in a microporous $\mathrm{SiO}_{2}$ zeolite framework $\left.{ }^{119}\right]$. The nanocomposite, denominated PASIL, comprised of conjugated chains of polyacetylene, PA embedded in the silicalite, was formed by simply applying a pressure of approximately $4 \mathrm{GPa}$ and was completely recoverable at ambient pressure. Spectroscopic information from both SIRMS and Raman microspectroscopy, combined with x-ray diffraction measurements provided important information on confinement effects on the polymerization of acetylene, showing a prevalence for highly branched short chains, see Figure $16 \mathrm{~b}$.

Figure 16. High pressure studies using SIRMS. (a) SIRMS spectra of iPP recorded through $15 \times 15 \mu \mathrm{m}^{2}$ apertures in a DAC. Data courtesy of D. Cavallo, University of Genova, Italy (see Ref. 116); (b) IR absorption spectra of bulk (lower) and nanoconfined (upper) polyacetylene in PASIL in C-H stretching mode region, at $2.7 \mathrm{Gpa}$. Conjugated chains are characterised by the $\mathrm{C}\left(\mathrm{sp}^{2}\right)-\mathrm{H}$ peaks (green), whereas branching and chain terminations by the $\mathrm{C}\left(\mathrm{sp}^{3}\right)-\mathrm{H}$ peaks (blue). Absorbances of the two spectra were normalized to the same integrated intensity of combination bands of residual acetylene, (c) 3D model of 
PASIL composite. Reproduced with permission from D. Scelta, et al., Chem. Mater. 2014, 26, 2249-2255, (C) American Chemical Society.

Thus, there is a fundamental opportunity to apply SIRMS to the study of many relevant polymeric materials at high pressure, since diamond anvil cells are becoming standard sampling tools at several synchrotron beamlines (see Table 1) to achieve pressures of $20 \mathrm{GPa}$ or higher. With a bright and tightly focused synchrotron beam both single point spectra and mapping now become available, and spatially resolved measurements on heterogeneous or isotropic materials can be undertaken.

\section{Conclusions}

The world has a growing number of synchrotron infrared beamlines and their use has also rapidly grown across a wide number of scientific applications. Whilst this vision of the possibilities of SIRMS is far from comprehensive, we hope it has highlighted several of the exciting recent developments in the field and the opportunities available for the application of these sources to a wide variety of polymer science. In the near future we anticipate several advances, which will further increase the uses and capabilities of synchrotron-based infrared spectroscopies and microscopies. To find out more about how synchrotron infrared techniques may play a role in your research, we encourage you to contact one of the many friendly infrared beamline scientists at a synchrotron light source near you.

\section{Acknowledgements}

The authors would like to thank Paul Dumas and Mark Tobin for the data provided for Figure 4, and Dario Cavallo for the data in Figure 16a, obtained at the SMIS beamline at SOLEIL Synchrotron, and some of the data in Figure 8 was obtained at the IRIS beamline at the Helmholtz-Zentrum Berlin BESSY-II synchrotron, both through Projects funded by the European Community's Seventh Framework Programme (FP7/2007-2013) under grant agreement n. ${ }^{\circ}$ 226716. MCM acknowledges that the Advanced Light Source is supported by the Director, Office of Science, Office of Basic Energy Sciences, of the U.S. Department of Energy under Contract No. DE-AC02-05CH11231. Finally, we acknowledge colleagues from various synchrotron facilities for providing information that allowed us to construct Table 1 . The synchrotron infrared community is very open and collaborative, and it is a pleasure to collaborate with so many outstanding colleagues. 


\section{References}

${ }^{1}$ a) E.R. Blout, G.R. Bird Infrared Microspectroscopy. J. Opt. Soc. Am. 40, 304-313 (1950); b)

E.R. Blout, G.R. Bird Infrared Microspectroscopy II. J. Opt. Soc. Am. 41, 547-551 (1951)

${ }^{2}$ a) R.G. Messerschmidt, M.A. Harthcock (Eds.), Infrared Microscopy, Theory and Applications, Practical Spectroscopy Series, vol. 6, Marcel Dekker, New York, 1988; b) H. J. Humecki, Practical Guide to Infrared Microspectroscopy, CRC Press, 1995; c) J. L. Koenig, Microspectroscopic Imaging of Polymers, American Chemical Society, 1988, ISBN: 084123493; d) R. Bhargava, S.Q. Wang, J.L. Koenig, FTIR Microspectroscopy of Polymeric Systems, Adv Polym Sci (2003) 163: 137-191

${ }^{3}$ a) J. A. Reffner, P. A. Martoglio, G. P. Williams. Fourier transform infrared microscopical analysis with synchrotron radiation: The microscope optics and system performance (invited). Rev. Sci. Instrum. 66, 1298 (1995); b) G.L. Carr, J.A. Reffner, G.P. Williams, Performance of an infrared microspectrometer at the NSLS. Rev. Sci. Instrum. 66, 1490 (1995)

${ }^{4}$ G.L. Carr, P. Dumas, C.J. Hirschmugl, G.P. Williams. Infrared synchrotron radiation programs at the National Synchrotron Light Source. II Nuovo Cimento 20, 375 (1998)

${ }^{5}$ K. Araki, N. Yagi, Y. Ikemoto, H. Yagi, C.-J. Choong, H. Hayakawa, G.Beck, H. Sumi, H. Fujimura, T. Moriwaki, Y. Nagai, Y. Goto, H. Mochizuki, Synchrotron FTIR micro-spectroscopy for structural analysis of Lewy bodies in the brain of Parkinson's disease patients, Scientific Reports 5, 17625 (2015)

${ }^{6}$ P. Dumas, N. Jamin, J. L. Teillaud, L. M. Miller and B. Beccard, Imaging capabilities of synchrotron infrared microspectroscopy, Faraday Discuss. , 126, 289-302 (2004)

${ }^{7}$ M. C. Martin, N. M. Tsvetkova, J. H. Crowe and W. R. McKinney, Negligible Sample Heating from Synchrotron Infrared Beam, Appl. Spectrosc., 55, 111 (2001)

8 P. Dumas, G.L. Carr, G.P. Williams, Enhancing the lateral resolution in infrared microspectrometry by using synchrotron radiation: applications and perspectives. Analusis, Eur. J. Anal. Chem. 28, 68 (2000)

${ }^{9}$ G.L. Carr, Resolution limits for infrared microspectroscopy explored with synchrotron radiation. Rev Sci Instrum 72 (3), 1613-1619 (2001)

${ }^{10}$ a) P. Lasch, D. Naumann, Spatial resolution in infrared microspectroscopic imaging of tissues. Biochimica et Biophysica Acta 1758, 814-829 (2006) ; b) E. Levenson, P. Lerch, M. C. Martin, Spatial resolution limits for synchrotron-based spectromicroscopy in the mid- and nearinfrared, J. Synchrotron Rad. 15, 323-328 (2008); c) H.A. Bechtel, M.C. Martin, T. E. May, and P. Lerch, Improved spatial resolution for reflection mode infrared microscopy, Rev. Sci. Instrum. 80, 126106 (2009)

${ }^{11}$ K. Katayama, S. Tanase, M. Ohta, K. Tanaka, T. Konakazawa, N. Ishihara, Y. Ikemoto, T. Nishioka. Analysis of composition distribution in high impact polypropylene particles using synchrotron infrared microspectroscopy imaging. Bunsei Kagaku, 59, 531 (2010)

${ }^{12} \mathrm{~K}$. Katayama, S. Tanase, N. Ishihara. Considerations on detailed analysis and particle growth in high impact polypropylene particles, J. Appl. Polym. Sci. 122, 632-638 (2011)

${ }^{13}$ J.A. Debling, W. Harmon Ray, Morphological development of impact polypropylene produced in gas phase with a TiCl4/MgCl2 catalyst J. Appl. Polym. Sci., 81, 3085 (2001)

${ }^{14}$ D. Muscat, R. Adhikari, M. J. Tobin, S. McKnight, L. Wakeling, B. Adhikari, Effect of spatial distribution of wax and PEG-isocyanate on themorphology and hydrophobicity of starch films, Carbohydrate Polymers 111, 333- 347 (2014)

${ }^{15}$ D. Muscat, M. J. Tobin, Q. Guo, B. Adhikari, Understanding the distribution of natural wax in starch-wax films using synchrotron-based FTIR (S-FTIR) Carbohydrate Polymers 102, 125- 135 (2014)

${ }^{16}$ R.J. Schexnaydre, B.S. Mitchell, Synchrotron infrared microspectroscopy characterization of heterogeneities in solid-state blended polymers, Materials Letters, 61, 2151-2155 (2007)

${ }^{17}$ G. Ellis , C. Marco , M. A. Gómez , E.P. Collar, J.M. García-Martínez, The Study of Heterogeneous Polymer Systems by Synchrotron Infrared Microscopy, Journal of Macromolecular Science, Part B, 43, 253-266 (2004) 
${ }^{18}$ R. A. Russell, T. A. Darwish, L. Puskar, D. E. Martin, P. J. Holden, L.J.R. Foster. Deuterated Polymers for Probing Phase Separation Using Infrared Microspectroscopy. Biomacromol., 15, 644-649 (2014)

${ }^{19}$ G. Ellis, P. Cano, M. Jadraque, M. Martín, L. López, T. Núñez, E. de la Peña, C. Marco, L. Garrido, Laser microperforated biodegradable microbial polyhydroxyalkanoate substrates for tissue repair strategies: an infrared microspectroscopy study, Anal Bioanal Chem. 399, 23792388 (2011)

${ }^{20}$ D. Saviello, E. Pouyet, L. Toniolo, M. Cotte, A. Nevin. Synchrotron-based FTIR microspectroscopy for the mapping of photo-oxidation and additives in acrylonitrilebutadiene-styrene model samples and historical objects. Anal. Chim. Acta 843, 59-72 (2014)

${ }^{21}$ M. Maric, W. van Bronswijk, S.W. Lewis, Kari Pitts, D. E. Martin, Characterisation of chemical component migration in automotive paint by synchrotron infrared imaging, Forensic Sci.Int. 228, 165-169 (2013)

${ }^{22}$ M. Maric, W. van Bronswijk, S.W. Lewis, Kari Pitts, Synchrotron FTIR characterisation of automotive primer surfacer paint coatings for forensic purposes, Talanta, 118, 156-161 (2014)

${ }^{23}$ K.Aardahl, S.Kirkowski, R.D.Blackledge, Science and Justice, 45, 7-12 (2005)

${ }^{24}$ L.Vernoud, H.A. Bechtel, M.C. Martin, J.A.Reffner, R.D.Blackledge, Forensic Sci. Int., 210, 4751 (2011)

25 J-P. Veder, K. Patel, G. Clarke, E. Grygolowicz-Pawlak, D.S. Silvester, R. De Marco, E. Pretsch, E. Bakker. Synchrotron Radiation/Fourier Transform-Infrared Microspectroscopy Study of Undesirable Water Inclusions in Solid-Contact Polymeric Ion-Selective Electrodes. Anal. Chem., $82,6203-6207$ (2010)

${ }^{26}$ D.A. Beattie, A. Beaussart, A. Mierczynska-Vasilev, S.L. Harmer, B. Thierry, L. Puskar, M. Tobin. Synchrotron FTIR Microscopy of Langmuir-Blodgett Monolayers and Polyelectrolyte Multilayers at the Solid-Solid Interface. Langmuir, 28, 1683-1688 (2012)

${ }^{27}$ L. F.Dumée, F. M. Allioux, R.Reis, M. Duke, S. Gray, M. Tobin, L. Puskar, L. He, P. Hodgson, L. $X$. Kong. Qualitative spectroscopic characterization of the matrix-silane coupling agent interface across metal fibre reinforced ion exchange resin composite membranes. Vibrat. Spectrosc., 75, 203-212 (2014)

${ }^{28}$ T. Endale, E. Sovernigo, A. Radivo, S. Dal Zilio, A. Pozzato, T. Yohannes, L. Vaccari, M. Tormen. Investigation of photodegradation in polymer solar cells blended with different fullerenes derivatives. Solar Energy Mater. \& Solar Cells, 123, 150-158 (2014)

${ }^{29}$ V. Barna, E.S. Barna, Synchrotron infrared microspectroscopy of nematic liquid crystals in polymeric micro cavities. Optoelectr. Adv. Mater. - Rapid Comm., 5, 1046-1049 (2011)

${ }^{30}$ P. Dumas, L. M. Miller, and M. J. Tobin, Challenges in biology and medicine with synchrotron infrared light, Acta Phys. Pol. A 115, 446 (2009)

${ }^{31}$ G. Santoro, I. Yousef, F. Jamme, P. Dumas, G. Ellis, Infrared synchrotron radiation from bending magnet and edge radiation sources for the study of orientation and conformation in anisotropic materials, Rev. Sci. Instrum. 82, 033710 (2011)

${ }^{32}$ G. Ellis, C. Marco, M.A. Gómez, Synchrotron Infrared Microscopy Study of the Crystalline Morphology of the Interphase in Polypropylene/LCP-Fiber Model Composites J. Macromol. Sci. Phys. B43, 191-206 (2004)

33 J. Torre, M. Cortazar, M.A. Gomez, C. Marco, G. Ellis, P. Dumas, C. Riekel, Macromolecules 39, 5564-5568 (2006)

${ }^{34}$ a) J. Varga, J. Karger-Kocsis, Interfacial morphologies in carbon fibre-reinforced polypropylene microcomposites, Polymer, 36, 4877 (1995); b) J. Varga, J.Karger-Kocsis, Rules of supermolecular structure formation in sheared isotactic polypropylene melts, J. Polym. Sci., Part B: Polym. Phys., 34, 657 (1996)

${ }^{35}$ G. Santoro, I. M. Ochando, G. Ellis, Advanced Vibrational Microspectroscopic Study of Conformational Changes within a Craze in Poly(ethylene terephthalate), Macromolecules, 48, 1162-1168 (2015)

${ }^{36} \mathrm{C}$. Marcott, Linear dichroism of polymer films using a polarization modulation Fourier transform infrared technique, Appl. Spectrosc. 38, 442-443 (1984) 
${ }^{37}$ Y. Shigematsu, A. Takada, N. Nemoto, K.H. Nitta, An instrument for simultaneous kinetic measurements of microscopic infrared dichroism and stress of inhomogeneous polymer thin films at constant elongation rate, Rev. Sci. Instrum. 72, 3927-3932 (2001)

${ }^{38}$ M. Schmidt, U. Schade, M. Grunze, Microspectroscopic observation of vibrational linear dichroism using polarization-modulated infrared synchrotron radiation, Infrared Phys.

Technol., 49, 69-73 (2006)

${ }^{39}$ T. Buffeteau, B. Desbat, M. Pézolet, J.M. Turlet, Mesure de l'orientation des polymères en dichroïsme linéaire infrarouge par modulation de polarisation: procédure expérimentale et analyse quantitative, J. Chim. Phys. 90, 1467-1489 (2006)

${ }^{40}$ M. Pézolet, C. Pellerin, R. E. Prud'homme, T. Buffeteau, Vibrational Spectroscopy, 18, 103110 (1998)

${ }^{41}$ T. Buffeteau, M. Pézolet, Linear Dichroism in Infrared Spectroscopy, in J.M. Chalmers and

P.R. Griffiths (Eds.) Handbook of Vibrational Spectroscopy, Vol. 1, pp.693-710 (2002) John

Wiley \& Sons Ltd, Chichester UK. ISBN: 0-471-98847-2

${ }^{42}$ M. Schmidt, N. Gierlinger, U. Schade, T. Rogge, M. Grunze Polarized Infrared

Microspectroscopy of Single Spruce Fibers: Hydrogen Bonding in Wood Polymers, Biopolymers, Vol. 83, 546-555 (2006)

${ }^{43}$ G. Santoro, M. Schmidt, U. Schade, C. Marco, G. Ellis, Polarization-modulated synchrotron infrared microspectroscopy for the study of crystalline morphology in some semicrystalline polyolefins J. Phys. Conf. Ser., 359, 012005 (2012)

${ }^{44}$ B. Crist, J.M. Schultz, Polymer Spherulites: A Critical Review, Prog. Polym. Sci. (2015)

[accepted manuscript] DOI: 10.1016/j.progpolymsci.2015.11.006

45 a) H.D. Keith Banding in Polymer Spherulites: Two recurring topics, Polymer 42, 9987-9993 (2001); b) E. M. Woo, G. Lugito, Origins of periodic bands in polymer spherulites, European Polymer Journal, 71, 27-60 (2015)

${ }^{46} \mathrm{H}$. D. Keith and F. J. Padden, Jr., Twisting orientation and the role of transient states in polymer crystallization, Polymer, 25, 28-42 (1984)

${ }^{47}$ P.J. Barham, A. Keller, E.L. Otun, P.A. Holmes, Crystallization and morphology of a bacterial thermoplastic: poly-3-hydroxybutyrate), J. Mater. Sci., 19, 2781-2794 (1984)

${ }^{48}$ M. Gazzano, M.L. Focarete, C. Reikel, M. Scandola, Biomacromol., 1, 604 (2000)

${ }^{49}$ M. Gazzano, M.L. Focarete, C. Reikel, A. Ripamonti, M. Scandola, Macromol. Chem. Phys., 202, $1405(2001)$

${ }^{50} \mathrm{C}$. Bungay, T.E. Tiwald, Infrared spectroscopic ellipsometry study of molecular orientation induced anisotropy in polymer substrates, Thin Solid Films 455-456, 272-277 (2004)

${ }^{51}$ K. Hinrichs, M. Gensch, A. Röseler, E. H. Korte, K. Sahre, K.-J. Eichhorn, N. Esser, U. Schade, Fourier Transform Infrared Synchrotron Ellipsometry for Studying the Anisotropy of Small Organic Samples, Appl. Spectrosc. 57, 1250-1253 (2003)

${ }^{52}$ K. Hinrichs, A. Röseler, M. Gensch, E.H. Korte, Structure analysis of organic films by midinfrared ellipsometry, Thin Solid Films 455-456, 266-271 (2004)

${ }^{53}$ K. Hinrichs, D. Aulich, L. Ionov, Norbert Esser, K.-J. Eichhorn, M. Motornov, M. Stamm, S. Minko, Chemical and Structural Changes in a pH-Responsive Mixed Polyelectrolyte Brush Studied by Infrared Ellipsometry, Langmuir, 25, 10987-10991 (2009)

${ }^{54}$ D. Aulich, O. Hoy, I. Luzinov, M. Brücher, R. Hergenröder, E. Bittrich, K.-J. Eichhorn, P. Uhlmann, M. Stamm, N. Esser, K. Hinrichs, In Situ Studies on the Switching Behavior of Ultrathin Poly(acrylic acid) Polyelectrolyte Brushes in Different Aqueous Environments, Langmuir, 26, 12926-12932 (2010)

${ }^{55}$ D. Aulich, O. Hoy, I. Luzinov, K.-J. Eichhorn, M. Stamm, M. Gensch, U. Schade, N. Esser, K. Hinrichs, In-situ IR synchrotron mapping ellipsometry on stimuli-responsive PAA-b-PS/PEG mixed polymer brushes, P hys. Status Solidi C 7, 197-199 (2010)

${ }^{56}$ A. Furchner, E. Bittrich, P. Uhlmann, K.-J. Eichhorn, K. Hinrichs, In-situ characterization of the temperature-sensitive swelling behavior of poly( $\mathrm{N}$-isopropylacrylamide) brushes by infrared and visible ellipsometry, Thin Solid Films, 541, 41 (2013)

${ }^{57}$ A. Kroning, A. Furchner, Dennis Aulich, E. Bittrich, S. Rauch, Petra Uhlmann, K.-J. Eichhorn, M. Seeber, I. Luzinov, S. M. Kilbey II, Bradley S. Lokitz, S. Minko, K. Hinrichs, In Situ Infrared 
Ellipsometry for Protein Adsorption Studies on Ultrathin Smart Polymer Brushes in Aqueous Environment, ACS Appl Mater Interfaces, 7, 12430 (2015)

${ }^{58}$ M. Gensch, E.H. Korte, N. Esser, U. Schade, K. Hinrichs, Microfocus-infrared synchrotron ellipsometer for mapping of ultra thin films, Infrared Phys. Technol. 49, 74-77 (2006)

${ }^{59}$ K. Hinrichs, M. Gensch, N. Esser, U. Schade, J. Rappich, S. Kröning, M. Portwich, R. Volkmer, Analysis of biosensors by chemically specific optical techniques. Chemiluminescence-imaging and infrared spectroscopic mapping ellipsometry, Anal. Bioanal. Chem. 387,1823-1829 (2007)

${ }^{60}$ K. Hinrichs, A. Furchner, Jörg Rappich, T. W. H. Oates, Polarization-Dependent and Ellipsometric Infrared Microscopy for Analysis of Anisotropic Thin Films, J. Phys. Chem. C, 117, 13557-13563 (2013)

${ }^{61}$ a) G. Birarda, G. Grenci, L. Businaro, B. Marmiroli, S. Pacor, F. Piccirilli, L. Vaccari, Infrared microspectroscopy of biochemical response of living cells in microfabricated devices, Vibr. Spectrosc., 53, 6-11 (2010); b) L. Quaroni, T. Zlateva, E. Normand, Detection of Weak Absorption Changes from Molecular Events in Time-Resolved FT-IR Spectromicroscopy Measurements of Single Functional Cells. Anal. Chem., 83, 7371-7380 (2011)

${ }^{62}$ E. Stavitski, M.H.F. Kox, I. Swart, F.M.F. de Groot, B. M. Weckhuysen. In Situ SynchrotronBased IR Microspectroscopy To Study Catalytic Reactions in Zeolite Crystals. Angew. Chem. Int. Ed., 47, $3543-3547$ (2008)

${ }^{63}$ A. Greenaway, B. Gonzalez-Santiago, P.M. Donaldson, M.D. Frogley, G. Cinque, J. Sotelo, S. Moggach, E. Shiko, S. Brandani, R.F. Howe, P.A. Wright. In situ Synchrotron IR Microspectroscopy of $\mathrm{CO}_{2}$ Adsorption on Single Crystals of the Functionalized MOF $\mathrm{Sc}_{2}$ (BDC$\left.\mathrm{NH}_{2}\right)_{3}$. Angew. Chem. Int. Ed., 53, $13483-13487$ (2014)

${ }^{64}$ H.-Y. N. Holman, R. Miles, Z. Hao, E. Wozei, L.M. Anderson, H. Yang, Real-time chemical imaging of bacterial activity in biofilms using open-channel microfluidics and synchrotron FTIR spectromicroscopy Anal. Chem., 81, 8564-8570 (2009)

${ }^{65}$ G. Ellis, G. Santoro, M.A. Gómez, C. Marco, Synchrotron IR microspectroscopy: Opportunities in polymer science, IOP Conf. Series: Materials Science and Engineering, 14, 012019 (2010)

${ }^{66}$ D. Cavallo, L. Zhang, I. Sics, G.C. Alfonso, P. Dumas, C. Marco, G. Ellis. Morphology and polymorphism of self-nucleated trigonal isotactic poly(1-butene) studied by synchrotron IR microspectroscopy, Cryst Eng Comm (2016) [Advance article] DOI: 10.1039/C5CE01727K ${ }^{67}$ F. Danusso, G. Gianotti, Isotactic polybutene-1: Formation and transformation of modification 2. Die Makromol. Chem., 88, 149-158 (1965)

${ }^{68}$ D. Cavallo, G. Alfonso, Concomitant Crystallization and Cross-Nucleation in Polymorphic Polymers. Adv. Polym. Sci. 2015, Springer Berlin Heidelberg, DOI: 10.1007/12_2015_330

${ }^{69}$ D. Cavallo, L. Gardella, G. Portale, A.J. Müller, G.C. Alfonso, Self-nucleation of isotactic poly(1-butene) in the trigonal modification. Polymer, 54, 4637-4644 (2014)

${ }^{70}$ F. Su, X. Li, W. Zhou, S. Zhu, Y. Ji, Z. Wang, Z. Qi, L. Li, Direct Formation of Isotactic Poly(1butene) Form I Crystal from Memorized Ordered Melt. Macromolecules, 46 , 7399-7405 (2013) ${ }^{71}$ a) M.J. Romeo, S. Boydston-White, S.; C. Matthäus, M. Milijovic, B. Bird, T. Chernenko, M. Diem, Vibrational microspectroscopy of cells and tissues, Chapter 6, pp.121-152, in "Biomedical Vibrational Spectroscopy" (Eds. P. Lasch, J. Kniepp), John Wiley \& Sons (2008); b) M. Diem, "Modern Vibrational Spectroscopy and Micro-Spectroscopy: Theory, Instrumentation and Biomedical Applications", John Wiley \& Sons (2015).

${ }^{72}$ E. N. Lewis, P.J. Treado, R. C. Reeder, G. M. Story, A. E. Dowrey, C. Marcott, , I. W. Levin, Fourier Transform Spectroscopic Imaging Using an Infrared Focal-Plane Array Detector, Anal. Chem., 67, 3377-3381 (1995)

${ }^{73} \mathrm{G}$. Steiner, E. Koch, Trends in Fourier transform infrared spectroscopic imaging, Anal. Bioanal. Chem., 394, 671-678 (2009)

${ }^{74}$ H. W. Siesler (Ed.) "Infrared and Raman Spectroscopic Imaging", John Wiley \& Sons (2014) ISBN: 3527678158

${ }^{75}$ R. Bhargava, S.Q. Wang, J.L. Koenig, FTIR Microspectroscopy of Polymeric Systems, Adv. Polym. Sci. 163, 137-191 (2003)

${ }^{76}$ E. Levenson, P. Lerch, M.C. Martin, Infrared imaging: synchrotrons vs. arrays, resolution vs. speed, Infrared Phys. Technol. 49, 45-52 (2006) 
77 L. M. Miller, P. Dumas, Chemical imaging of biological tissue with synchrotron infrared light, Biochim. Biophys. Acta, 1758, 846 (2006)

${ }^{78}$ G.L. Carr, L.M. Miller, P. Dumas in D. Moss D (Ed.) Biomedical Applications of Synchrotron Infrared Microspectroscopy. RSC Publishing, Cambridge, p. 225 (2011)

${ }^{79}$ E. Stavitski, R.J. Smith, M.W. Bourassa, A.S. Acerbo, G.L. Carr, L.M. Miller, Dynamic full-field infrared imaging with multiple synchrotron beams Anal. Chem. 85, 3599 (2013)

${ }^{80}$ W.R. Waldman, M.A. De Paoli, Photodegradation of polypropylene/polystyrene blends: Styrene-butadiene-styrene compatibilisation effect, Polym. Degrad. Stab., 93, 273-280 (2008).

${ }^{81}$ M.J. Nasse, M.J. Walsh, E.C. Mattson, R. Reininger, A. Kajdacsy-Balla, V. Macias, R. Bhargava, C.J. Hirschmugl, High-resolution Fourier transform infrared chemical imaging with multiple synchrotron beams, Nature Methods, 8, 413-418 (2011)

${ }^{82}$ M. Unger, E. Mattson, C. Schmidt Patterson, Z. Alavi, D. Carson, C. J. Hirschmugl, Synchrotron-based multiple-beam FTIR chemical imaging of a multi-layered polymer in transmission and reflection: toward cultural heritage applications, Appl. Phys. A, 111, 135-145 (2013)

${ }^{83}$ C. Clemons, J. Sedlmair, B. Illman, R. Ibach, C. Hirschmugl, Chemically imaging the effects of the addition of nanofibrillated cellulose on the distribution of poly(acrylic acid) in poly(vinyl alcohol), Polymer 54, 2058-2061 (2013)

${ }^{84}$ M. C. Martin, C. Dabat-Blondeau, M. Unger, J. Sedlmair, D. Y. Parkinson, H. A. Bechtel, B. Illman, J. M. Castro, M. Keiluweit, D. Buschke, B. Ogle, M. J. Nasse, C. J. Hirschmugl, Nature Methods, 10, 861-864 (2013)

${ }^{85}$ M. Unger, J. Sedlmair, H.W. Siesler, C. Hirschmugl, 3D FT-IR imaging spectroscopy of phaseseparation in a poly(3-hydroxybutyrate)/poly(I-lactic acid) blend, Vibr. Spectrosc., 75, 169-172 (2014)

${ }^{86}$ S.G. Kazarian, K.L.A. Chan, F.H. Tay, ATR-FT-IR Imaging for Pharmaceutical and Polymeric Materials: From Micro to Macro Approaches, in R. Salzer and H.W. Siesler (Eds.) "Infrared and Raman Spectroscopic Imaging”, Chapter 10, pp.347-374 Wiley-VCH Verlag GmbH \& Co. KGaA, Weinheim (2009)

${ }^{87}$ S.G. Kazarian, K.L.A. Chan. "Chemical Photography" of Drug Release. Macromolecules, 37, 9866-9872 (2003)

${ }^{88}$ C. Keles, A. Naylor, F. Clegg, C. Sammon, Studying the release of hGH from gamma-irradiated PLGA microparticles using ATR-FTIR imaging, Vibr. Spectrosc. 71, 76-84 (2014)

${ }^{89}$ C. Keles, A. Naylor, F. Clegg, C. Sammon, Investigation of factors influencing the hydrolytic degradation of single PLGA microparticles, Polym. Degrad. Stab., 119, 228-241 (2015)

${ }^{90}$ A. J. Sommer, L. G. Tisinger, C. Marcott, G. M. Story. Attenuated Total Internal Reflection Infrared Mapping Microspectroscopy Using an Imaging Microscope. Appl. Spectrosc., 55, 252256 (2001)

${ }^{91}$ S.G. Kazarian, K.L.A. Chan. ATR-FTIR spectroscopic imaging: recent advances and applications to biological systems, Analyst, 138, 1940 (2013)

92 K.L.A. Chan, S. Gulati, J. B. Edel, A. J. de Mello, S.G. Kazarian, Chemical imaging of microfluidic flows using ATR-FTIR spectroscopy, Lab Chip, 9, 2909-2913 (2009)

${ }^{93}$ K.L.A. Chan, S.G. Kazarian. FT-IR Spectroscopic Imaging of Reactions in Multiphase Flow in Microfluidic Channels, Anal. Chem., 84, 4052-4056 (2013)

${ }^{94}$ Z. Wang, D. Voicu, L. Tang, W. Li, E. Kumacheva, Microfluidic studies of polymer adsorption in flow, Lab Chip, 15, 2110 (2015)

${ }_{95}$ M.K. Kuimova, K.L.A. Chan, S.G. Kazarian, Chemical imaging of live cancer cells in the natural aqueous environment. Appl. Spectrosc., 63, 164-171 (2009)

${ }^{96}$ a) H.-Y. N. Holman, R. Miles, Z. Hao, E. Wozei, L. M. Anderson, H. Yang, Real-Time Chemical Imaging of Bacterial Activity in Biofilms Using Open-Channel Microfluidics and Synchrotron FTIR Spectromicroscopy, Anal. Chem., 81, 8564-8570 (2009) b) M.J. Tobin, L. Puskar, R. L. Barber, E.C. Harveyb, P. Heraud, B. R. Wood, K. R. Bambery, C. T. Dillon, K. L. Munro, FTIR spectroscopy of single live cells in aqueous media by synchrotron IR microscopy using microfabricated sample holders, Vibr. Spectrosc., 53, 34-38 (2010); c) L. Vaccari, G. Birarda, G. Grenci, S. Pacor, L. Businaro, Synchrotron radiation infrared microspectroscopy of single living cells in microfluidic devices: advantages, disadvantages and future perspectives, J. Phys.: Conf. 
Ser. 359, 012007 (2012); d) C. Sandt, F. Jamme, P. Dumas, Profiling pluripotent stem cells and organelles using synchrotron radiation infrared microspectroscopy, J. Biophotonics, 6, 60 (2013); e) P. Gelfand, R.J. Smith, E. Stavitski, D.R. Borchelt, L.M. Miller, Characterization of Protein Structural Changes in Living Cells Using Time-Lapsed FTIR Imaging, Anal. Chem., 87, 6025-6031 (2015)

${ }^{97}$ D. Dendukuri, P. S. Doyle, The Synthesis and Assembly of Polymeric Microparticles Using Microfluidics, Adv. Mater., 21, 1-16 (2009)

${ }^{98}$ A.K. Yadav, M. Krell, W.D. Hergeth, J.C. de la Cal, M. J. Barandiaran, Monitoring Polymerization Kinetics in Microreactors by Confocal Raman Microscopy, Macromol. React. Eng., 8, 543-549 (2014)

${ }^{99}$ E. Gross, X.Z. Shu, S. Alayoglu, H. A. Bechtel, M. C. Martin, F. D. Toste, G.A. Somorjai, In Situ IR and X- ray High Spatial-Resolution Microspectroscopy Measurements of Multistep Organic Transformation in Flow Microreactor Catalyzed by Au Nanoclusters, J. Am. Chem. Soc., 136, 3624-3629 (2014)

100 a) T. Taubner, R. Hillenbrand, F. Keilmann, Performance of visible and mid-infrared scattering-type near-field optical microscopes, J. Microscopy, 210, 311-314 (2003); F. Keilmann, R. Hillenbrand, Phil. Trans.: Mat. , Phys. Eng. Sci., 362, 787-805 (2004) ${ }^{101}$ F. Huth, A. Govyadinov, S. Amarie, W. Nuansing, F. Keilmann, R. Hillenbrand, Nano-FTIR Absorption Spectroscopy of Molecular Fingerprints at $20 \mathrm{~nm}$ Spatial Resolution, Nano Lett., 12, 3973-3978 (2012)

${ }^{102}$ H.A. Bechtel, E. A. Muller, R. L. Olmon, M.C. Martin, M. B. Raschke, Ultrabroadband infrared nanospectroscopic imaging, Proc. Nat. Acad. Sci., 111, 7191-7196 (2014)

${ }^{103}$ a) L. Bozec, A. Hammiche, M. J. Tobin, J. M. Chalmers, N. J. Everall, H. M. Pollock, Near-field photothermal Fourier transform infrared spectroscopy using synchrotron radiation, Meas. Sci. Technol. 13 (2002) 1217-1222; b) B. Lahiri, G.Holland , A. Centrone, Chemical Imaging Beyond the Diffraction Limit: Experimental Validation of the PTIR Technique, Small, 9, 439-445 (2013); c) A. M. Katzenmeyer, G. Holland, J. Chae, A. Band, K. Kjoller and A. Centrone, Mid-infrared spectroscopy beyond the diffractionlimit via direct measurement of the photothermal effect, Nanoscale, 7, 17637 (2015)

${ }^{104}$ P.M. Donaldson, C.S. Kelley, M.D. Frogley, J. Filik, K. Wehbe, G. Cinque, Broadband nearfield infrared spectromicroscopy using photothermal probes and synchrotron radiation, Opt. Express, 24, 1852-1864 (2016)

${ }^{105}$ B. Pollard, F. C. B. Maia, M. B. Raschke, R. O. Freitas, Infrared Vibrational Nanospectroscopy by Self-Referenced Interferometry, Nano Lett., 16, 55-61 (2016)

${ }^{106}$ B. Pollard, E. A. Muller, K. Hinrichs, M. B. Raschke, Vibrational nano-spectroscopic imaging correlating structure with intermolecular coupling and dynamics, Nature Comm., 5, 3587 (2014)

${ }^{107}$ M. Abo-Bakr, J. Feikes, K. Holldack, P. Kuske, W.B Peatman, U. Schade, G. Wüstefeld, H.-W. Hübers, Brilliant, coherent far-infrared (THz) synchrotron radiation, Phys. Rev. Lett., 90, 09481 (2003)

${ }^{108}$ U. Schade, K. Holldack, P. Kuske, G. Wüstefeld, H.-W. Hübers, THz near-field imaging employing synchrotron radiation, Appl. Phys. Lett., 84, 1422-1424 (2004)

${ }^{109}$ M. Mrosko, M. Koch-Muller, U. Schade, In-situ mid/far micro-FTIR spectroscopy to trace pressure-induced phase transitions in strontium feldspar and wadsleyite, Amer. Minerol., 96, 1748-1759 (2011)

${ }^{110}$ Y.-S. Jin, G.-J. Kim, S. -G. Jeon, Terahertz Dielectric Properties of Polymers, J. Korean Phys. Soc., 49, 513-517 (2006)

${ }^{111}$ P.D. Cunningham, N.N. Valdes, F. A. Vallejo, M. Hayden, Brent Polishak, X.-H. Zhou, J. Luo, A.K.-Y. Jen, J. C. Williams, R. J. Twieg, Broadband terahertz characterization of the refractive index and absorption of some important polymeric and organic electro-optic materials, J. Appl. Phys., 109, 044505 (2011)

${ }^{112}$ S.Wietzke, C. Jansen, T. Jung, M. Reuter, B. Baudrit, M. Bastian, S. Chatterjee, M.Koch, Terahertz time-domain spectroscopy as a tool to monitor the glass transition in polymers, Opt. Express, 17, 19006-19014 (2009) 
${ }^{113}$ P. Loubeyre, F. Occelli, P. Dumas. Hydrogen phase IV revisited via synchrotron infrared measurements in $\mathrm{H}_{2}$ and $\mathrm{D}_{2}$ up to $290 \mathrm{GPa}$ at $296 \mathrm{~K}$, Phys. Rev. B, 87, 134101 (2013)

${ }^{114}$ V. Schettino, R. Bini, M. Ceppatelli, L. Ciabini, M. Citroni, Chemical reactions at very high pressure, in Advances in Chemical Physics, (Ed. S.A. Rice), Vol. 131, pp. 105-242, Wiley, New York (2005); R. Bini, V. Schettino, Materials Under Extreme Conditions, Molecular Crystals at High Pressure, Imperial College Press: London (2014)

115 a) J.R. Schoonover, D.M. Dattelbaum, J.C. Osborn, J.S. Bridgewater, J.W. Kenney III, Pressure-dependent Fourier transform infrared spectroscopy of a poly (ester urethane), Spectrochim. Acta Part A, 59, 309-319 (2003); b) E. D. Emmons, R. G. Kraus, S. S. Duvvuri, J. S. Thompson, A. M. Covington, High-Pressure Infrared Absorption Spectroscopy of Poly(Methyl Methacrylate), J. Polym. Sci.: Part B: Polym. Phys. 45, 358-367 (2007)

${ }^{116}$ D. Cavallo, Univ. Genova, Italy. Personal Communication

117 a) A.L. Kovarskii, "High-Pressure Chemistry and Physics of Polymers" CRC Press, 1994 ISBN: 0849342392; b) M. Sakashita, H. Yamawaki, K. Aoki, FT-IR Study of the Solid State Polymerization of Acetylene under Pressure, J. Phys. Chem., 100, 9943-9947 (1996)

${ }^{118}$ M. Ceppatelli , R. Bini, Light-Induced Catalyst and Solvent-Free High Pressure Synthesis of High Density Polyethylene at Ambient Temperature. Macromol. Rapid Commun., 35, 787-793 (2014)

${ }^{119}$ D. Scelta, M. Ceppatelli, M. Santoro, R. Bini, F.A. Gorelli, A. Perucchi, M. Mezouar, A. van der Lee, J. Haines, High Pressure Polymerization in a Confined Space: Conjugated Chain/Zeolite Nanocomposites. Chem. Mater., 26, 2249-2255 (2014) 


\begin{tabular}{|c|c|c|c|c|c|c|c|c|c|c|}
\hline \multirow{3}{*}{ Facility } & \multirow{3}{*}{ Location } & \multirow{3}{*}{ Status ${ }^{a}$} & \multicolumn{8}{|c|}{ SR Techniques available ${ }^{b}$} \\
\hline & & & \multirow{2}{*}{ Micr } & \multirow{2}{*}{ FIR } & \multicolumn{3}{|c|}{ Imaging } & \multirow{2}{*}{ HP } & \multirow{2}{*}{ EL } & \multirow{2}{*}{ MF } \\
\hline & & & & & ATR & $\mathbf{F F}$ & Nano & & & \\
\hline \multicolumn{11}{|c|}{ Americas } \\
\hline ALS & Lawrence Berkeley Nat. Lab., CA, USA & 0 & 3 & 2 & & & & & & \\
\hline CAMD & Louisiana State Univ., LA, USA & $\mathrm{O}$ & & & & & & & & \\
\hline CLS & Saskatoon, CANADA & $\mathrm{O}$ & & & & & & & & \\
\hline LNLS & Campinas, BRASIL & 0 & & & & & & & & \\
\hline $\mathrm{NSLS}^{\mathrm{C}}$ & Brookhaven Natl. Lab., NY, USA & $D^{c}$ & 4 & 2 & & & & & & \\
\hline$S_{R C^{d}}$ & Wisconsin, USA & $D^{d}$ & & & & & & & & \\
\hline \multicolumn{11}{|c|}{ Europe \& North Africa } \\
\hline ALBA $^{\mathrm{e}}$ & Barcelona, SPAIN & $\mathrm{C}^{\mathrm{e}}$ & & & & & & & & \\
\hline ANKA & Karlsruhe, GERMANY & 0 & & & & & & & & \\
\hline ASTRID & Aarhus, DENMARK & $\mathrm{C}$ & & & & & & & & \\
\hline BESSY II & HZB, Berlin, GERMANY & $\mathrm{O}$ & & & & & & & & \\
\hline DAFNE & Frascati, ITALY & 0 & & & & & & & & \\
\hline DELTA & Dortmund, GERMANY & $\mathrm{P}$ & & & & & & & & \\
\hline DIAMOND & Didcot, Oxon, UK & $\mathrm{O}$ & 2 & & & & & & & \\
\hline ELETTRA & Trieste, ITALY & $\mathrm{O}$ & & & & & & & & \\
\hline ESRF & Grenoble, FRANCE & $\mathrm{O}$ & & & & & & & & \\
\hline MAX LAB & Lund, SWEDEN & 0 & & 2 & & & & & & \\
\hline SESAME & JORDAN & $\mathrm{P}$ & & & & & & & & \\
\hline MLS & Berlin, GERMANY & 0 & & & & & & & & \\
\hline SLS & Villigen, SWITZERLAND & $\mathrm{D}$ & & & & & & & & \\
\hline SOLEIL & Paris, FRANCE & 0 & 2 & 2 & & & & & & \\
\hline \multicolumn{11}{|c|}{ Asia \& Australia } \\
\hline AS & Melbourne, AUSTRALIA & $\mathrm{O}$ & & & & & & & & \\
\hline BSRF & Beijing, CHINA & $\mathrm{P}$ & & & & & & & & \\
\hline HELIOS II & National Univ. Singapore, SINGAPORE & 0 & & & & & & & & \\
\hline INDUS I & Indore, INDIA & C & & & & & & & & \\
\hline NSRL & Hefei, CHINA & $\mathrm{P}$ & & & & & & & & \\
\hline NSRRC & Hsinchu, TAIWAN & 0 & & & & & & & & \\
\hline SLRI & Nakhon Ratchasima, THAILAND & 0 & & & & & & & & \\
\hline SPRING-8 ${ }^{\dagger}$ & Hyogo, JAPAN & 0 & & & & & & & & \\
\hline SRRF & Shanghai, CHINA & 0 & & & & & & & & \\
\hline UVSOR & Okasaki, JAPAN & 0 & & 2 & & & & & & \\
\hline
\end{tabular}

Disclaimer: The information in this table is the authors' personal vision of the state-of-the-art compiled from various sources with view to helping future users to find the techniques of interest. Since the field is developing very quickly due to increasing user demands, the panorama may be different at the time of publication. Future users are recommended to contact the facilities and beamlines directly in order to confirm the availability of specific technique.

\footnotetext{
a $\mathrm{O}=$ Operational, $\mathrm{C}=$ Under construction or commissioning, $\mathrm{P}=$ Projected, $\mathrm{D}=$ decommissioned

b KEY: $\mathbf{M i c r}=$ microspectroscopy endstation; FIR = Far infrared and /or THz beamline; ATR = Attenuated Total Reflection Mapping/Imaging; FF = full-field imaging via multiple-beam or large opening angle; Nano = near-field tip-based (sSNOM/AFM) spectroscopy and imaging; HP = high-pressure capabilities; EL = IR spectroscopic ellipsometry; $\mathbf{M F}$ = Microfluidics devices

c The NSLS closed down at the end of 2014, and the new NSLS II storage ring is in commissioning. The IR program at NSLS-II is due to restart in 2018. Two beamlines are currently envisaged.

d The Synchrotron Radiation Center (SRC) was shut down in 2014. Of interest is the in process transfer of the IRENI multi-beam IR imaging beamline to CAMD.

e First users scheduled for November 2016

${ }^{\mathrm{f}}$ At Spring-8 there is also a magneto-optical microscope (MOSS) for reflectance microspectroscopy of materials under high magnetic fields
} 
Hyperlinks (for online edition, and supporting info?).

\begin{tabular}{|c|c|}
\hline Facility & WWW \\
\hline \multicolumn{2}{|r|}{ Americas } \\
\hline ALS & http://infrared.als.lbl.gov/content/ \\
\hline CAMD & http://www.camd.lsu.edu/beamlines.htm \\
\hline CLS & http://www.lightsource.ca/beamlines/midir.php \\
\hline LNLS & http://lnls.cnpem.br/beamlines/ir1/ \\
\hline NSLS II & https://www.bnl.gov/ps/infrared/ \\
\hline \multicolumn{2}{|r|}{ Europe \& Middle East } \\
\hline ALBA & https://www.cells.es/en/beamlines \\
\hline ANKA & http://www.anka.kit.edu/968.php \\
\hline ASTRID & http://www.isa.au.dk/ \\
\hline BESSY II & $\begin{array}{l}\text { https://www.helmholtz-berlin.de/user/experimental- } \\
\text { infrastructures/instruments-photons/bessy-beamline_en.html }\end{array}$ \\
\hline DAФNE & https://web.infn.it/Dafne_Light/index.php/beamlines/sinbad-ir \\
\hline DIAMOND & $\begin{array}{l}\text { http://www.diamond.ac.uk/Beamlines/Soft-Condensed- } \\
\text { Matter/B22.html }\end{array}$ \\
\hline ELETTRA & http://www.elettra.eu/elettra-beamlines/sissi.html \\
\hline ESRF & $\begin{array}{l}\text { http://www.esrf.eu/home/UsersAndScience/Experiments/XNP } \\
\text { /ID21/SrFtir.html }\end{array}$ \\
\hline MAX LAB & https://www.maxlab.lu.se/beamlines \\
\hline MLS & https://www.ptb.de/mls/mls_beamlines.html \\
\hline SESAME & $\begin{array}{l}\text { http://www.sesame.org.jo/sesame/machine-and- } \\
\text { beamlines/beamlines.html }\end{array}$ \\
\hline SLS & https://www.psi.ch/sls/ir/ir \\
\hline SOLEIL & $\begin{array}{l}\text { http://www.synchrotron- } \\
\text { soleil.fr/Recherche/LignesLumiere/SMIS }\end{array}$ \\
\hline \multicolumn{2}{|r|}{ Asia \& Australia } \\
\hline AS & $\begin{array}{l}\text { http://www.synchrotron.org.au/aussyncbeamlines/infrared- } \\
\text { micro/introduction }\end{array}$ \\
\hline BSRF & $\begin{array}{l}\text { http://english.ihep.cas.cn/rs/fs/srl/facilityinformation/beamlin } \\
\text { eintroduction/ }\end{array}$ \\
\hline HELIOS II & http://ssls.nus.edu.sg/facility/ismi-1.html \\
\hline INDUS I & http://www.cat.ernet.in/technology/accel/indus/ \\
\hline NSRL & http://en.nsrl.ustc.edu.cn/ \\
\hline NSRRC & http://efd.nsrrc.org.tw/EFD.php?num=250 \\
\hline SLRI & http://www.slri.or.th/en/ \\
\hline SPRING-8 & $\begin{array}{l}\text { http://www.spring8.or.jp/wkg/BL43IR/instrument/lang- } \\
\text { en/INS-0000001427/instrument_summary_view }\end{array}$ \\
\hline SSRF & http://ssrf.sinap.ac.cn/english/ \\
\hline UVSOR & https://www.uvsor.ims.ac.jp/beamlines/6B/ebl6b.html \\
\hline
\end{tabular}




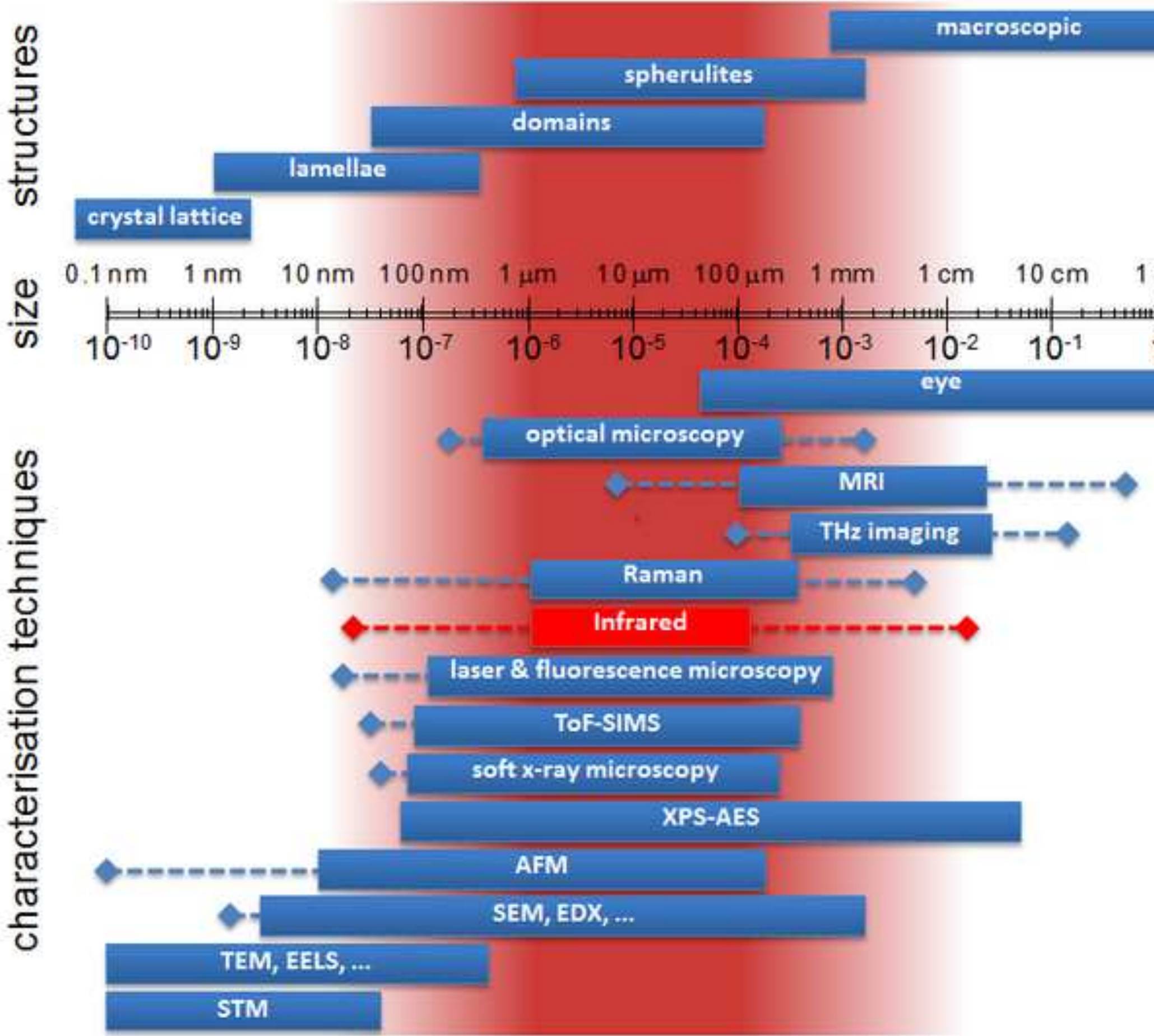




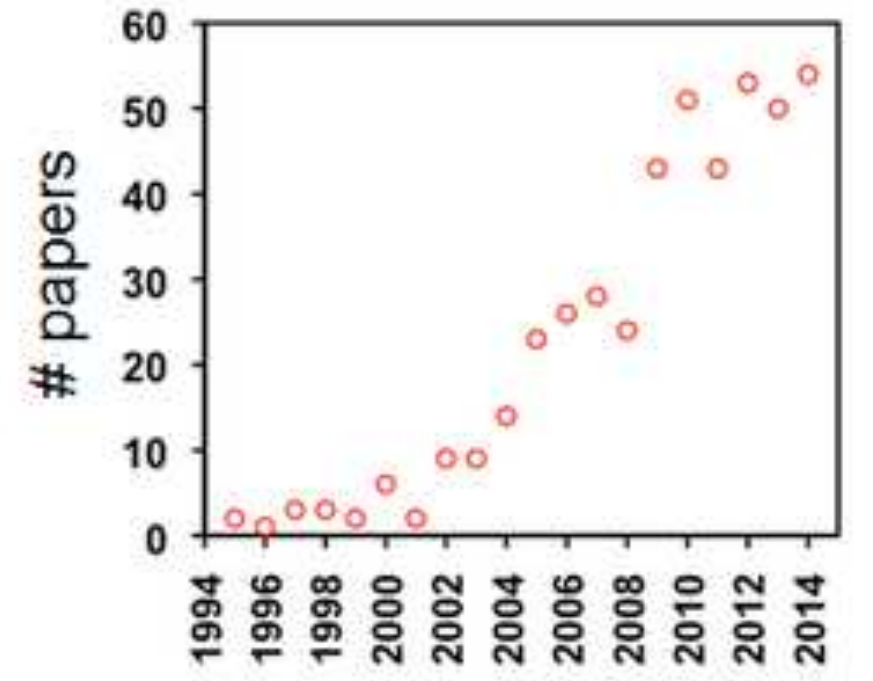

Year

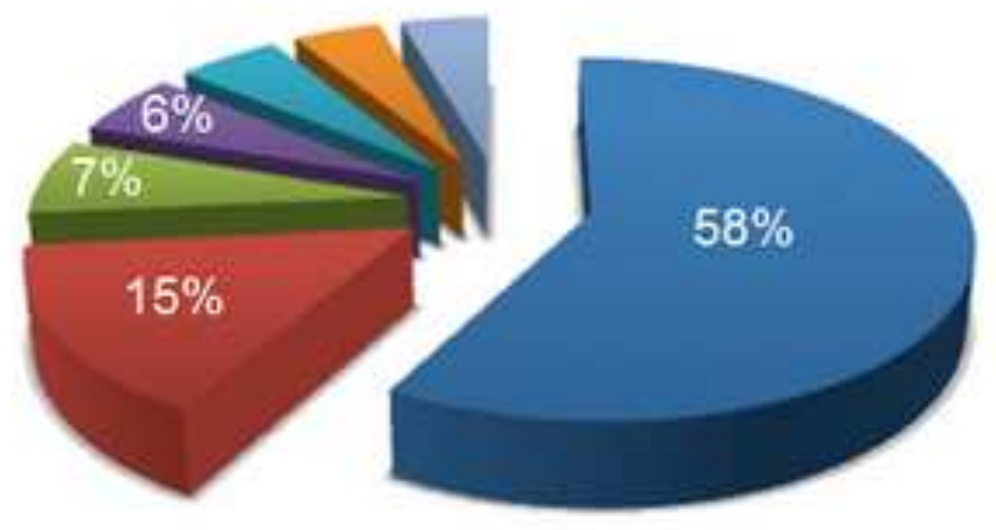

- Biology \& Life Sciences

in Instruments \& Methods

warth, Space \& Environment

Eolymers

- Cultural Heritage \& Forensics

$=$ Chemical \& Materials Sci

= Solid-state physics 


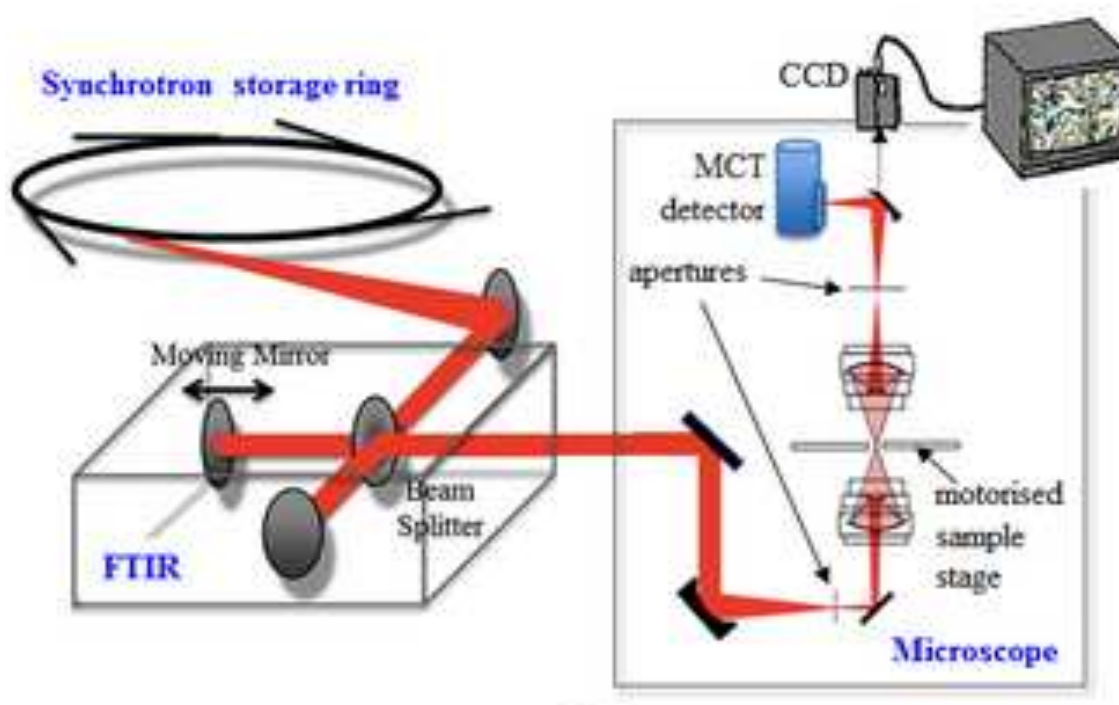

(a)
Syachrotron storage riag

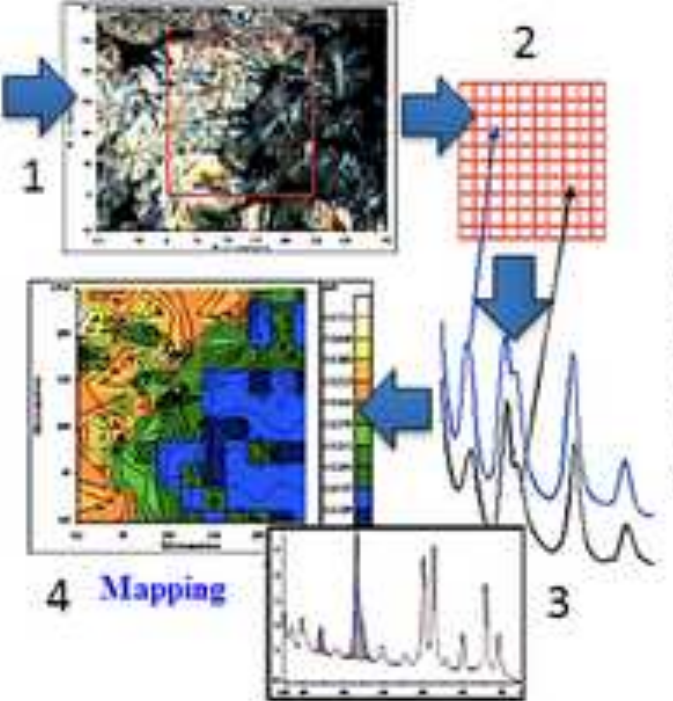

(b)

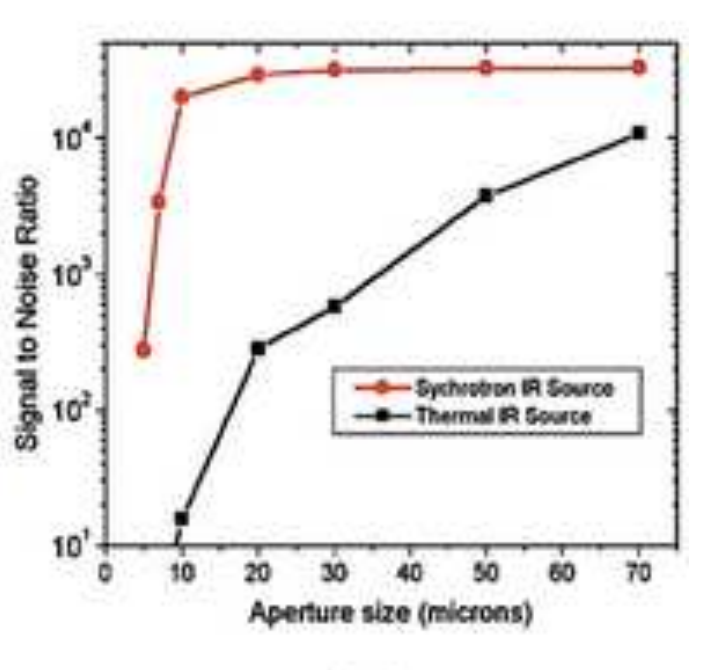

(c) 


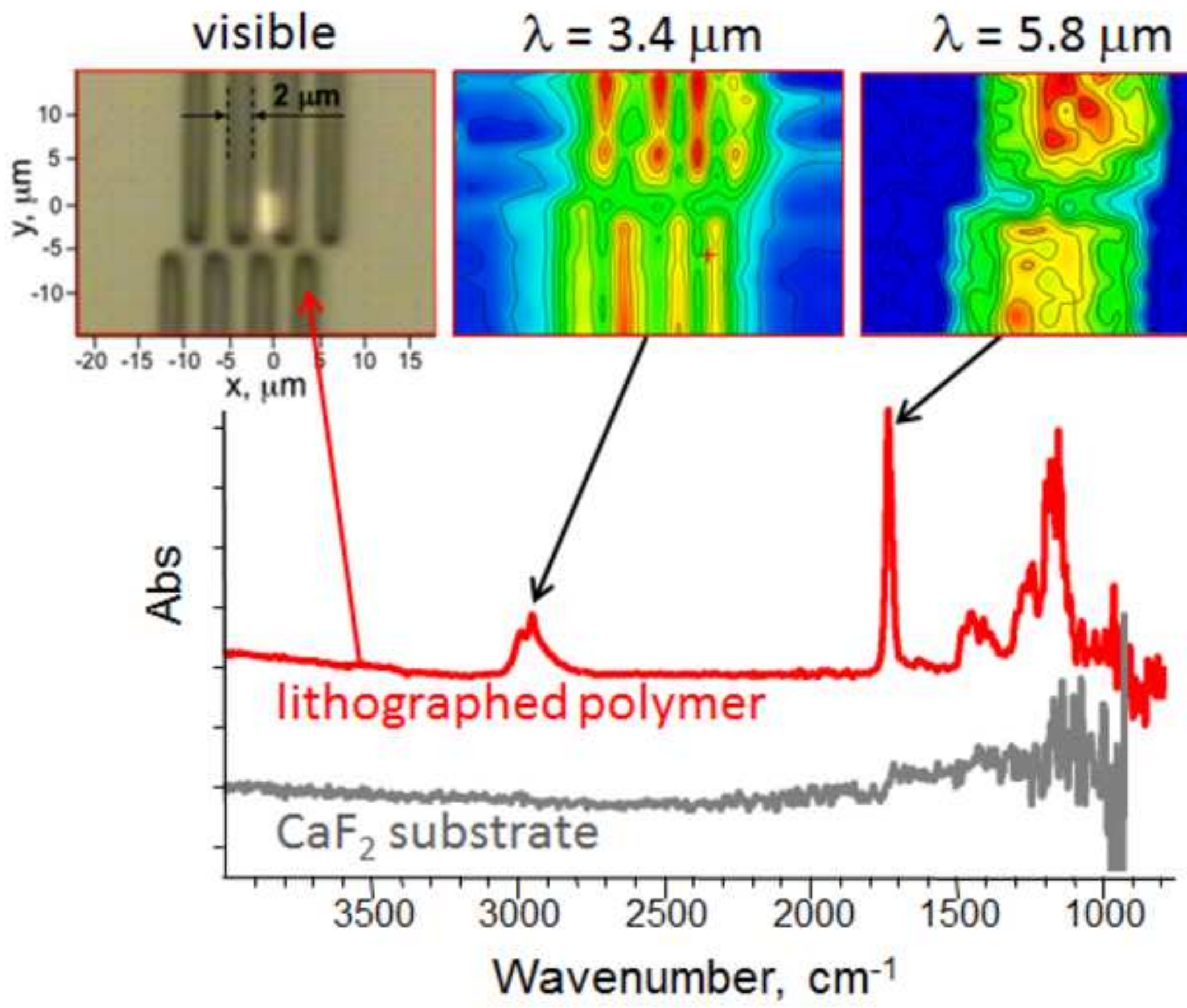


(a)

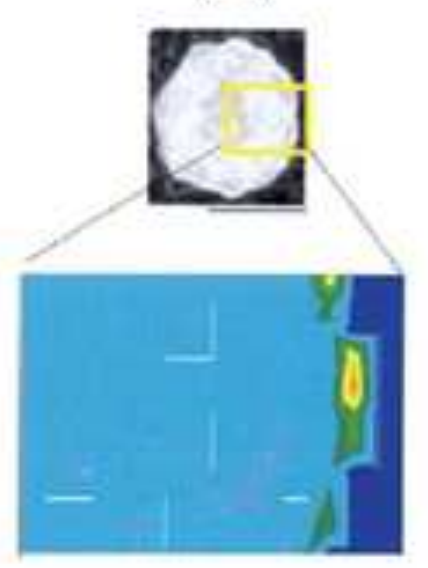

(b)

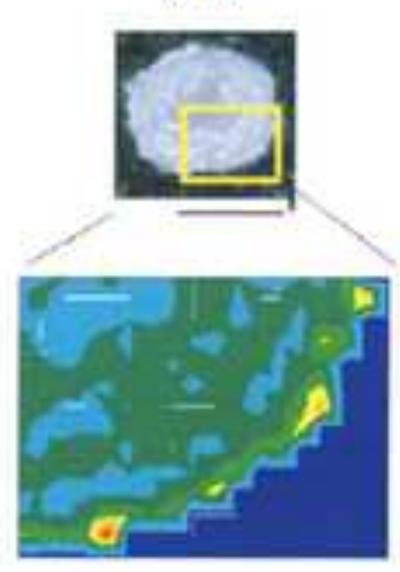

(c)

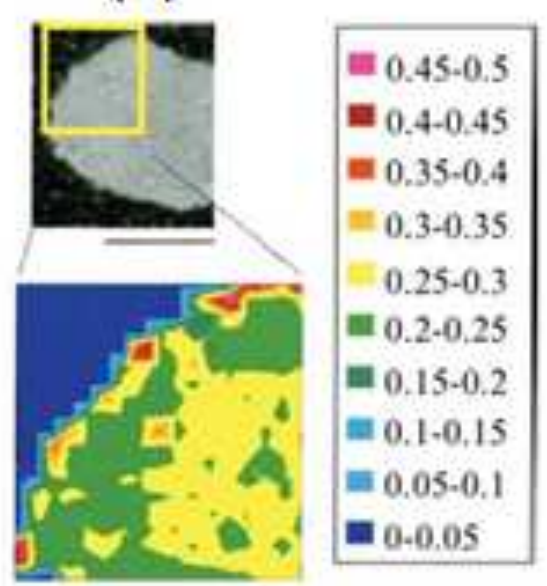

(d)

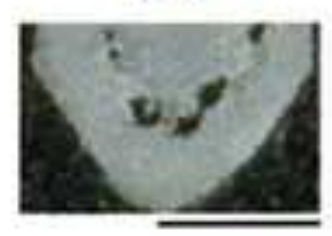

$=0.45-0.5$

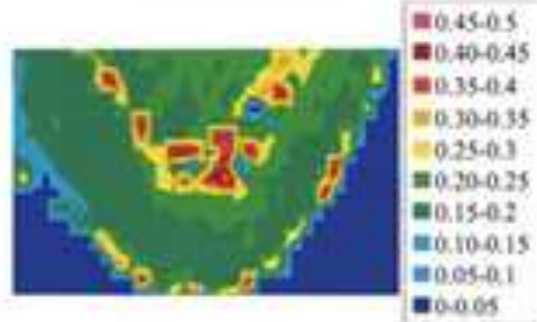

(a)

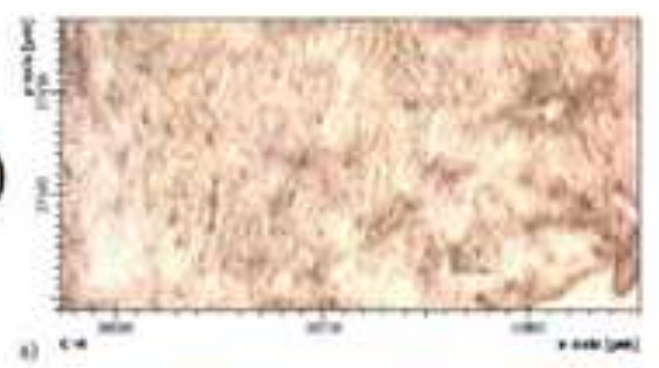

(c)

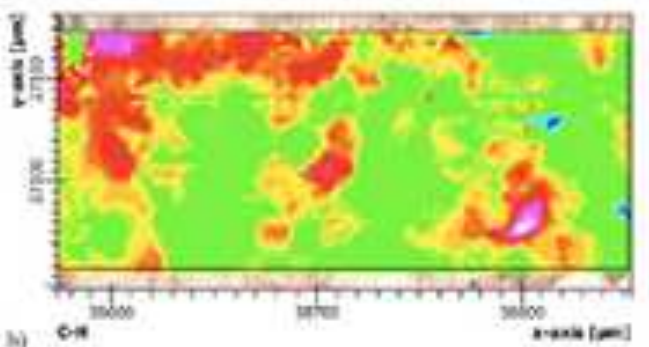

(e)

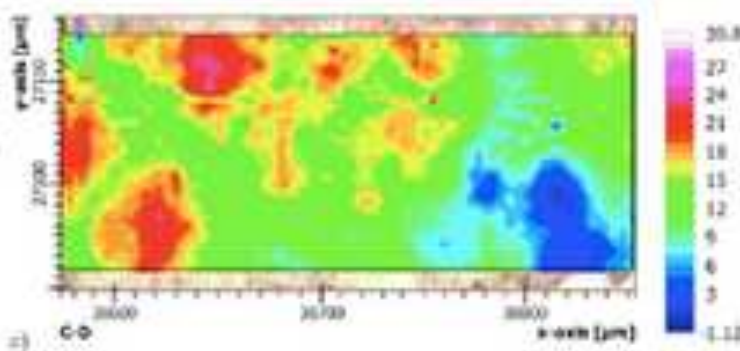

(b)

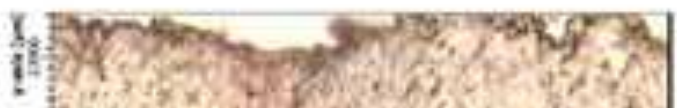

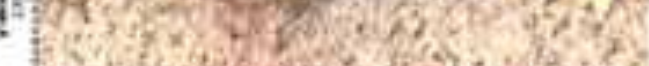

(g)

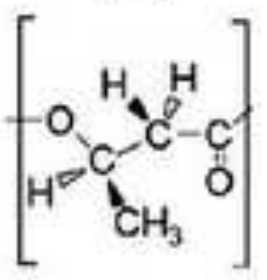

(h)

(d)

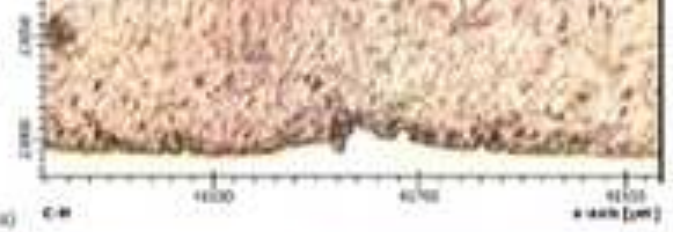

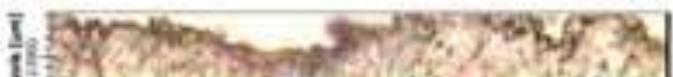
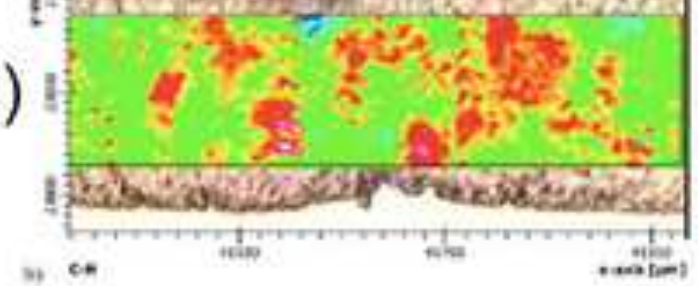

...nim,

(f)

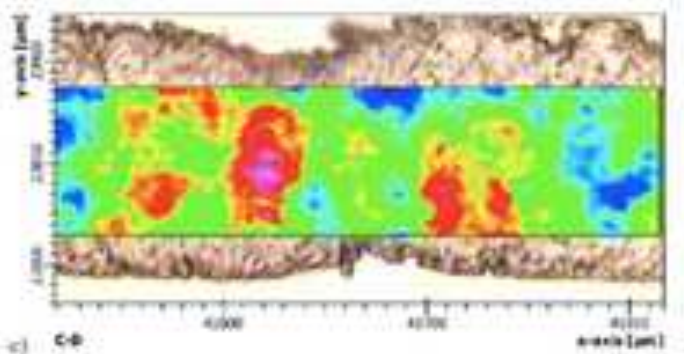

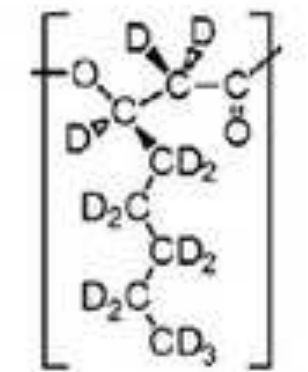



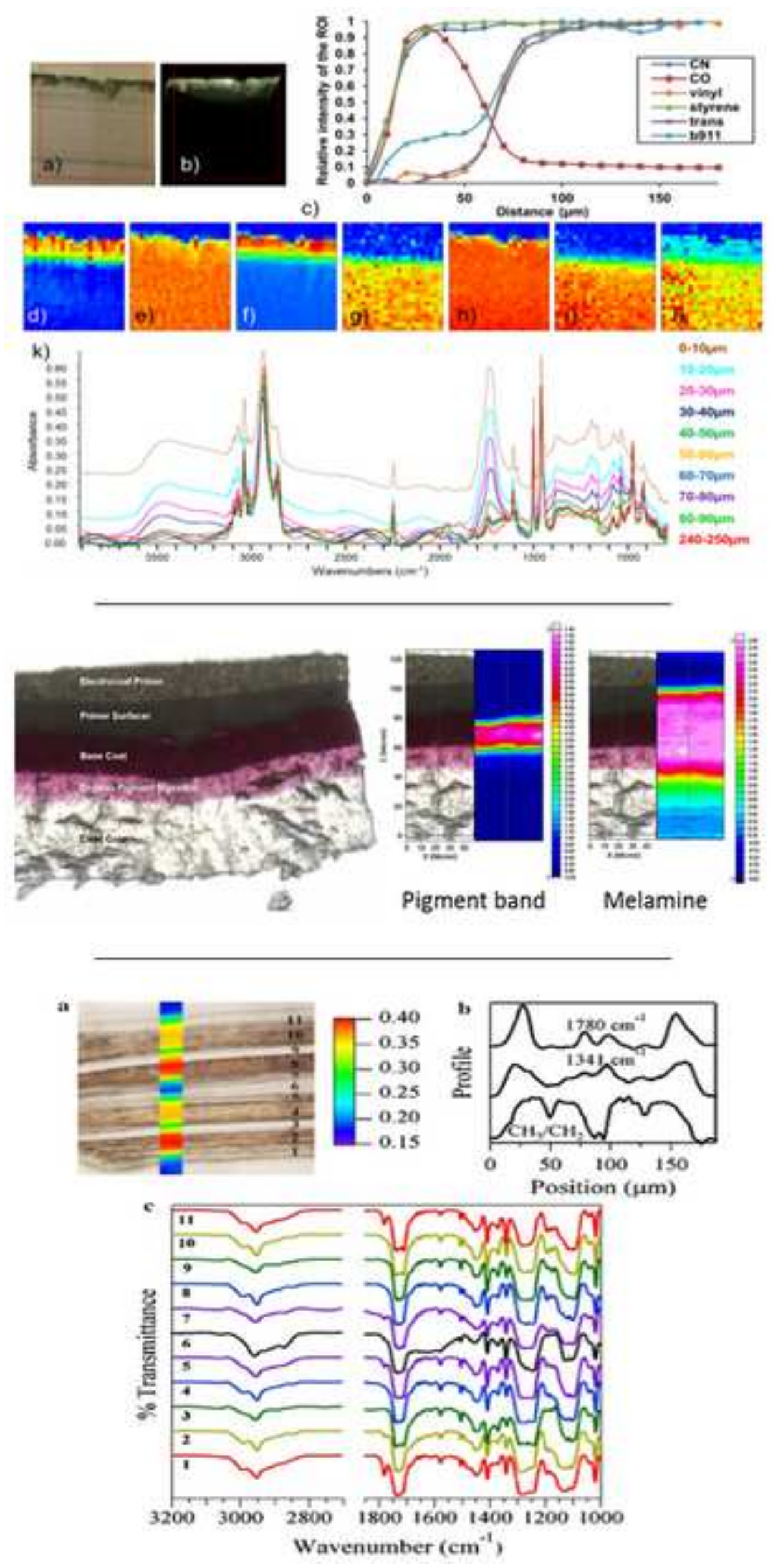

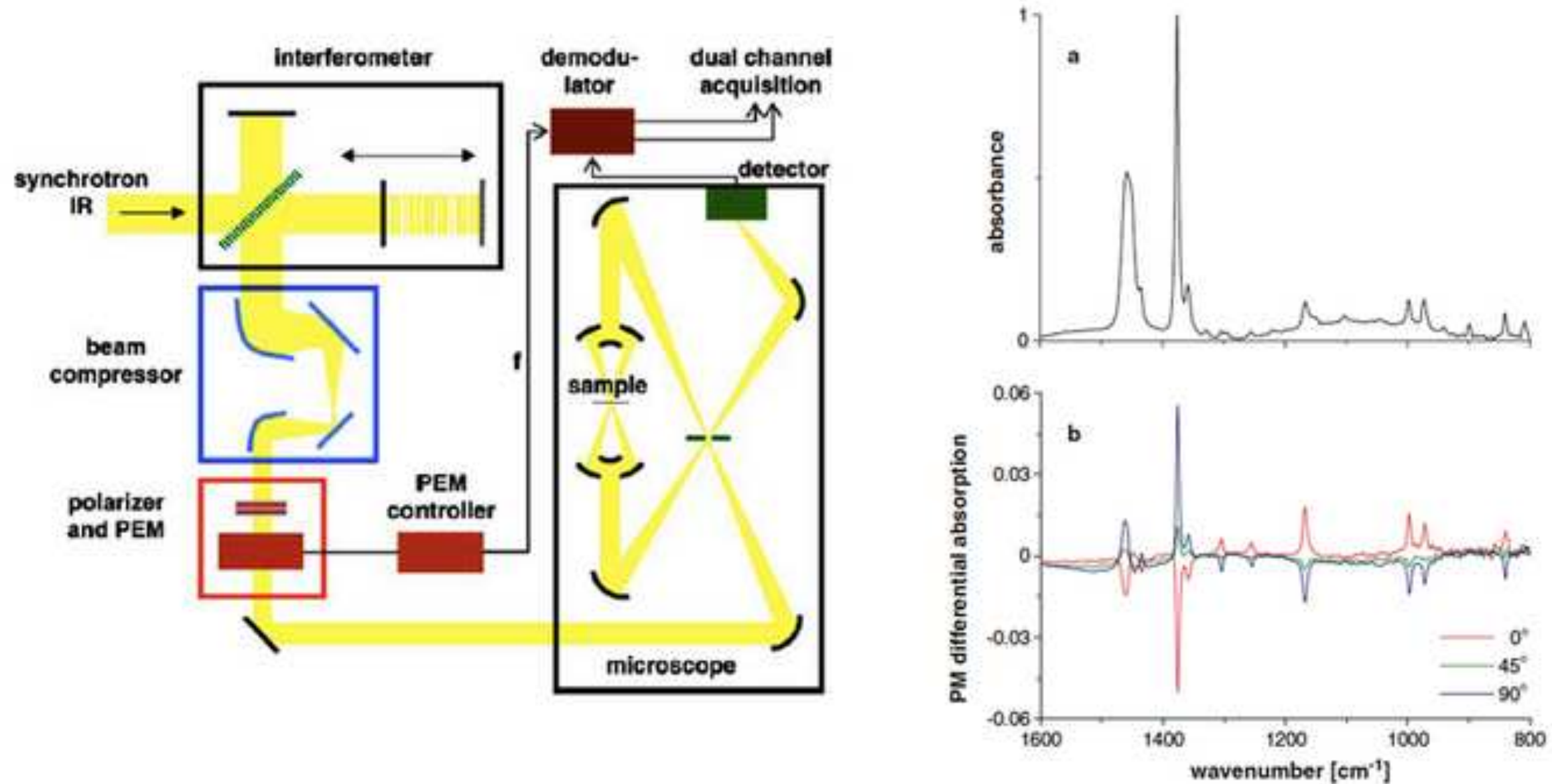
(a)

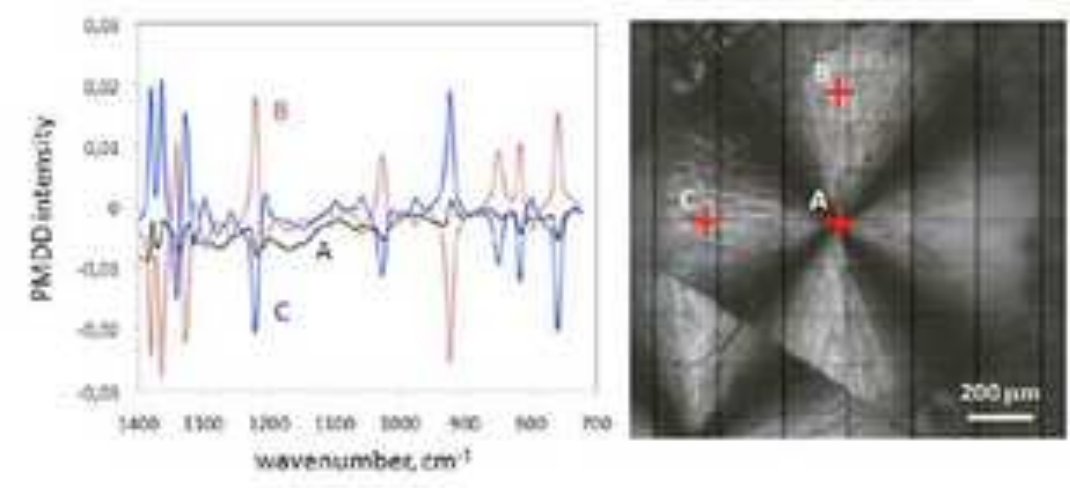

(b)

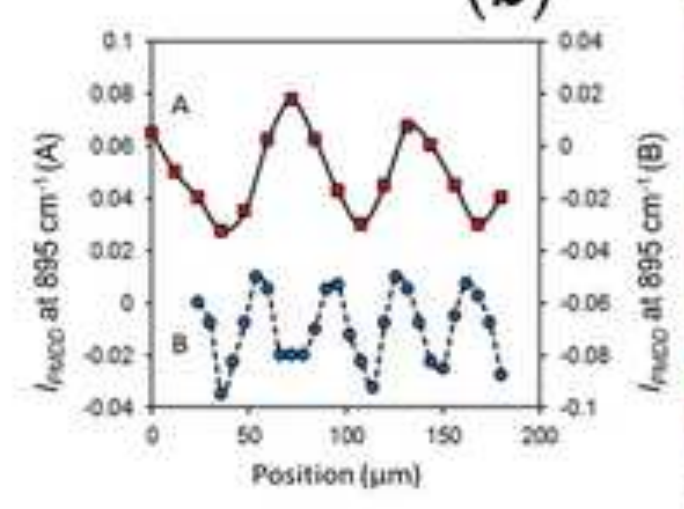

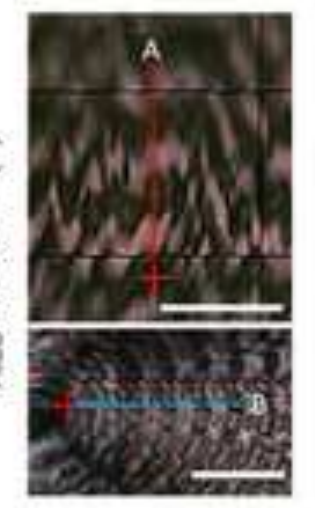

(c)

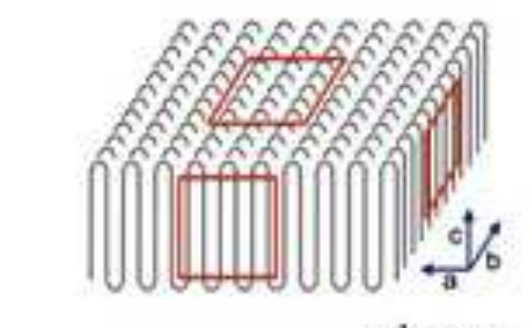

flat-on edge-on

c)

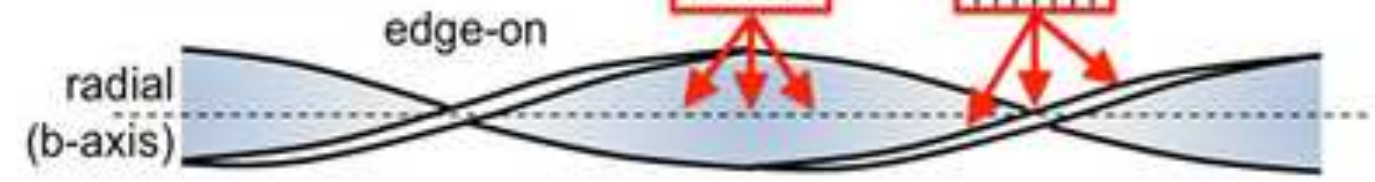

(d)

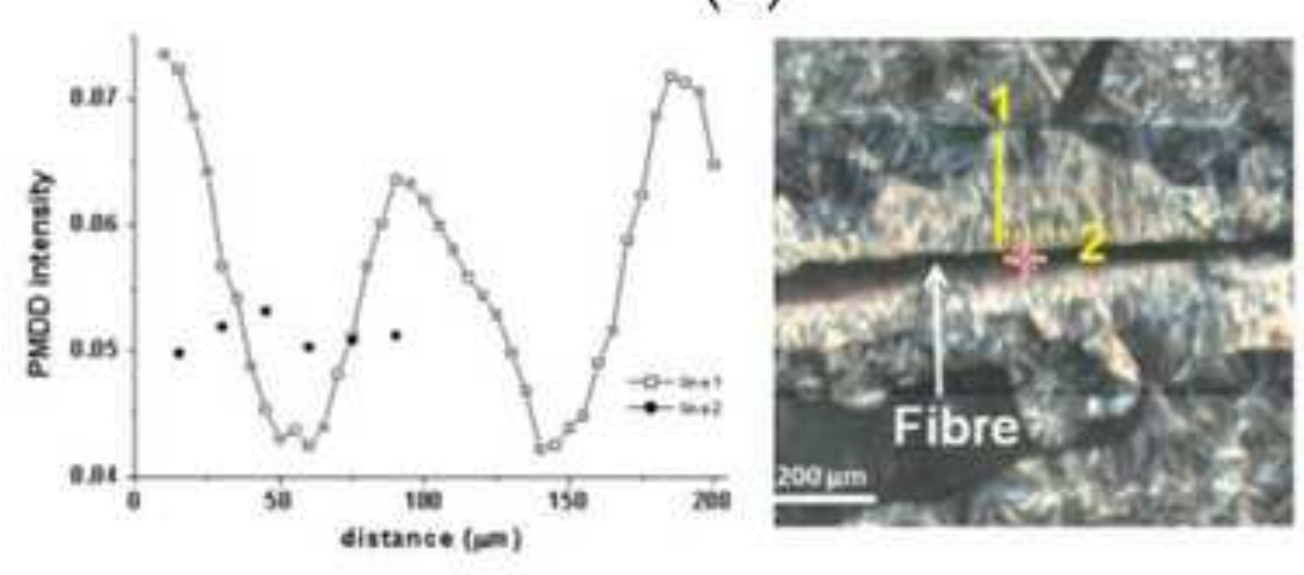

(e)

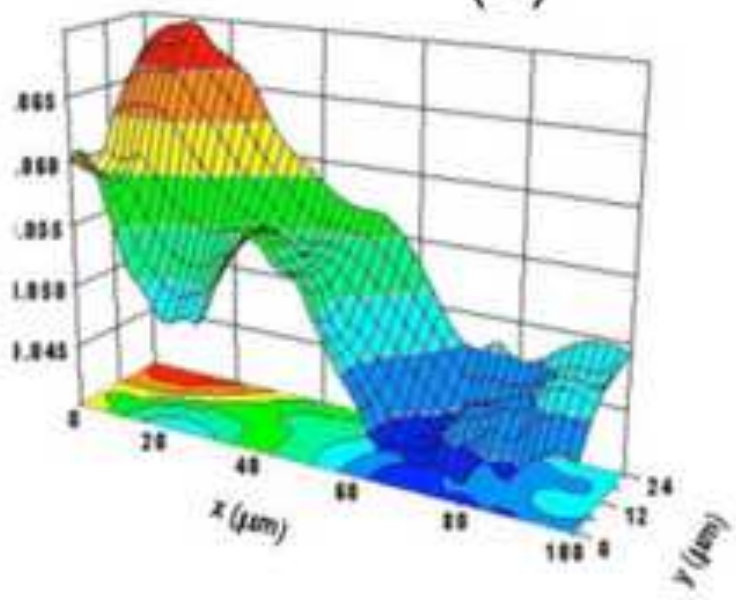


(a)
Polarizer
Analyzer

Linearly polarized

light

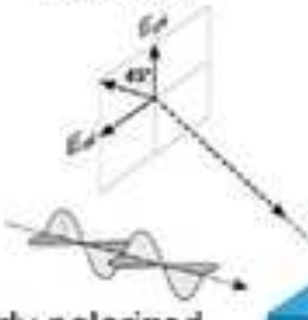

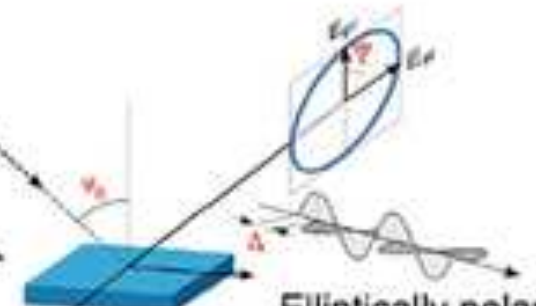
light
Elliptically polarized

(c)

(e)

$$
\frac{r_{p}}{r_{s}}=\tan \Psi e^{i \Delta}
$$

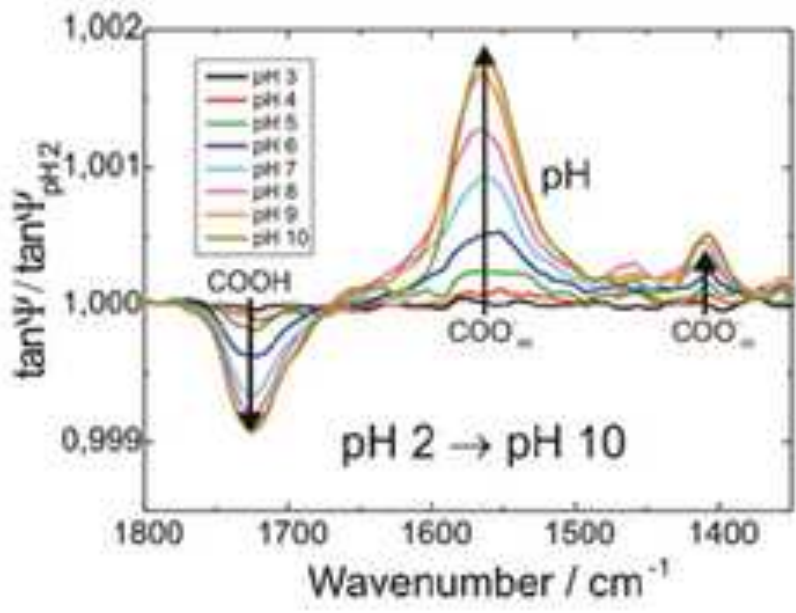

(b)

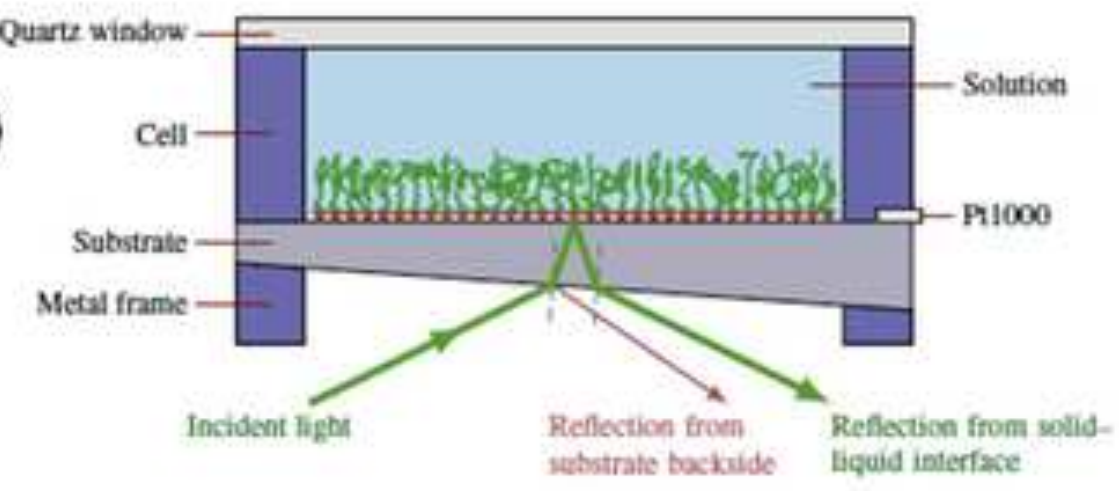

(d)

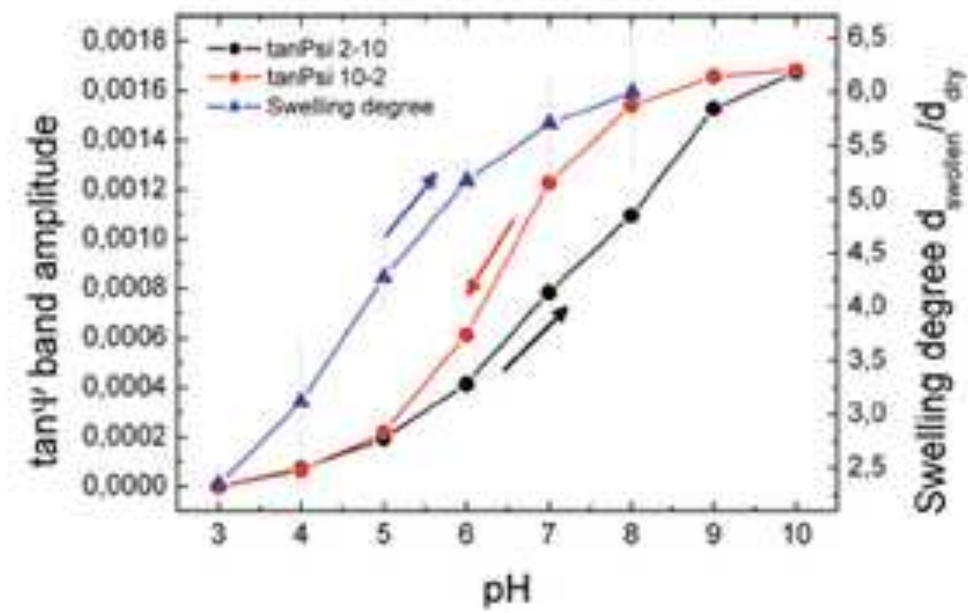

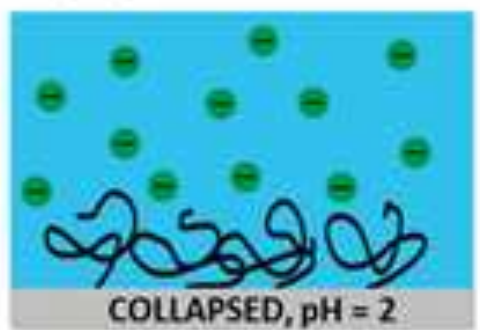

COLLAPSED, $\mathrm{pH}=2$

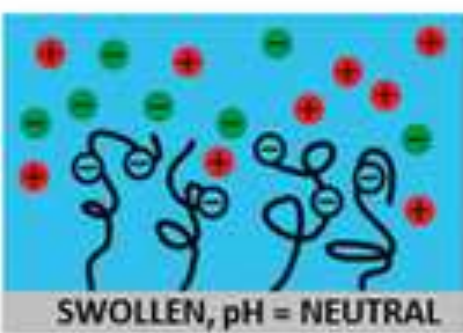

SWOLLEN, PH = NEUTRAL.

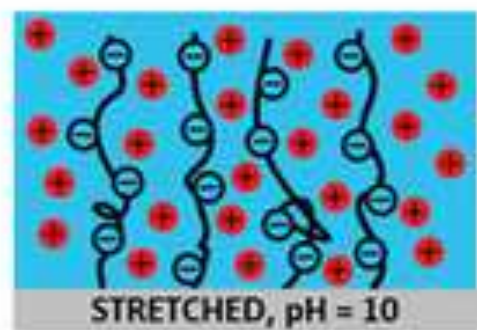

STRETCHED, $\mathrm{pH}=10$
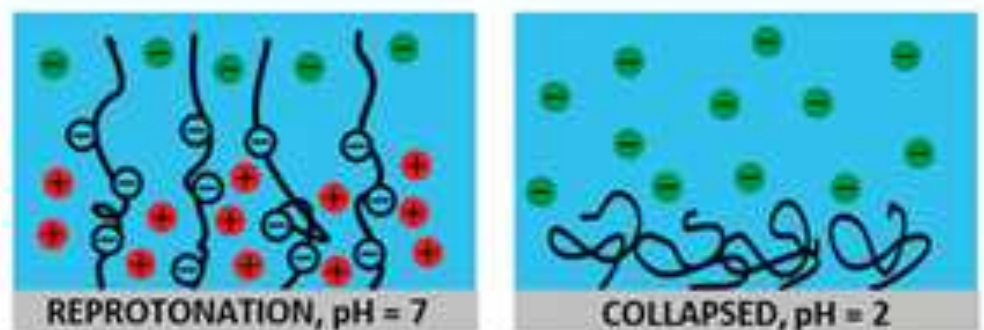
(a)

(b)

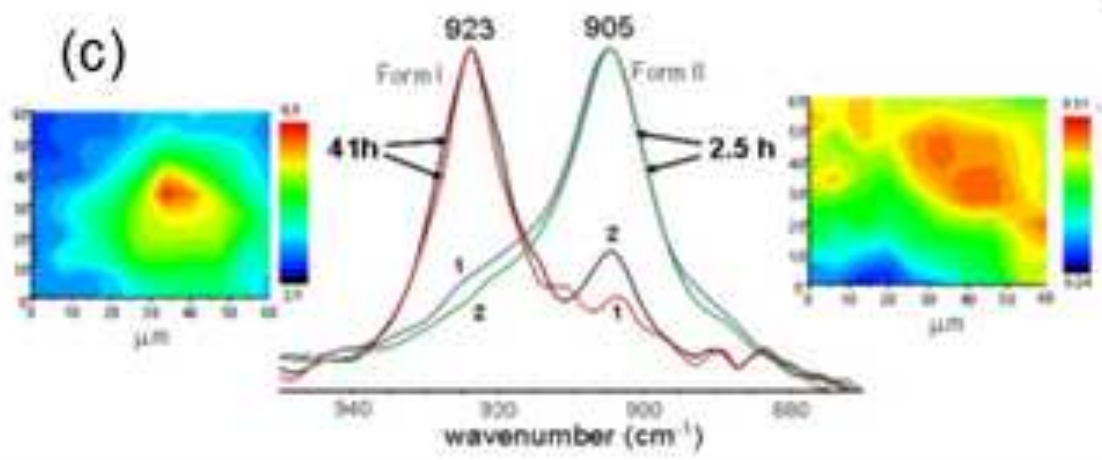

(e)

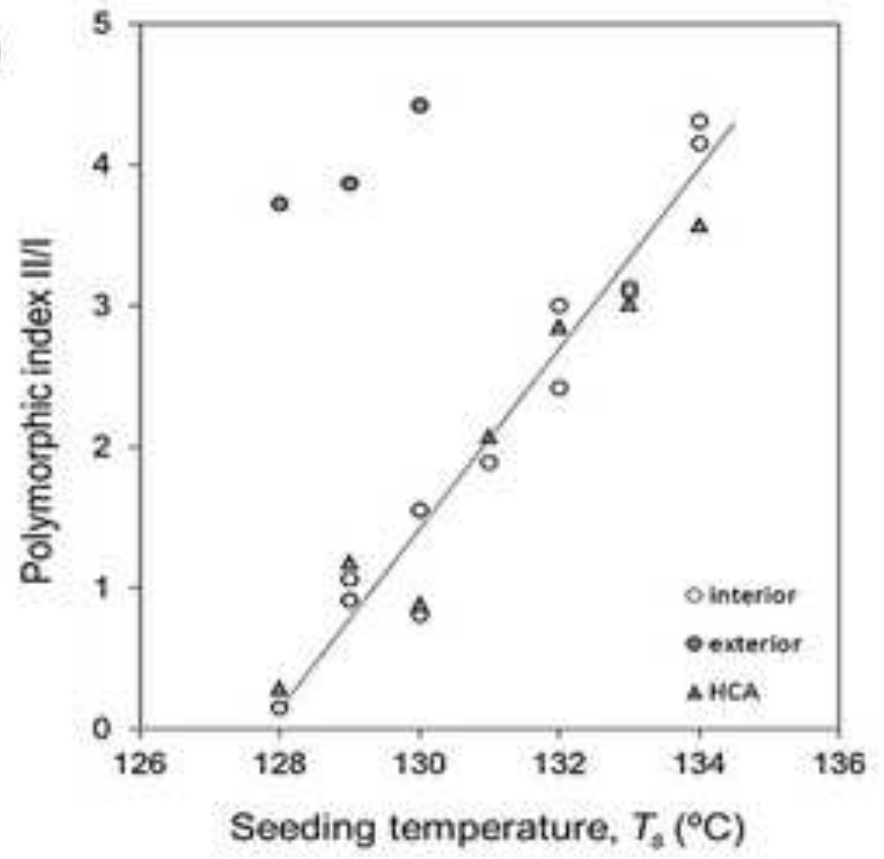

POM III images HCA images

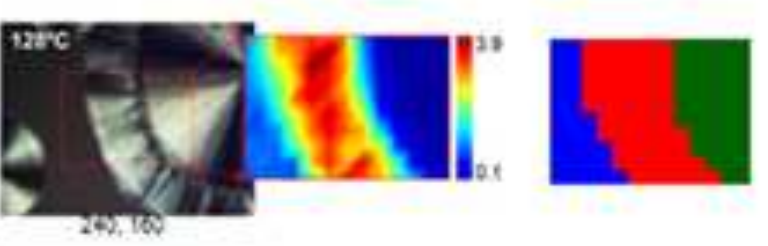

(d)
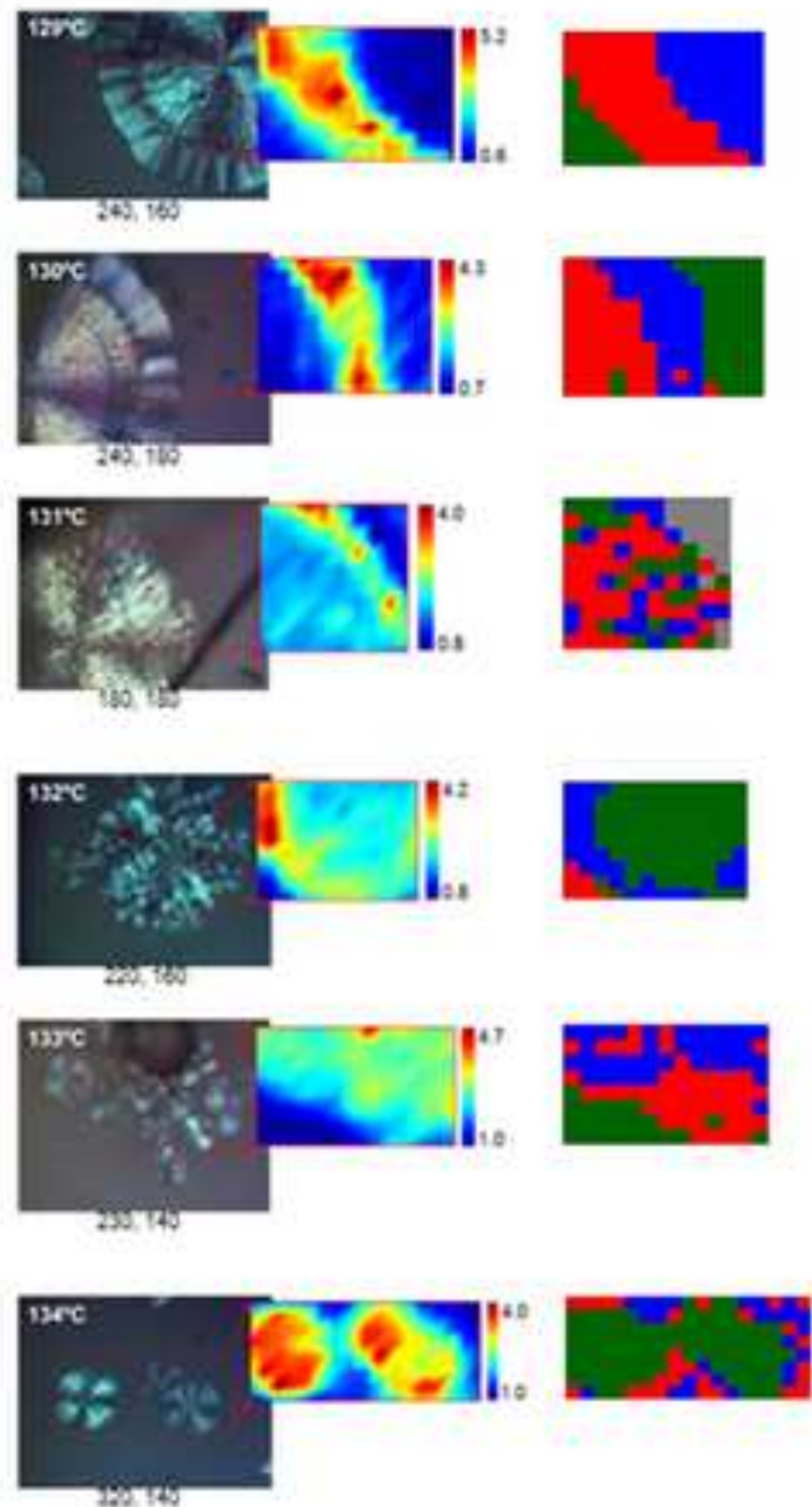
Figure 11
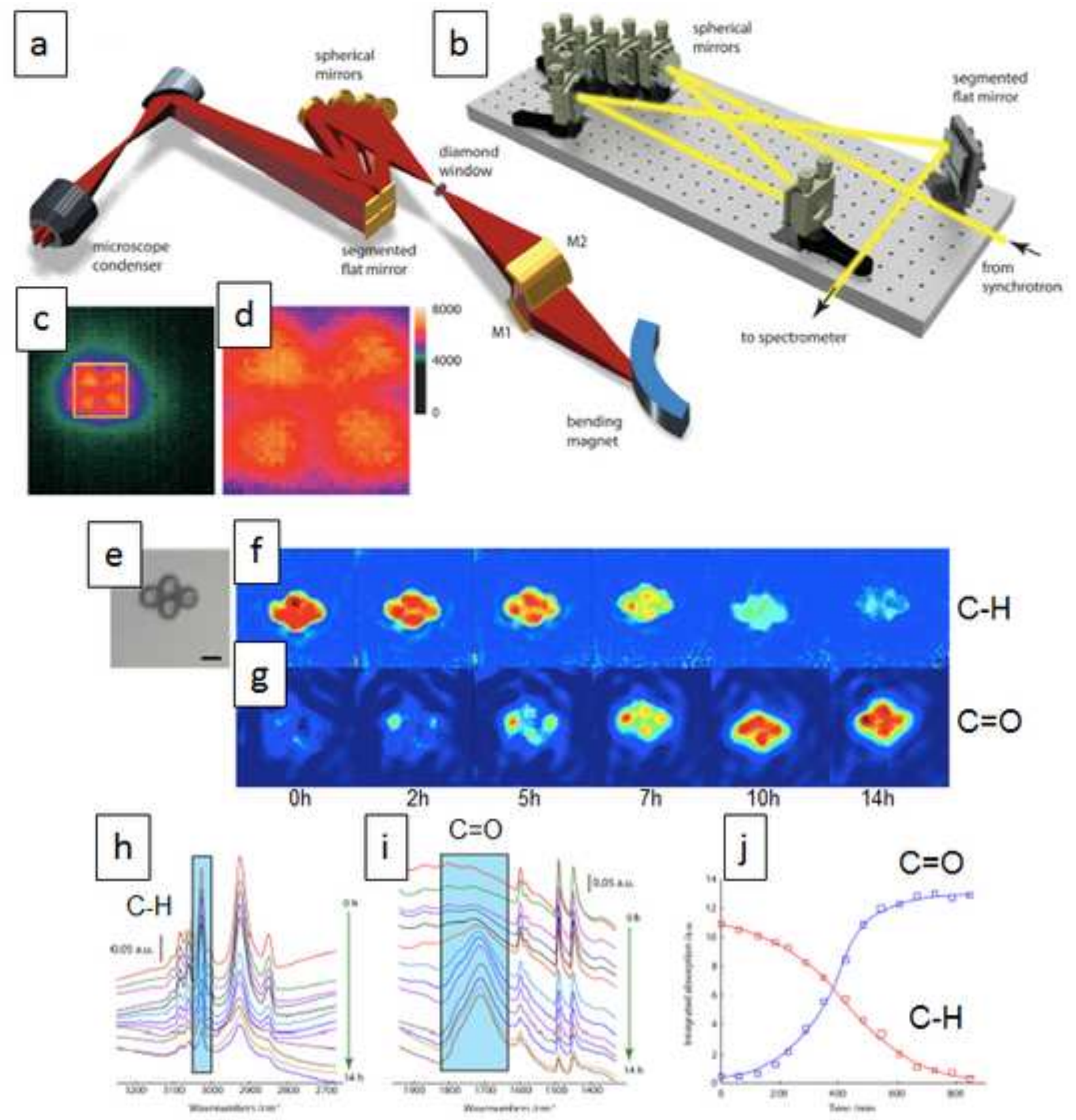

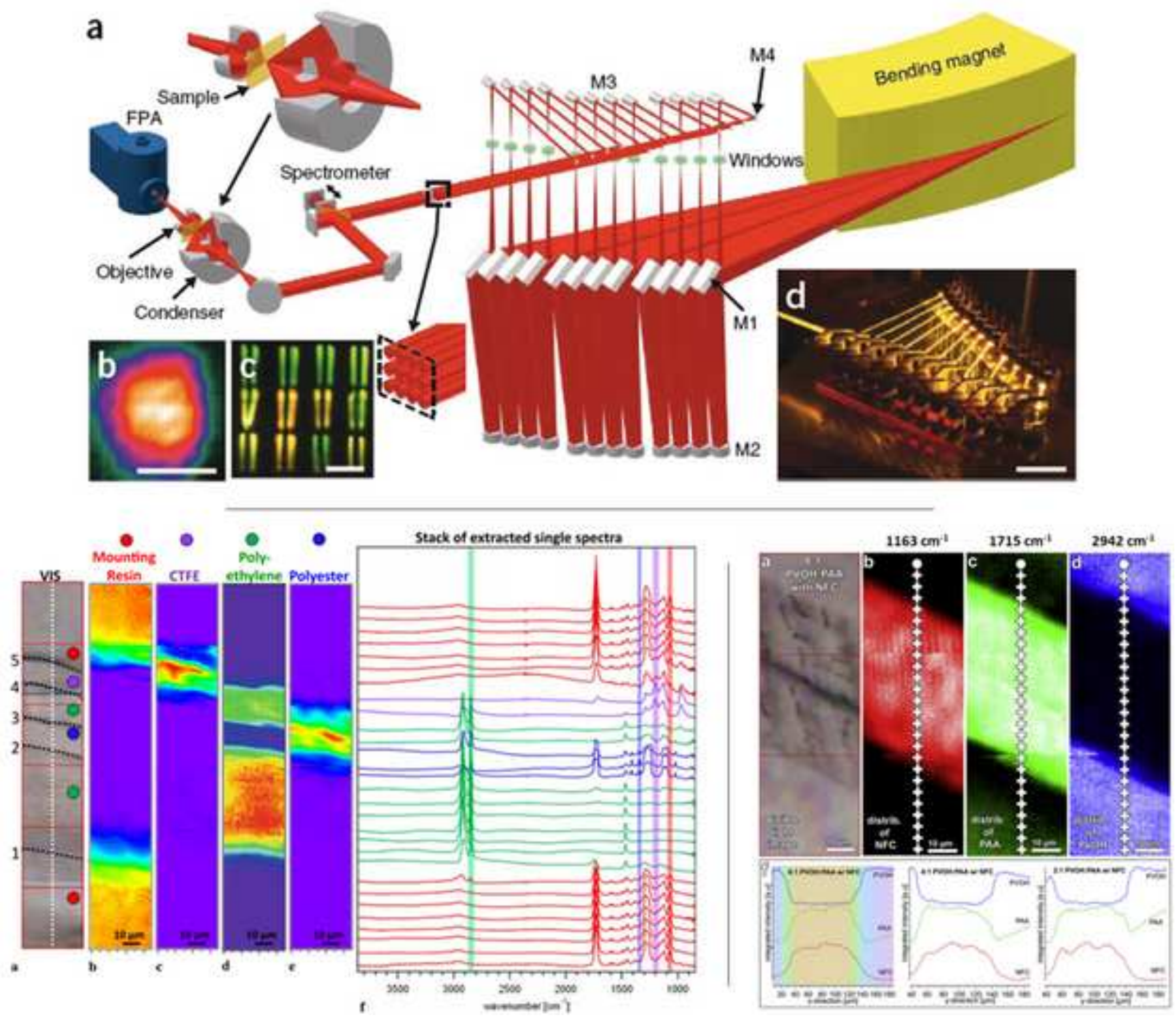
(a)
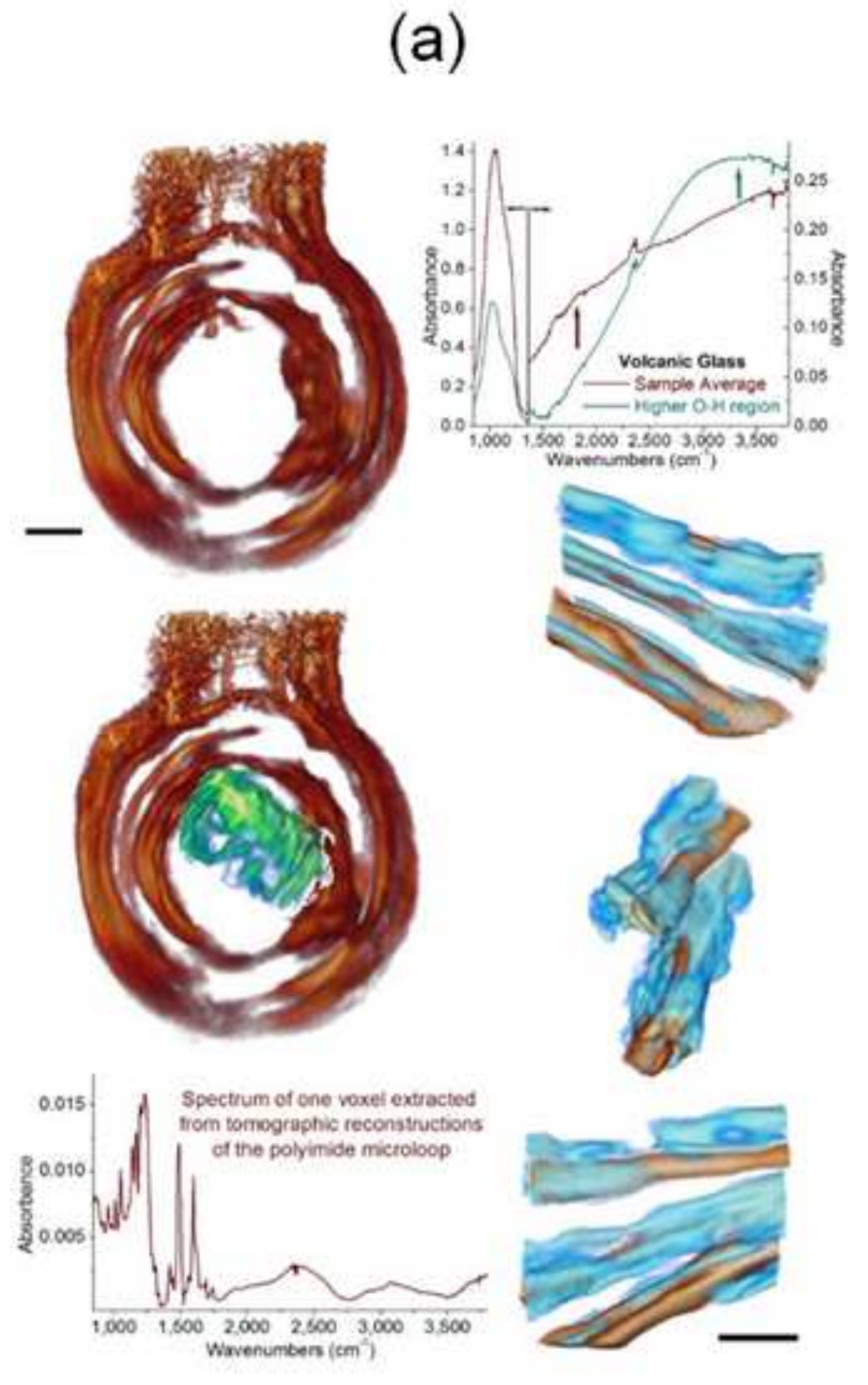

(b)
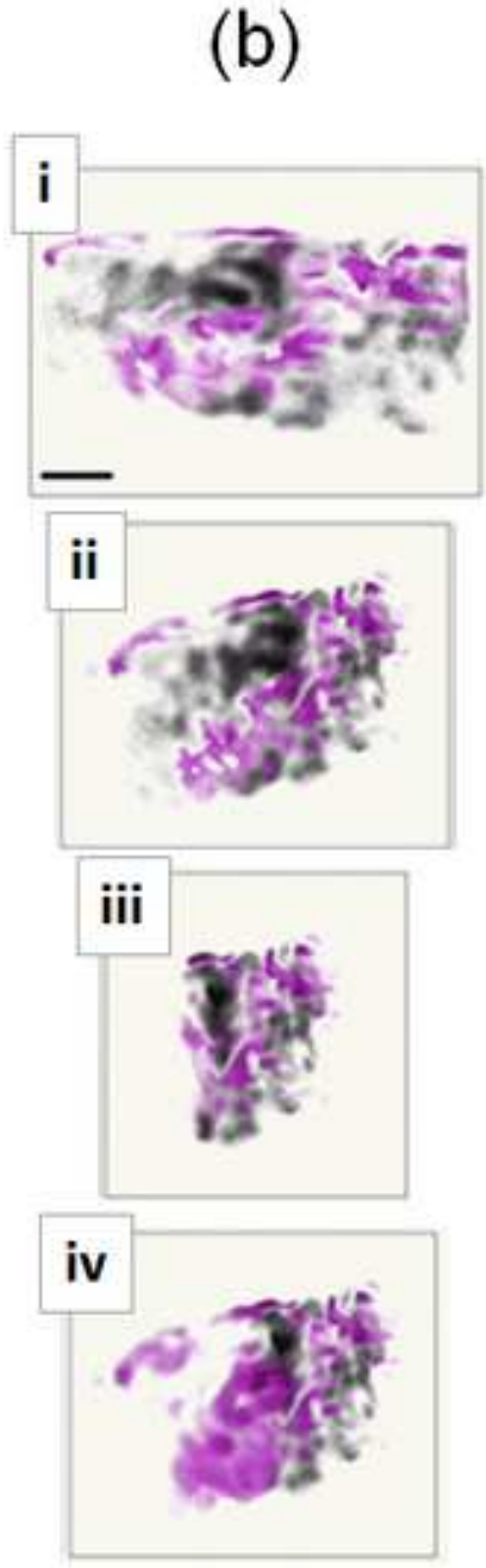


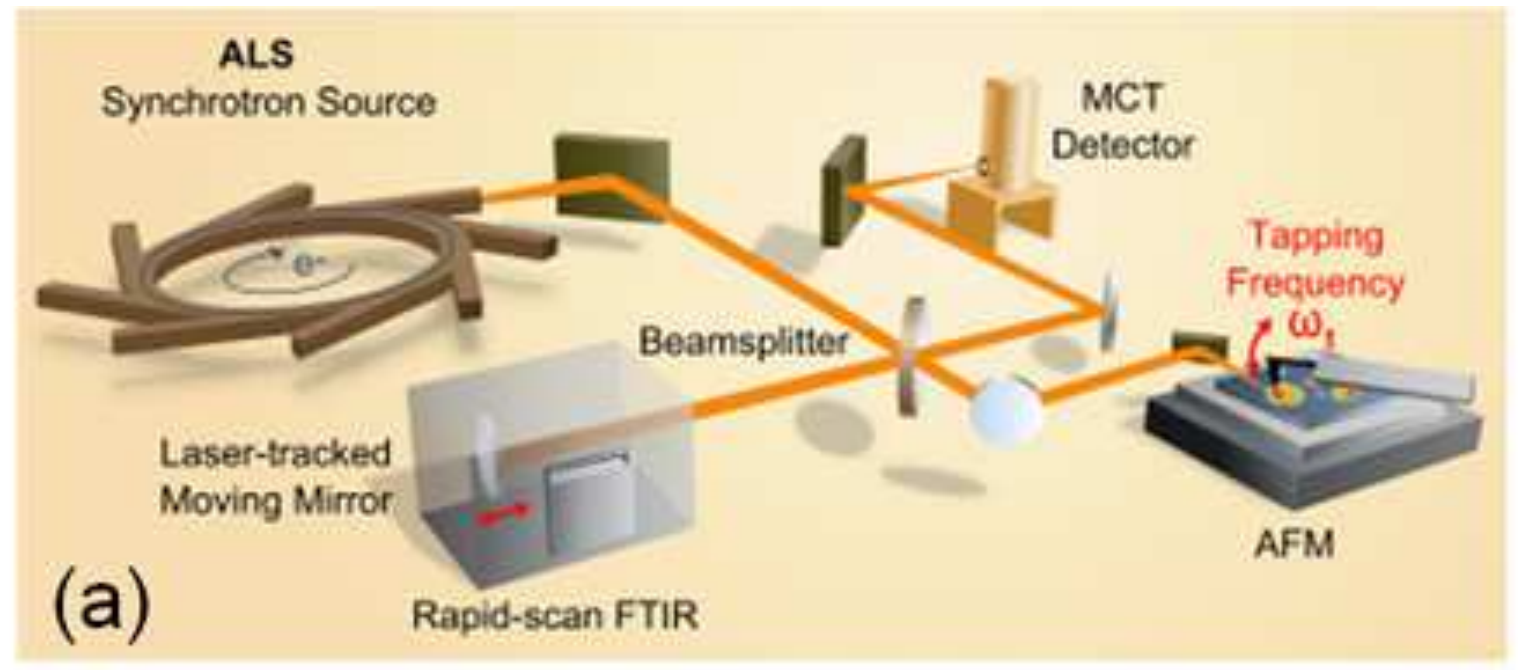

(b)
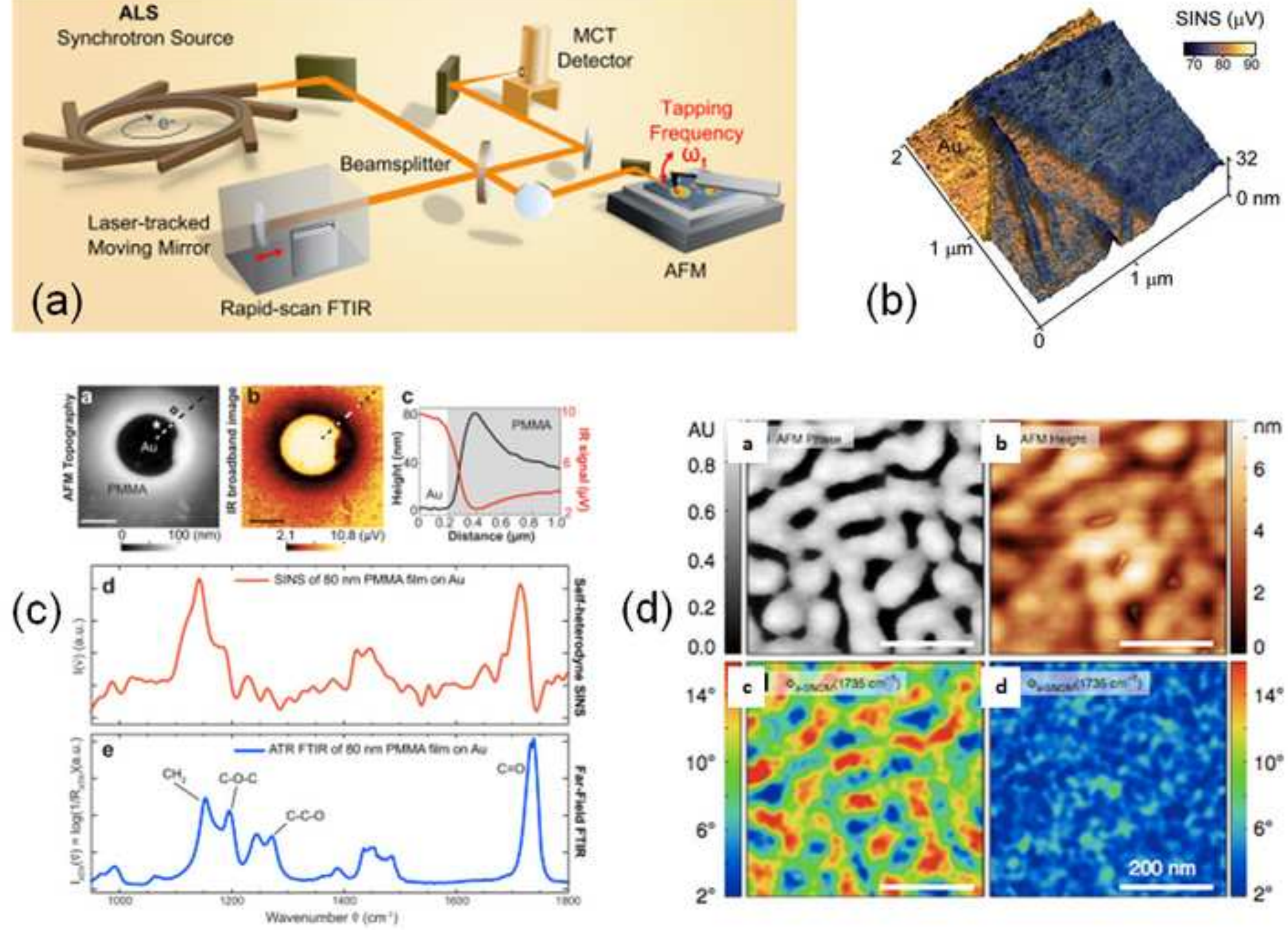
(a)

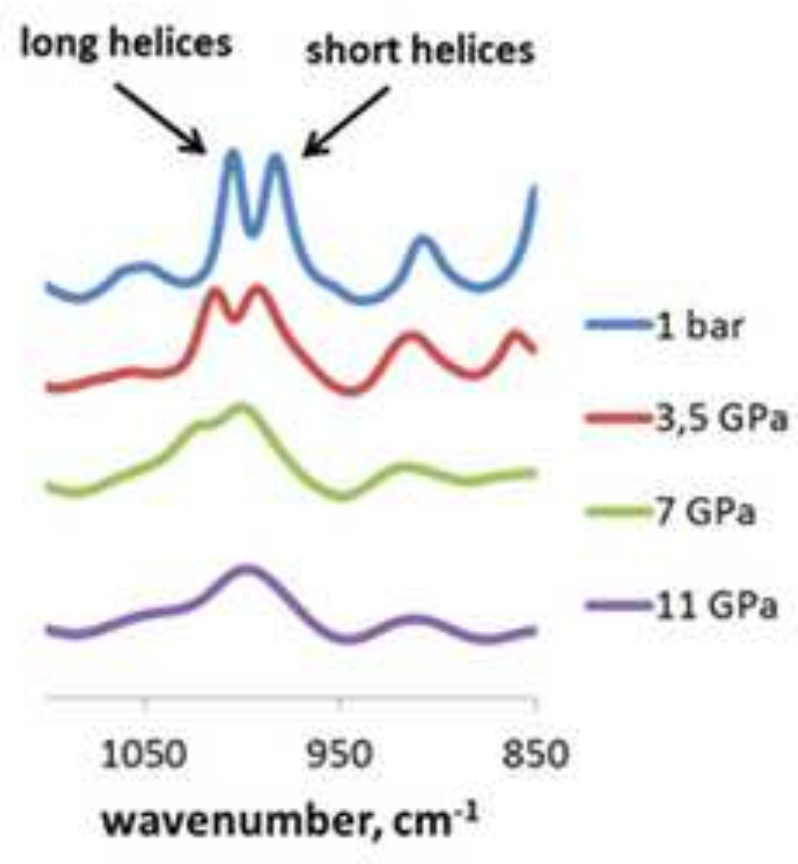

(b)

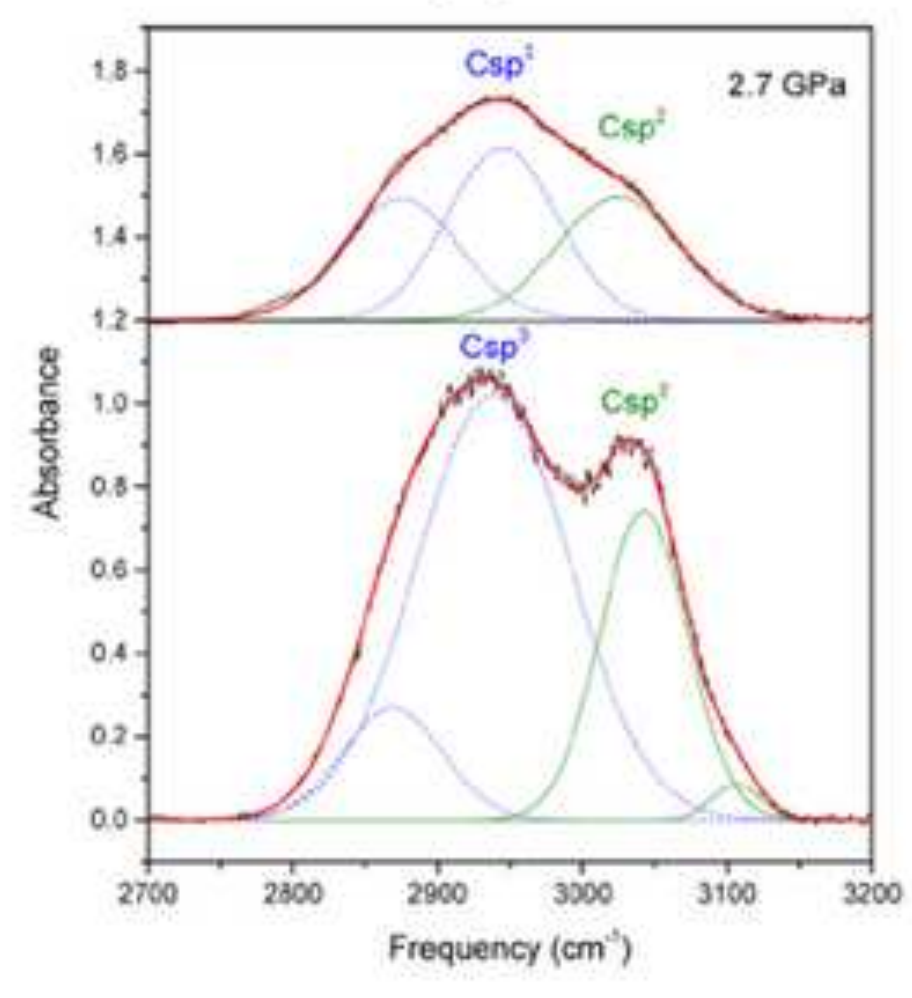

(c)

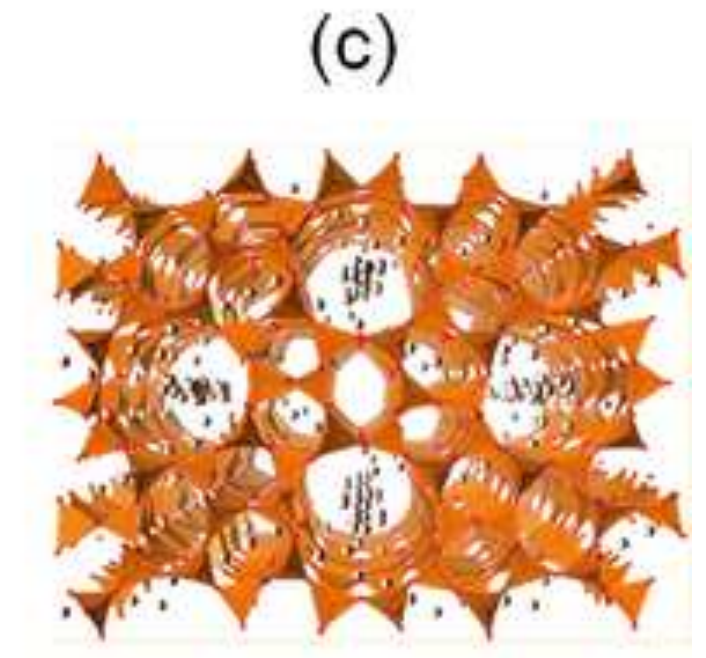

wavenumber,

$$
\text { (2) }
$$



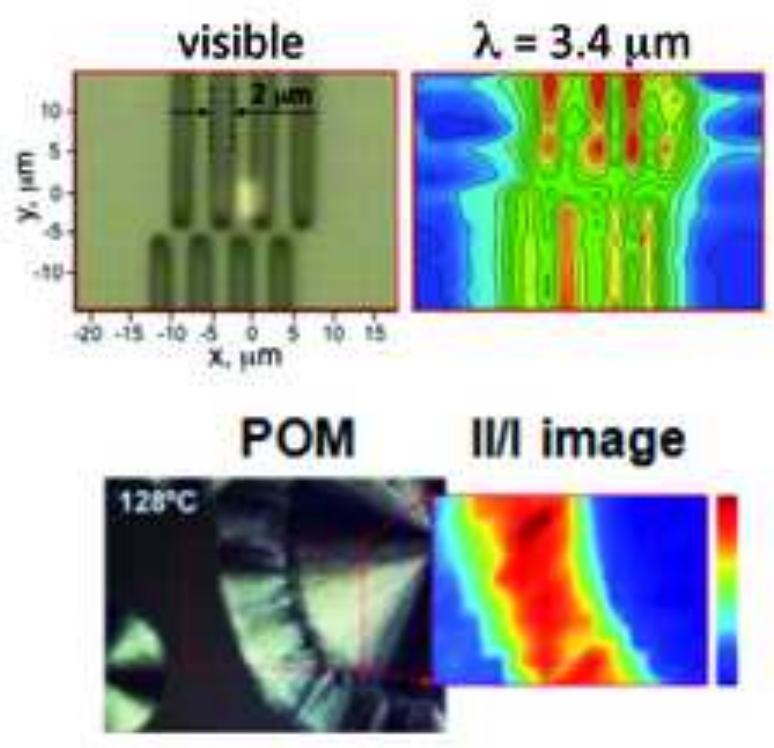

2D

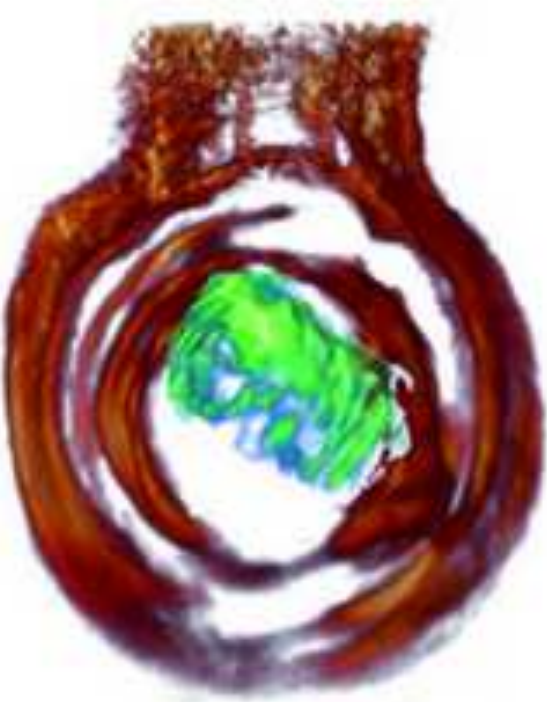

3D

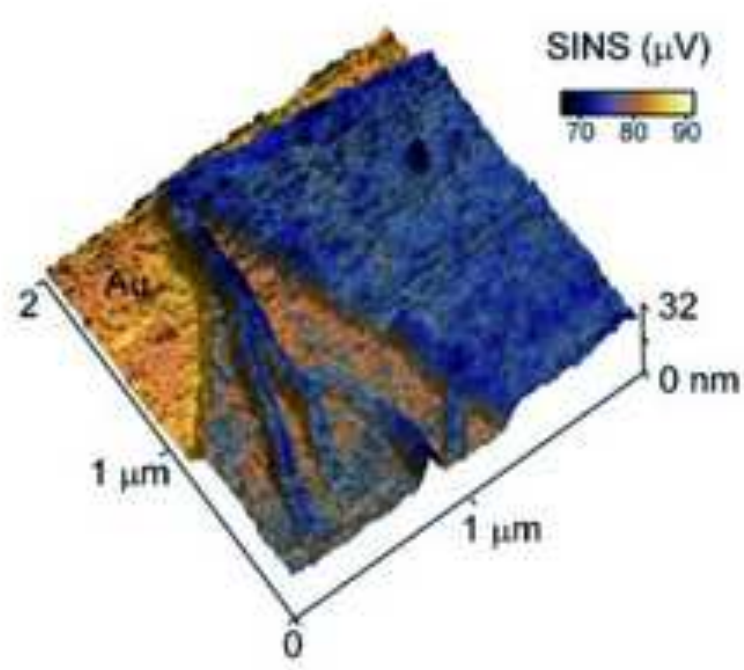

nano-IR 